CRYSTALLOGRAPHIC COMMUNICATIONS

ISSN 2056-9890

Received 14 August 2019

Edited by H. Stoeckli-Evans, University of Neuchâtel, Switzerland

Keywords: crystal structure; aryloxide; ytterbium; mixed valence; arene coordination.

CCDC reference: 1947717

Supporting information: this article has
Accepted 17 August 2019 supporting information at journals.iucr.org/e

\section{Crystal structure of a binuclear mixed-valence ytterbium complex containing a 2-anthracene- substituted phenoxide ligand}

\author{
David J. Berg ${ }^{\mathrm{a} *}$ and Brendan Twamley ${ }^{\mathrm{b}}$
}

a'Department of Chemistry, University of Victoria, PO Box 1700 Stn CSC, Victoria, BC, V8W 2Y2, Canada, and ${ }^{\mathbf{b}}$ School of Chemistry, Trinity College Dublin, University of Dublin, Dublin 2, Ireland. *Correspondence e-mail: djberg@uvic.ca

Reaction of 2-(anthracen-9-yl)phenol (HOPhAn, 1) with divalent $\mathrm{Yb}[\mathrm{N}(\mathrm{Si}$ $\left.\left.\mathrm{Me}_{3}\right)_{2}\right]_{2} \cdot 2 \mathrm{THF}$ in THF-toluene mixtures affords the mixed-valence $\mathrm{Yb}^{\mathrm{II}}-\mathrm{Yb}^{\mathrm{II}}$ dimer $\{[2-($ anthracen-9-yl)phenolato- $\kappa O]$ bis(tetrahydrofuran)ytterbium(III) $\}$ tris $\left[\mu\right.$-2-(anthracen-9-yl)phenolato]- $\kappa^{4} O: O ; \kappa O: 1,2-\eta, \kappa O-\{[2-($ anthracen-9-yl)phenolato- $\kappa O$ ]ytterbium(II) $\}$ toluene trisolvate, $\left[\mathrm{Yb}_{2}\left(\mathrm{C}_{20} \mathrm{H}_{13} \mathrm{O}\right)_{5}\left(\mathrm{C}_{4} \mathrm{H}_{8} \mathrm{O}\right)_{2}\right] \cdot-$ $3 \mathrm{C}_{7} \mathrm{H}_{7}$ or $\left[\mathrm{Yb}^{\mathrm{III}}(\mathrm{THF})_{2}(\mathrm{OPhAn})\right](\mu-\mathrm{OPhAn})_{3}\left[\mathrm{Yb}^{\mathrm{II}}(\mathrm{OPhAn})\right] \cdot 3 \mathrm{C}_{7} \mathrm{H}_{7}$ (2), as the major product. It crystallized as a toluene trisolvate. The $\mathrm{Yb}-\mathrm{O}$ bond lengths in the crystal structure of this dimer clearly identify the $\mathrm{Yb}^{\mathrm{II}}$ and $\mathrm{Yb}^{\mathrm{III}}$ centres. Interestingly, the formally four-coordinate $\mathrm{Yb}^{\mathrm{II}}$ centre shows a close contact with one anthracene $\mathrm{C}-\mathrm{C}$ bond of a bridging $\mathrm{OPhAn}$ ligand, bringing the formal coordination number to five.

\section{Chemical context}

One of the classical methods for introducing ligands into the metal coordination sphere in lanthanide chemistry is by a protonolysis or acid-base reaction that eliminates a suitable protonated ligand from the coordination sphere. The advantage of this method is that it avoids the formation of additional complexes ('ate' salt complexes) often favoured during metathesis reactions (Fig. 1) (Evans, 2000; Volker et al., 2019). However, in the case of divalent ytterbium, the metal is a relatively good reducing agent that can lead to competing redox chemistry with the formation of unexpected trivalent

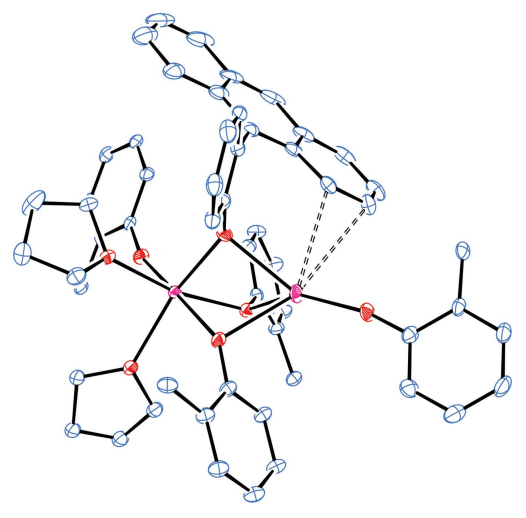

OPEN $\odot$ ACCESS

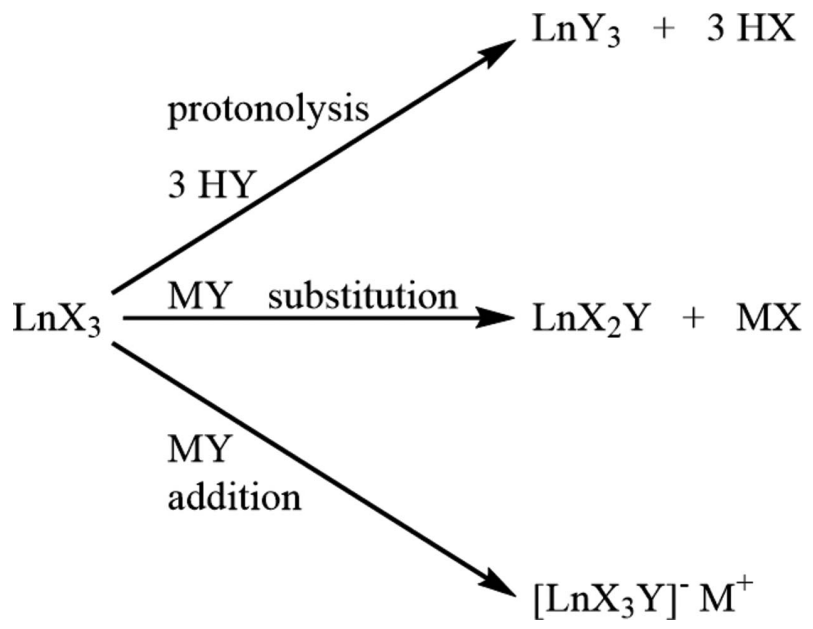

Figure 1

A comparison of the typical outcomes of metathesis with $M Y$ (e.g. $M=$ $\mathrm{Na}^{+}$and $Y=$ anionic ligand) versus protonolysis with $\mathrm{H} Y$ (where $\mathrm{H} Y$ is a stronger acid than $\mathrm{H} X$ ) in lanthanide reactions. 
Table 1

Selected geometric parameters $\left(\AA{ }^{\circ}{ }^{\circ}\right)$.

\begin{tabular}{|c|c|c|c|}
\hline $\mathrm{Yb} 1-\mathrm{Yb} 2$ & 3.2587 (7) & $\mathrm{Yb} 2-\mathrm{O} 1$ & $2.413(3)$ \\
\hline Yb1-O1 & $2.191(3)$ & $\mathrm{Yb} 2-\mathrm{O} 2$ & $2.352(3)$ \\
\hline $\mathrm{Yb} 1-\mathrm{O} 2$ & $2.236(3)$ & $\mathrm{Yb} 2-\mathrm{O} 4$ & $2.165(3)$ \\
\hline $\mathrm{Yb} 1-\mathrm{O} 3$ & 2.077 (3) & $\mathrm{Yb} 2-\mathrm{O} 7$ & $2.416(3)$ \\
\hline $\mathrm{Yb} 1-\mathrm{O} 5$ & $2.306(3)$ & $\mathrm{Yb} 2-\mathrm{C} 18 D$ & $3.190(3)$ \\
\hline $\mathrm{Yb} 1-\mathrm{O} 6 A$ & $2.309(6)$ & $\mathrm{Yb} 2-\mathrm{C} 19 D$ & $2.970(3)$ \\
\hline Yb1-O7 & $2.229(3)$ & & \\
\hline $\mathrm{O} 1-\mathrm{Yb} 1-\mathrm{O} 2$ & $82.10(11)$ & $\mathrm{O} 7-\mathrm{Yb} 1-\mathrm{O} 5$ & $103.45(11)$ \\
\hline $\mathrm{O} 1-\mathrm{Yb} 1-\mathrm{O} 5$ & $86.34(12)$ & $\mathrm{O} 7-\mathrm{Yb} 1-\mathrm{O} 6 A$ & $82.0(3)$ \\
\hline $\mathrm{O} 1-\mathrm{Yb} 1-\mathrm{O} 7$ & $77.59(12)$ & $\mathrm{O} 1-\mathrm{Yb} 2-\mathrm{O} 7$ & $69.99(10)$ \\
\hline $\mathrm{O} 1-\mathrm{Yb} 1-\mathrm{O} 6 A$ & $158.2(3)$ & $\mathrm{O} 2-\mathrm{Yb} 2-\mathrm{O} 1$ & $75.17(10)$ \\
\hline $\mathrm{O} 2-\mathrm{Yb} 1-\mathrm{O} 5$ & $168.04(11)$ & $\mathrm{O} 2-\mathrm{Yb} 2-\mathrm{O} 7$ & $71.36(11)$ \\
\hline $\mathrm{O} 2-\mathrm{Yb} 1-\mathrm{O} 6 A$ & $100.6(5)$ & $\mathrm{O} 4-\mathrm{Yb} 2-\mathrm{O} 1$ & $137.75(12)$ \\
\hline $\mathrm{O} 3-\mathrm{Yb} 1-\mathrm{O} 1$ & $106.85(12)$ & $\mathrm{O} 4-\mathrm{Yb} 2-\mathrm{O} 2$ & $147.05(12)$ \\
\hline $\mathrm{O} 3-\mathrm{Yb} 1-\mathrm{O} 2$ & $94.92(12)$ & $\mathrm{O} 4-\mathrm{Yb} 2-\mathrm{O} 7$ & $115.61(11)$ \\
\hline $\mathrm{O} 3-\mathrm{Yb} 1-\mathrm{O} 5$ & $85.50(12)$ & $\mathrm{Yb} 1-\mathrm{O} 1-\mathrm{Yb} 2$ & $89.98(11)$ \\
\hline $\mathrm{O} 3-\mathrm{Yb} 1-\mathrm{O} 7$ & $170.39(12)$ & $\mathrm{Yb} 1-\mathrm{O} 2-\mathrm{Yb} 2$ & 90.47 (11) \\
\hline $\mathrm{O} 3-\mathrm{Yb} 1-\mathrm{O} 6 A$ & $94.5(3)$ & $\mathrm{Yb} 1-\mathrm{O} 7-\mathrm{Yb} 2$ & $88.99(11)$ \\
\hline $\mathrm{O} 5-\mathrm{Yb} 1-\mathrm{O} 6 A$ & $91.2(5)$ & $\mathrm{C} 1 A-\mathrm{O} 3-\mathrm{Yb} 1$ & $162.0(3)$ \\
\hline $\mathrm{O} 7-\mathrm{Yb} 1-\mathrm{O} 2$ & 77.07 (11) & $\mathrm{C} 1 C-\mathrm{O} 4-\mathrm{Yb} 2$ & $166.3(3)$ \\
\hline
\end{tabular}

ytterbium products (Kagan \& Namy, 1984). We have observed that these redox reactions become more problematic the more acidic the incoming 'acid' is. In the case of alcohols and phenols, reduction of the $\mathrm{O}-\mathrm{H}$ bond is sometimes observed but there are also instances where the product remains divalent (Delbridge et al., 2007; Binda et al., 2008). In the case of

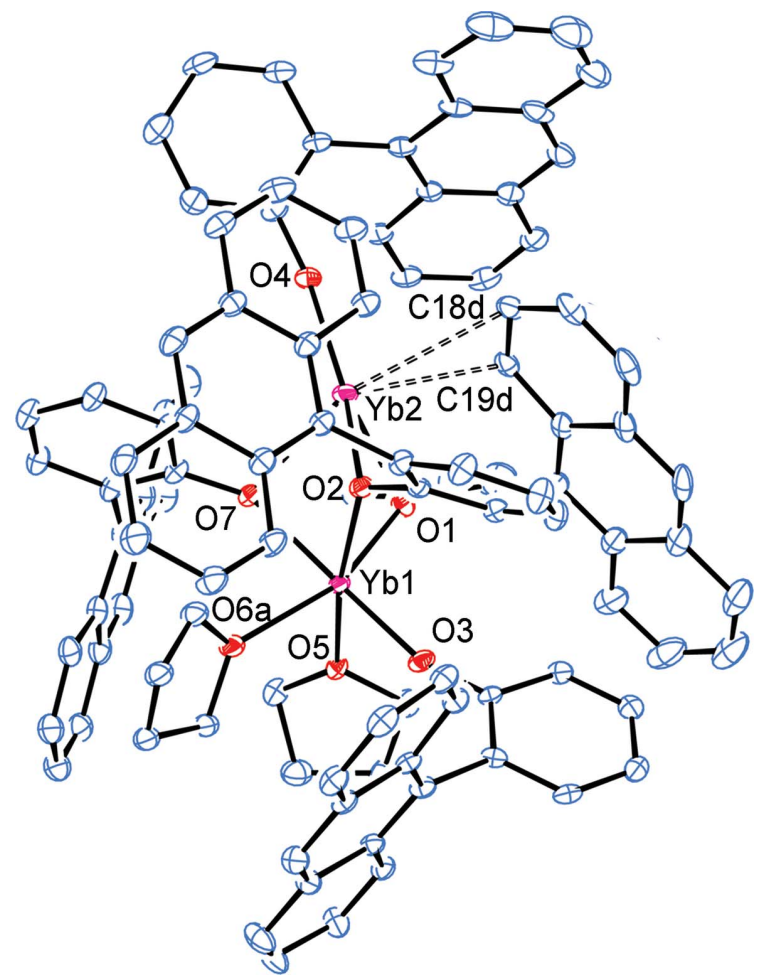

Figure 2

The molecular structure of complex $\mathbf{2}$, with partial atom labelling and displacement ellipsoids drawn at the $30 \%$ probability level (as also for Figs. 3 and 4). For clarity, the toluene molecules of solvation and $\mathrm{H}$ atoms have been omitted in this and other figures. Only the major-disorder partner is shown for THF molecule $\mathrm{O} 6 a / \mathrm{C} 21 a-\mathrm{C} 24 a$ and the OPhAn ligand $\mathrm{C} 1 e-\mathrm{C} 20 e$. 2-(anthracen-9-yl)phenol (HOPhAn) discussed here, partial $\mathrm{O}-\mathrm{H}$ reduction is observed, resulting in a mixed-valence $\mathrm{Yb}^{\mathrm{II}}-\mathrm{Yb}^{\mathrm{III}}$ dimer featuring bridging aryloxides.

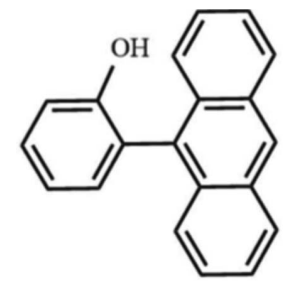

HOPhAn, 1

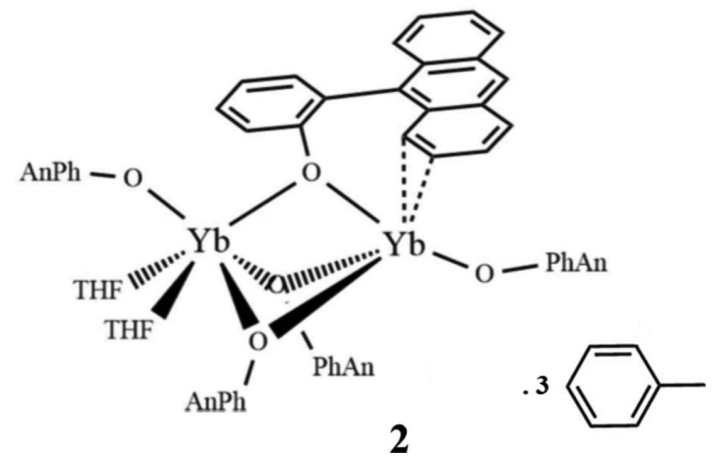

\section{Structural commentary}

The structure of complex 2 (without the three toluenes of solvation) is shown in Fig. 2. The inner coordination environment is depicted in Figs. 3 and 4. In Fig. 3, the anthracene groups have been removed for clarity, except for the 9-anthracene C atoms. In Fig. 4, the structure has been further

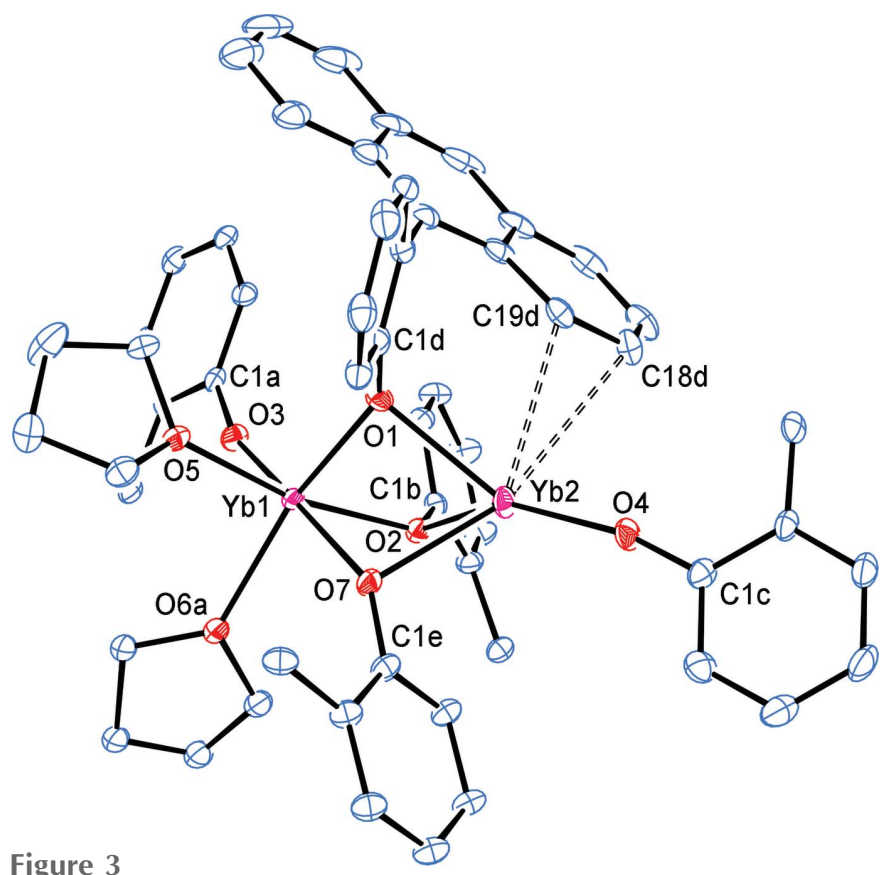

A simplified view of complex 2 , removing all anthracene $\mathrm{C}$ atoms except those attached to the phenyl rings or part of the weakly interacting anthracene unit. 


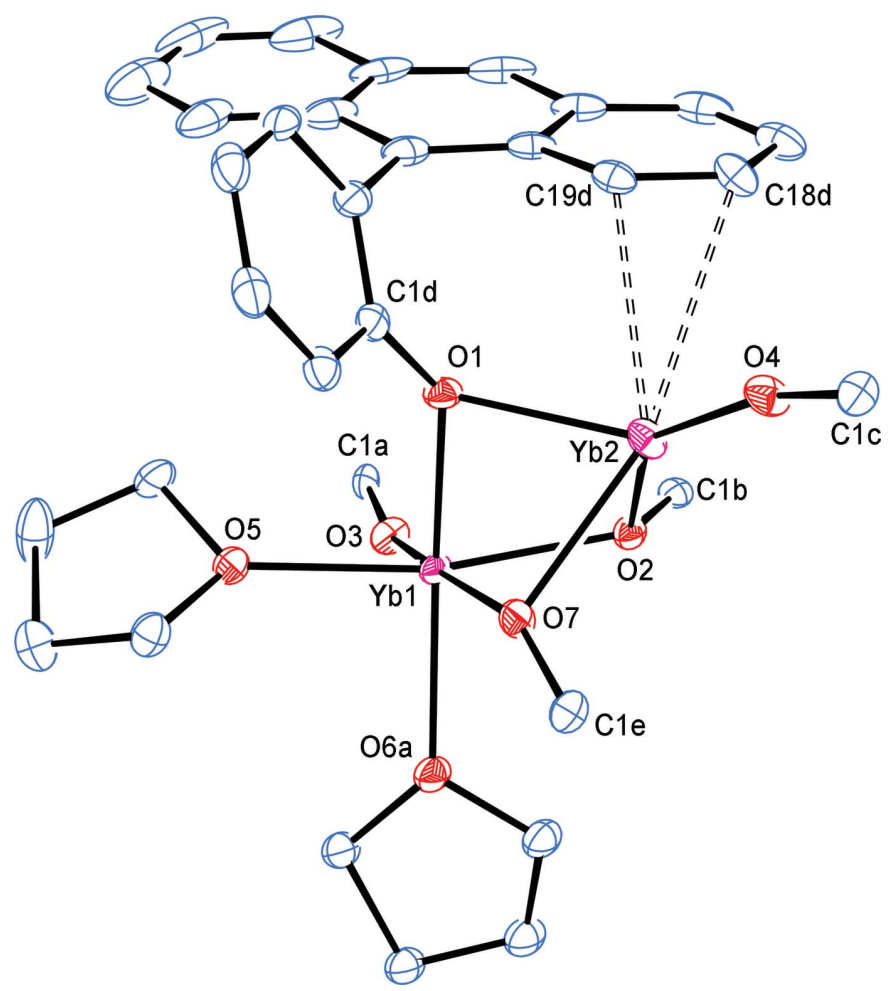

Figure 4

A view of the inner coordination sphere of complex 2. Only the phenoxide $\mathrm{C}$ atom attached to an $\mathrm{O}$ atom is shown for all ligands, except for that of the coordinated anthracene group.

simplified by removing all of the $\mathrm{PhAn} \mathrm{C}$ atoms except that directly attached to the phenoxide $\mathrm{O}$ atom. In both Figs. 3 and 4, all the $\mathrm{C}$ atoms of the PhAn ligand that show close contacts with $\mathrm{Yb} 2$ through atoms $\mathrm{C} 18 d$ and $\mathrm{C} 19 d$ are depicted.

The two $\mathrm{Yb}$ centres in 2 are connected [Yb1 $\cdots \mathrm{Yb} 2=$ 3.2587 (7) $\AA$; Table 1] by three bridging OPhAn aryloxides and each $\mathrm{Yb}$ atom is also bound to two terminal aryloxides. Atom $\mathrm{Yb} 1$ is further coordinated by two THF ligands, bringing the coordination number to 6 , while $\mathrm{Yb} 2$ shows the aforementioned close contacts with $\mathrm{C} 18 d$ and $\mathrm{C} 19 d$ for a coordination number of 5 (taking the $\mathrm{C} 18 d-\mathrm{C} 19 d$ bond as occupying the fifth coordination site). Based on the stoichiometry, 2 is expected to be a $\mathrm{Yb}^{\mathrm{II}} / \mathrm{Yb}^{\mathrm{III}}$ mixed-valence complex. Selected bond lengths and angles and close contacts between $\mathrm{Yb} 2$ and the $\mathrm{C}$ atoms of one anthracene group are listed in Table 1. The substantially shorter terminal and bridging Yb1OPhAn distances [terminal: 2.077 (3) Å; bridging: 2.191 (3)$2.236(3) \AA$ c compared to Yb2-OPhAn [terminal:

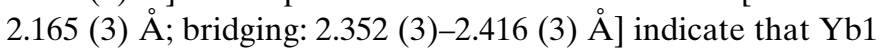
is trivalent, while $\mathrm{Yb} 2$ is divalent.

For ytterbium, the Cambridge Structural Database (CSD, Version 5.38, last update November 2018; Groom et al., 2016) lists 10 bridging and 88 terminal structures containing nonchelating aryloxides. These structures represent both $\mathrm{Yb}^{\mathrm{II}}$ and $\mathrm{Yb}^{\mathrm{III}}$ oxidation states but there is only one structure containing a bridging aryloxide that contains both $\mathrm{Yb}^{\mathrm{II}}$ and $\mathrm{Yb}^{\mathrm{III}}$ in a mixed-valence complex (Deacon et al., 2009). The supporting information (CSD search S1) lists the CSD refcodes and references of structures containing Yb terminal and bridging nonchelating aryloxides used in the structural discussion below.

The terminal and bridging distances for Yb1 compare well with other six-coordinate trivalent $\mathrm{Yb}$ aryloxides (terminal: 2.023-2.258, median 2.077 ̊; bridging: 2.215-2.246, median $2.240 \AA$ ).

The terminal and bridging distances for $\mathrm{Yb} 2$ also agree fairly well with other five-coordinate divalent $\mathrm{Yb}$ aryloxides (terminal: 2.168-2.233, median 2.215 А; bridging: 2.245-2.356, median $2.309 \AA$ ).

The terminal $\mathrm{Yb} 2-\mathrm{OPhAn}$ distance is at the short end of the observed range, while the bridging distance is somewhat longer than expected based on literature comparisons (see S1 in the supporting information).

The Yb1-O(THF) distances [2.306 (3) and 2.309 (6) $\AA$ ] are typical of six-coordinate $\mathrm{Yb}^{\mathrm{III}}-\mathrm{O}(\mathrm{THF})$ bond lengths (2.251-2.509 $\AA$; median 2.342 $\AA$ ) and indicate that the $\mathrm{Yb}$ centre is not overly crowded (see CSD search S1 in the supporting information). The most noteworthy feature in the structure of $\mathbf{2}$ is the presence of a close contact between two anthracene $\mathrm{C}$ atoms of one bridging $\mathrm{OPhAn}$ ligand and $\mathrm{Yb} 2$ $[\mathrm{Yb} 2-\mathrm{C} 18 \mathrm{~d}=3.190$ (3) $\AA$ and $\mathrm{Yb} 2-\mathrm{C} 19 \mathrm{~d}=2.970$ (3) $\AA$ ] This is well within the wide range recognized for weak $\mathrm{Yb}^{\mathrm{II}}$ arene interactions (2.795-3.651 $\mathrm{A}$, median $2.98 \AA$ ) after correction to five-coordination.

It appears that the $\mathrm{Yb}^{\mathrm{II}}$ centre prefers to satisfy its electron deficiency by coordination of an anthracene $\pi$-bond (or the anthracene $\mathrm{C}-\mathrm{H}$ bonds) rather than coordinating a further molecule of THF.

\section{Supramolecular features}

The only noteworthy interactions observable in the crystal structure of complex 2 are $\mathrm{C}-\mathrm{H} \cdots \pi$ interactions, both intraand intermolecular (see Table S2 in the supporting informa-

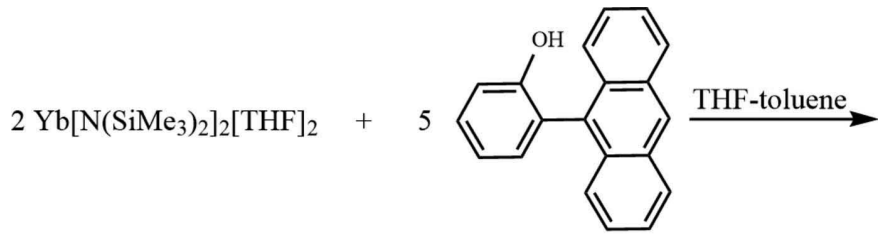

HOPhAn, 1

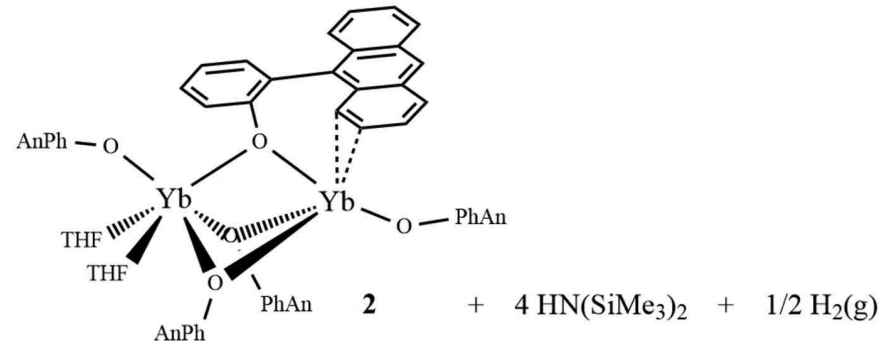

Figure 5

The reaction scheme for the synthesis of complex 2 . 
Table 2

Experimental details.

\begin{tabular}{|c|c|}
\hline \multicolumn{2}{|l|}{ Crystal data } \\
\hline Chemical formula & {$\left[\mathrm{Yb}_{2}\left(\mathrm{C}_{20} \mathrm{H}_{13} \mathrm{O}\right)_{5}\left(\mathrm{C}_{4} \mathrm{H}_{8} \mathrm{O}\right)_{2}\right] \cdot 3 \mathrm{C}_{7} \mathrm{H}_{7}$} \\
\hline$M_{\mathrm{r}}$ & 2113.20 \\
\hline Crystal system, space group & Monoclinic, $P 2_{1} / c$ \\
\hline Temperature $(\mathrm{K})$ & 83 \\
\hline$a, b, c(\AA)$ & $\begin{array}{l}14.4612(7), 20.8473(10) \\
32.0864(15)\end{array}$ \\
\hline$\beta\left({ }^{\circ}\right)$ & $95.508(1)$ \\
\hline$V\left(\AA^{3}\right)$ & $9628.6(8)$ \\
\hline$Z$ & 4 \\
\hline Radiation type & Мо $K \alpha$ \\
\hline$\mu\left(\mathrm{mm}^{-1}\right)$ & 1.99 \\
\hline Crystal size $(\mathrm{mm})$ & $0.4 \times 0.37 \times 0.1$ \\
\hline \multicolumn{2}{|l|}{ Data collection } \\
\hline Diffractometer & $\begin{array}{l}\text { Bruker SMART CCD area } \\
\text { detector }\end{array}$ \\
\hline Absorption correction & $\begin{array}{l}\text { Multi-scan ( } S A D A B S ; \text { Bruker, } \\
\text { 2002) }\end{array}$ \\
\hline$T_{\min }, T_{\max }$ & $0.729,0.826$ \\
\hline $\begin{array}{l}\text { No. of measured, independent and } \\
\text { observed }[I>2 \sigma(I)] \text { reflections }\end{array}$ & $109364,22107,15925$ \\
\hline$R_{\text {int }}$ & 0.067 \\
\hline$(\sin \theta / \lambda)_{\max }\left(\AA^{-1}\right)$ & 0.650 \\
\hline \multicolumn{2}{|l|}{ Refinement } \\
\hline$R\left[F^{2}>2 \sigma\left(F^{2}\right)\right], w R\left(F^{2}\right), S$ & $0.048,0.115,1.03$ \\
\hline No. of reflections & 22107 \\
\hline No. of parameters & 1480 \\
\hline No. of restraints & 1509 \\
\hline $\mathrm{H}$-atom treatment & H-atom parameters constrained \\
\hline$\Delta \rho_{\max }, \Delta \rho_{\min }\left(\mathrm{e} \AA^{-3}\right)$ & $2.20,-0.93$ \\
\hline
\end{tabular}

Computer programs: SMART (Bruker, 2002), SAINT (Bruker, 2002), SHELXS (Sheldrick, 2008), SHELXL (Sheldrick, 2015) and OLEX2 (Dolomanov et al., 2009).

tion). Interstitial space is filled with three toluene molecules of solvation (all disordered over two positions; see §5, Refinement).

\section{Synthesis and crystallization}

\subsection{Materials and instrumentation}

All solvents were purchased from Sigma-Aldrich Chemicals and dried by distillation from sodium under nitrogen. The 2-(anthracen-9-yl)phenol was purified by recrystallization from hot toluene, while $\mathrm{Yb}\left[\mathrm{N}(\mathrm{SiMe})_{3}\right]_{2}(\mathrm{THF})_{2}$ was prepared by analogy to the procedure of Hitchcock et al. (2002) using $\mathrm{NaN}\left(\mathrm{SiMe}_{3}\right)_{2}$ and $\mathrm{YbI}_{2}(\mathrm{THF})_{x}$, and recrystallized from hot hexane. NMR spectra were recorded on a Bruker AV III $300 \mathrm{MHz}$ Spectrometer in sealable Teflon-valved tubes and were referenced to residual solvent resonances. The line widths at half maximum $\left(v_{1 / 2}\right.$ in $\left.\mathrm{Hz}\right)$ were measured for all paramagnetic resonances and are reported below. Elemental analyses were performed by Canadian Microanalytical Ltd.

\subsection{Synthesis of complex 2}

The reaction scheme for the synthesis of complex $\mathbf{2}$ is illustrated in Fig. 5. A solution of 2-(anthracen-9-yl)phenol $(0.100 \mathrm{~g}, 0.370 \mathrm{mmol})$ in THF $(10 \mathrm{ml})$ was prepared in a glovebox and added by Pasteur pipette to a vigorously stirred solution of $\mathrm{Yb}\left[\mathrm{N}(\mathrm{SiMe})_{3}\right]_{2}(\mathrm{THF})_{2}(0.078 \mathrm{~g}, 0.12 \mathrm{mmol})$ in toluene $(10 \mathrm{ml})$. The deep-orange solution darkened to red on stirring overnight. The solution was filtered through Celite on a sintered glass frit and the filtrate was evaporated to dryness under reduced pressure. The red solid was recrystallized from a mixture of toluene and hexane at $143 \mathrm{~K}$, yielding deeporange crystals (yield $0.079 \mathrm{~g}, 62 \%) .{ }^{1} \mathrm{H}$ NMR $\left(\mathrm{C}_{6} \mathrm{D}_{6}\right.$, $300 \mathrm{MHz}, 296 \mathrm{~K}): \delta 88.4\left(6 \mathrm{H}, v_{1 / 2}=700 \mathrm{~Hz}\right), 49.4(3 \mathrm{H}$, overlaps next resonance), $47.9\left(6 \mathrm{H}, v_{1 / 2}=350 \mathrm{~Hz}\right.$, overlaps previous resonance), $12.86\left(2 \mathrm{H}, v_{1 / 2}=9 \mathrm{~Hz}\right), 11.70\left(4 \mathrm{H}, v_{1 / 2}=12 \mathrm{~Hz}\right)$, $10.93\left(4 \mathrm{H}, v_{1 / 2}=12 \mathrm{~Hz}\right), 10.00\left(4 \mathrm{H}, v_{1 / 2}=25 \mathrm{~Hz}\right), 9.30(4 \mathrm{H}$, $\left.v_{1 / 2}=70 \mathrm{~Hz}\right), 1.26(2 \mathrm{H}, t), 0.96(2 \mathrm{H}, t),-2.77\left(3 \mathrm{H}, v_{1 / 2}=\right.$ $100 \mathrm{~Hz}),-3.89\left(2 \mathrm{H}, v_{1 / 2}=14 \mathrm{~Hz}\right),-5.38\left(2 \mathrm{H}, v_{1 / 2}=20 \mathrm{~Hz}\right)$, $-11.2\left(6 \mathrm{H}, v_{1 / 2} \sim 150 \mathrm{~Hz}\right.$, overlaps next resonance), -11.4 $\left(6 \mathrm{H}, v_{1 / 2} \sim 300 \mathrm{~Hz}\right.$, overlaps previous resonance), $-16.0(3 \mathrm{H}$, $\left.v_{1 / 2}=140 \mathrm{~Hz}\right),-24.4\left(3 \mathrm{H}, v_{1 / 2}=800 \mathrm{~Hz}\right),-77.2\left(3 \mathrm{H}, v_{1 / 2}=\right.$ $600 \mathrm{~Hz}$ ). Analysis calculated for $\mathrm{C}_{129} \mathrm{H}_{105} \mathrm{O}_{7} \mathrm{Yb}_{2}$ (\%): C 73.30, H 5.01; found: C 72.55, H 4.87 .

\section{Refinement}

Crystal data, data collection and structure refinement details are summarized in Table 2. The $\mathrm{C}$-bound $\mathrm{H}$ atoms were included in calculated positions and treated as riding atoms: $\mathrm{C}-\mathrm{H}=0.95-0.99 \AA$, with $U_{\text {iso }}(\mathrm{H})=1.5 U_{\text {eq }}(\mathrm{C})$ for methyl $\mathrm{H}$ atoms and $1.2 U_{\text {eq }}(\mathrm{C})$ for other $\mathrm{H}$ atoms.

One bridging ligand, $\mathrm{O} 7 / \mathrm{C} 1 e-\mathrm{C} 20 e: \mathrm{O} 7 / \mathrm{C} 1 f-\mathrm{C} 20 f$, was disordered and modelled in two positions, with an occupancy ratio of 0.533 (3):0.467 (3), using rigid groups and restraints. A metal-coordinated THF molecule, O6a/C21a-C24a:O6b/ $\mathrm{C} 21 b-\mathrm{C} 24 b$, is also disordered and was modelled in two positions, with an occupancy ratio of 0.542 (9):0.458 (9), using restraints. Three toluene solvent molecules are also present and are all disordered over two positions; they were modelled with rigid groups and restraints; the $\mathrm{C} 29$ and $\mathrm{C} 43$ toluene molecules were modelled at 50\% occupancy each and the C36 toluene molecule was modelled with an occupancy ratio of 0.682 (9):0.318 (9).

The largest peak in the final difference electron density synthesis is near atom $\mathrm{Yb} 1$ and the largest hole is near atom $\mathrm{C} 19 d$; the r.m.s. deviation is $0.11 \mathrm{e} \AA^{-3}$.

\section{Acknowledgements}

DJB wishes to acknowledge the preliminary investigations of Karen Button that led to this work. Funding for this research was provided by the Natural Sciences and Engineering Research Council of Canada.

\section{References}

Binda, P. L., Delbridge, E. E., Dugah, D. T., Skelton, B. W. \& White, A. H. (2008). Z. Anorg. Allg. Chem. 634, 325-334.

Bruker (2002). APEX2, SAINT and SADABS. Bruker AXS Inc., Madison, Wisconsin, USA.

Deacon, G. B., Junk, P. C. \& Moxey, G. J. (2009). Chem. Asian J. 4, 1717-1728.

Delbridge, E. E., Dugah, D. T., Nelson, C. R., Skelton, B. W. \& White, A. H. (2007). Dalton Trans. pp. 143-153. 
Dolomanov, O. V., Bourhis, L. J., Gildea, R. J., Howard, J. A. K. \& Puschmann, H. (2009). J. Appl. Cryst. 42, 339-341.

Evans, W. J. (2000). Coord. Chem. Rev. 206-207, 263-283.

Groom, C. R., Bruno, I. J., Lightfoot, M. P. \& Ward, S. C. (2016). Acta Cryst. B72, 171-179.

Hitchcock, P. B., Khvostok, A. V., Lappert, M. F. \& Protchenko, A. V. (2002). J. Organomet. Chem. 647, 198-204.
Kagan, H. B. \& Namy, J. L. (1984). Handb. Chem. Phys. Rare Earths, 6, 52 .

Sheldrick, G. M. (2008). Acta Cryst. A64, 112-122.

Sheldrick, G. M. (2015). Acta Cryst. C71, 3-8.

Volker, L., Liebing, P., Engelhardt, F., Stein, F., Kuehling, M., Schroeder, L. \& Edelmann, F. T. (2019). J. Coord. Chem. 72, 1-34. 


\section{supporting information}

Acta Cryst. (2019). E75, 1367-1371 [https://doi.org/10.1107/S205698901901154X]

\section{Crystal structure of a binuclear mixed-valence ytterbium complex containing a}

\section{2-anthracene-substituted phenoxide ligand}

\section{David J. Berg and Brendan Twamley}

Computing details

Data collection: SMART (Bruker, 2002); cell refinement: SAINT (Bruker, 2002); data reduction: SAINT (Bruker, 2002); program(s) used to solve structure: SHELXS (Sheldrick, 2008); program(s) used to refine structure: SHELXL (Sheldrick, 2015); molecular graphics: OLEX2 (Dolomanov et al., 2009); software used to prepare material for publication: OLEX2 (Dolomanov et al., 2009).

\{[2-(Anthracen-9-yl) phenolato- $\kappa O]$ bis(tetrahydrofuran)ytterbium(III)\}-tris $[\mu$-2-(anthracen-9-yl)phenolato]$\left.\kappa^{2} O: O ; \kappa^{2} O: O ; \kappa O: 1,2-\eta, \kappa O\right]-\{[2-($ anthracen-9-yl)phenolato- $\kappa O] y t t e r b i u m(I I)\}$ toluene trisolvate

Crystal data

$\left[\mathrm{Yb}_{2}\left(\mathrm{C}_{20} \mathrm{H}_{13} \mathrm{O}\right)_{5}\left(\mathrm{C}_{4} \mathrm{H}_{8} \mathrm{O}\right)_{2}\right] \cdot 3 \mathrm{C}_{7} \mathrm{H}_{7}$

$M_{r}=2113.20$

Monoclinic, $P 2_{1} / c$

$a=14.4612(7) \AA$

$b=20.8473(10) \AA$

$c=32.0864(15) \AA$

$\beta=95.508(1)^{\circ}$

$V=9628.6(8) \AA^{3}$

$Z=4$

\section{Data collection}

Bruker SMART CCD area detector diffractometer

Parallel, graphite monochromator Detector resolution: 8.3 pixels $\mathrm{mm}^{-1}$

phi and $\omega$ scans

Absorption correction: multi-scan

(SADABS; Bruker, 2002)

$T_{\min }=0.729, T_{\max }=0.826$

\section{Refinement}

Refinement on $F^{2}$

Least-squares matrix: full

$R\left[F^{2}>2 \sigma\left(F^{2}\right)\right]=0.048$

$w R\left(F^{2}\right)=0.115$

$S=1.03$

22107 reflections

1480 parameters

1509 restraints
$F(000)=4300$

$D_{\mathrm{x}}=1.458 \mathrm{Mg} \mathrm{m}^{-3}$

Mo $K \alpha$ radiation, $\lambda=0.71073 \AA$

Cell parameters from 9535 reflections

$\theta=2.3-29.7^{\circ}$

$\mu=1.99 \mathrm{~mm}^{-1}$

$T=83 \mathrm{~K}$

Fragment, orange

$0.4 \times 0.37 \times 0.1 \mathrm{~mm}$

109364 measured reflections

22107 independent reflections

15925 reflections with $I>2 \sigma(I)$

$R_{\text {int }}=0.067$

$\theta_{\max }=27.5^{\circ}, \theta_{\min }=1.4^{\circ}$

$h=-18 \rightarrow 18$

$k=-27 \rightarrow 27$

$l=-41 \rightarrow 41$

Primary atom site location: structure-invariant direct methods

Hydrogen site location: inferred from neighbouring sites

$\mathrm{H}$-atom parameters constrained

$w=1 /\left[\sigma^{2}\left(F_{\mathrm{o}}^{2}\right)+(0.046 P)^{2}+19.2482 P\right]$

where $P=\left(F_{\mathrm{o}}{ }^{2}+2 F_{\mathrm{c}}{ }^{2}\right) / 3$

$(\Delta / \sigma)_{\max }=0.002$ 
$\Delta \rho_{\max }=2.20 \mathrm{e} \AA^{-3}$

$\Delta \rho_{\min }=-0.93$ e $\AA^{-3}$

Special details

Experimental. The data collection nominally covered a full sphere of reciprocal space by a combination of 5 sets of $\omega$ scans each set at different $\varphi$ and/or $2 \theta$ angles and each scan (5 s exposure) covering $-0.3^{\circ}$ degrees in $\omega$. The crystal to detector distance was $5.035 \mathrm{~cm}$.

Geometry. All esds (except the esd in the dihedral angle between two 1.s. planes) are estimated using the full covariance matrix. The cell esds are taken into account individually in the estimation of esds in distances, angles and torsion angles; correlations between esds in cell parameters are only used when they are defined by crystal symmetry. An approximate (isotropic) treatment of cell esds is used for estimating esds involving l.s. planes.

Refinement. One bridging ligand O7-C1E-C20E/O7-C1F-C20F was disordered and modelled in two positions with occupancies 53:47\% using rigid groups and restraints (SADI, SIMU). A metal coordinated THF is also disordered and modelled in two positions with occupancy 54:46\% using restraints (SADI, SIMU). Three toluene solvent molecules are also present and they are all disordered over two positions and modelled with rigid groups and restraints (SIMU). C29 and $\mathrm{C} 43$ toluene molecules were modelled at 50\% occupancy each and the $\mathrm{C} 36$ toluene was modelled at 68:32\%.

Fractional atomic coordinates and isotropic or equivalent isotropic displacement parameters $\left(\AA^{2}\right)$

\begin{tabular}{|c|c|c|c|c|c|}
\hline & $x$ & $y$ & $z$ & $U_{\text {iso }} * / U_{\text {eq }}$ & Occ. $(<1)$ \\
\hline $\mathrm{Yb} 1$ & $0.18124(2)$ & $0.71235(2)$ & $0.43346(2)$ & $0.02267(6)$ & \\
\hline Yb2 & $0.29037(2)$ & $0.79160(2)$ & $0.36561(2)$ & $0.03018(6)$ & \\
\hline $\mathrm{O} 1$ & 0.1748 (2) & $0.70802(14)$ & $0.36503(9)$ & $0.0283(7)$ & \\
\hline $\mathrm{O} 2$ & $0.3311(2)$ & $0.73221(14)$ & $0.42697(9)$ & $0.0258(7)$ & \\
\hline $\mathrm{O} 3$ & $0.2033(2)$ & $0.61927(14)$ & $0.45529(10)$ & $0.0299(7)$ & \\
\hline $\mathrm{O} 4$ & $0.3243(2)$ & $0.87059(15)$ & $0.32598(10)$ & $0.0342(8)$ & \\
\hline $\mathrm{O} 5$ & $0.0260(2)$ & $0.68505(14)$ & $0.42582(11)$ & $0.0310(7)$ & \\
\hline $\mathrm{O} 7$ & 0.1808 (2) & $0.81575(14)$ & $0.41572(11)$ & $0.0316(7)$ & \\
\hline C1A & $0.2202(3)$ & $0.5562(2)$ & $0.45664(14)$ & $0.0230(9)$ & \\
\hline $\mathrm{C} 1 \mathrm{~B}$ & $0.4010(3)$ & $0.6888(2)$ & $0.43332(13)$ & $0.0243(9)$ & \\
\hline $\mathrm{C} 1 \mathrm{C}$ & 0.3547 (3) & $0.9243(2)$ & $0.31022(15)$ & $0.0312(11)$ & \\
\hline C1D & $0.1103(3)$ & $0.7079(2)$ & $0.33159(14)$ & $0.0293(10)$ & \\
\hline $\mathrm{C} 2 \mathrm{~A}$ & $0.2194(3)$ & $0.5200(2)$ & $0.42007(14)$ & $0.0281(10)$ & \\
\hline $\mathrm{H} 2 \mathrm{~A}$ & 0.205598 & 0.540354 & 0.393708 & $0.034 *$ & \\
\hline $\mathrm{C} 2 \mathrm{~B}$ & $0.3910(3)$ & $0.6280(2)$ & $0.41576(13)$ & $0.0262(10)$ & \\
\hline $\mathrm{H} 2 \mathrm{~B}$ & 0.336306 & 0.617916 & 0.398207 & $0.031 *$ & \\
\hline $\mathrm{C} 2 \mathrm{C}$ & $0.3508(4)$ & $0.9822(2)$ & 0.33235 (16) & $0.0382(12)$ & \\
\hline $\mathrm{H} 2 \mathrm{C}$ & 0.325719 & 0.982494 & 0.358645 & $0.046^{*}$ & \\
\hline C2D & 0.0412 (3) & $0.7548(2)$ & $0.32733(16)$ & $0.0337(11)$ & \\
\hline H2D & 0.037827 & 0.785929 & 0.348779 & $0.040^{*}$ & \\
\hline C3A & $0.2383(3)$ & $0.4554(2)$ & $0.42154(15)$ & $0.0292(10)$ & \\
\hline H3A & 0.240208 & 0.432251 & 0.396134 & $0.035^{*}$ & \\
\hline $\mathrm{C} 3 \mathrm{~B}$ & 0.4589 (4) & $0.5823(2)$ & $0.42333(15)$ & $0.0344(11)$ & \\
\hline H3B & 0.450623 & 0.540827 & 0.411314 & $0.041 *$ & \\
\hline $\mathrm{C} 3 \mathrm{C}$ & $0.3830(4)$ & $1.0389(3)$ & 0.31651 (19) & $0.0454(14)$ & \\
\hline $\mathrm{H} 3 \mathrm{C}$ & 0.379386 & 1.077455 & 0.332031 & $0.055^{*}$ & \\
\hline $\mathrm{C} 3 \mathrm{D}$ & $-0.0224(3)$ & $0.7567(3)$ & $0.29232(17)$ & $0.0404(13)$ & \\
\hline $\mathrm{H} 3 \mathrm{D}$ & -0.068675 & 0.789189 & 0.289812 & $0.048 *$ & \\
\hline $\mathrm{C} 4 \mathrm{~A}$ & $0.2543(3)$ & $0.4237(2)$ & $0.45887(16)$ & $0.0297(10)$ & \\
\hline H4A & 0.265221 & 0.378799 & 0.459615 & $0.036^{*}$ & \\
\hline
\end{tabular}




\begin{tabular}{|c|c|c|c|c|}
\hline C4B & $0.5393(4)$ & $0.5966(2)$ & $0.44839(17)$ & $0.0398(13)$ \\
\hline $\mathrm{H} 4 \mathrm{~B}$ & 0.585761 & 0.564845 & 0.454417 & $0.048 *$ \\
\hline $\mathrm{C} 4 \mathrm{C}$ & $0.4201(4)$ & $1.0401(3)$ & $0.27849(18)$ & $0.0483(15)$ \\
\hline $\mathrm{H} 4 \mathrm{C}$ & 0.441889 & 1.079102 & 0.267623 & $0.058^{*}$ \\
\hline C4D & $-0.0191(4)$ & 0.7118 & $0.26110(17)$ & $0.0447(14)$ \\
\hline H4D & -0.063324 & 0.712957 & 0.237212 & $0.054^{*}$ \\
\hline C5A & $0.2542(3)$ & $0.4586(2)$ & $0.49543(15)$ & $0.0265(10)$ \\
\hline $\mathrm{H} 5 \mathrm{~A}$ & 0.264527 & 0.436775 & 0.521460 & $0.032 *$ \\
\hline C5B & $0.5512(4)$ & $0.6578(2)$ & $0.46455(16)$ & $0.0365(12)$ \\
\hline H5B & 0.606686 & 0.667661 & 0.481640 & $0.044 *$ \\
\hline $\mathrm{C} 5 \mathrm{C}$ & $0.4250(4)$ & $0.9832(3)$ & $0.25653(17)$ & $0.0381(12)$ \\
\hline $\mathrm{H} 5 \mathrm{C}$ & 0.451028 & 0.983682 & 0.230439 & $0.046^{*}$ \\
\hline C5D & 0.0488 & 0.6650 & $0.26468(15)$ & $0.0390(13)$ \\
\hline H5D & 0.051138 & 0.634067 & 0.243042 & $0.047^{*}$ \\
\hline C6A & $0.2396(3)$ & $0.5247(2)$ & $0.49552(13)$ & $0.0224(9)$ \\
\hline C6B & $0.4846(3)$ & $0.7051(2)$ & $0.45660(14)$ & $0.0287(10)$ \\
\hline $\mathrm{C} 6 \mathrm{C}$ & 0.3934 & $0.9256(2)$ & $0.27131(15)$ & $0.0296(10)$ \\
\hline C6D & 0.1142 & $0.6624(2)$ & $0.29973(15)$ & $0.0321(11)$ \\
\hline C7A & 0.2433 & $0.5583(2)$ & $0.53633(14)$ & $0.0262(10)$ \\
\hline C7B & 0.5042 & $0.7732(2)$ & $0.46942(15)$ & $0.0278(10)$ \\
\hline $\mathrm{C} 7 \mathrm{C}$ & $0.4006(3)$ & $0.8661(2)$ & $0.24624(14)$ & $0.0296(10)$ \\
\hline C7D & $0.1882(4)$ & $0.6123(2)$ & $0.30329(14)$ & $0.0356(12)$ \\
\hline C8A & $0.1610(3)$ & $0.5697(2)$ & $0.55534(14)$ & $0.0254(10)$ \\
\hline $\mathrm{C} 8 \mathrm{~B}$ & $0.5094(3)$ & $0.7924(2)$ & $0.51160(15)$ & $0.0305(10)$ \\
\hline $\mathrm{C} 8 \mathrm{C}$ & 0.4885 & $0.8407(3)$ & $0.23907(15)$ & $0.0357(12)$ \\
\hline C8D & $0.1647(4)$ & 0.5464 & $0.30659(15)$ & $0.0424(14)$ \\
\hline C9A & 0.0715 & $0.5564(2)$ & $0.53416(15)$ & $0.0282(10)$ \\
\hline H9A & 0.067287 & 0.539731 & 0.506480 & $0.034 *$ \\
\hline C9B & $0.4927(4)$ & $0.7499(2)$ & $0.54503(16)$ & $0.0360(12)$ \\
\hline H9B & 0.479051 & 0.706123 & 0.538845 & $0.043 *$ \\
\hline $\mathrm{C} 9 \mathrm{C}$ & 0.5740 & $0.8687(3)$ & $0.25642(18)$ & $0.0495(15)$ \\
\hline $\mathrm{H} 9 \mathrm{C}$ & 0.573142 & 0.906888 & 0.272577 & $0.059 *$ \\
\hline C9D & $0.0715(5)$ & 0.5249 & $0.30686(17)$ & $0.0554(17)$ \\
\hline H9D & 0.022788 & 0.555632 & 0.305154 & $0.067 *$ \\
\hline $\mathrm{C} 10 \mathrm{~A}$ & $-0.0073(3)$ & $0.5668(2)$ & $0.55266(17)$ & $0.0370(12)$ \\
\hline $\mathrm{H} 10 \mathrm{~A}$ & -0.065892 & 0.556658 & 0.538218 & $0.044^{*}$ \\
\hline C10B & $0.4959(4)$ & 0.7701 & $0.58521(17)$ & $0.0411(13)$ \\
\hline H10B & 0.483752 & 0.740700 & 0.606650 & $0.049 *$ \\
\hline $\mathrm{C} 10 \mathrm{C}$ & $0.6576(4)$ & $0.8405(4)$ & $0.2497(2)$ & $0.070(2)$ \\
\hline $\mathrm{H} 10 \mathrm{C}$ & 0.713898 & 0.859279 & 0.261676 & $0.084 *$ \\
\hline C10D & $0.0501(6)$ & 0.4618 & $0.3095(2)$ & $0.074(2)$ \\
\hline H10D & -0.012709 & 0.448702 & 0.309959 & $0.089^{*}$ \\
\hline C11A & $-0.0025(4)$ & 0.5928 & $0.59322(18)$ & $0.0494(15)$ \\
\hline H11A & -0.058133 & 0.601387 & 0.605755 & $0.059 *$ \\
\hline C11B & $0.5171(4)$ & 0.8350 & $0.59548(18)$ & $0.0467(14)$ \\
\hline H11B & 0.519444 & 0.848887 & 0.623759 & $0.056^{*}$ \\
\hline $\mathrm{C} 11 \mathrm{C}$ & $0.6610(5)$ & $0.7844(4)$ & 0.2256 & $0.073(2)$ \\
\hline $\mathrm{H} 11 \mathrm{C}$ & 0.719516 & 0.766008 & 0.221414 & $0.088^{*}$ \\
\hline
\end{tabular}




\begin{tabular}{|c|c|c|c|c|}
\hline C11D & $0.1212(7)$ & $0.4163(3)$ & $0.3115(2)$ & 0.079 (3) \\
\hline H11D & 0.105318 & 0.372135 & 0.312091 & $0.095^{*}$ \\
\hline $\mathrm{C} 12 \mathrm{~A}$ & $0.0811(4)$ & $0.6056(3)$ & $0.61464(17)$ & $0.0460(14)$ \\
\hline H12A & 0.082792 & 0.622412 & 0.642221 & $0.055^{*}$ \\
\hline C12B & $0.5342(4)$ & $0.8771(2)$ & $0.56509(17)$ & $0.0408(13)$ \\
\hline H12B & 0.548462 & 0.920313 & 0.572544 & $0.049^{*}$ \\
\hline $\mathrm{C} 12 \mathrm{C}$ & $0.5837(5)$ & 0.7568 & $0.2085(2)$ & $0.0595(18)$ \\
\hline $\mathrm{H} 12 \mathrm{C}$ & 0.587669 & 0.719133 & 0.192080 & $0.071^{*}$ \\
\hline C12D & $0.2109(7)$ & 0.4327 & $0.31252(18)$ & $0.068(2)$ \\
\hline H12D & 0.257641 & 0.400583 & 0.315014 & $0.082 *$ \\
\hline C13A & $0.1657(3)$ & $0.5946(2)$ & $0.59707(15)$ & $0.0307(11)$ \\
\hline C13B & $0.5313(3)$ & 0.8585 & $0.52198(16)$ & $0.0324(11)$ \\
\hline $\mathrm{C} 13 \mathrm{C}$ & $0.4941(4)$ & 0.7833 & $0.21442(17)$ & $0.0423(13)$ \\
\hline C13D & $0.2362(5)$ & 0.4995 & $0.30984(15)$ & $0.0501(16)$ \\
\hline C14A & $0.2524(3)$ & $0.6079(2)$ & $0.61869(15)$ & $0.0325(11)$ \\
\hline H14A & 0.255160 & 0.624343 & 0.646389 & $0.039 *$ \\
\hline C14B & 0.5465 & $0.9017(2)$ & $0.49078(17)$ & 0.0359 \\
\hline H14B & 0.560763 & 0.944955 & 0.498053 & $0.043 *$ \\
\hline $\mathrm{C} 14 \mathrm{C}$ & 0.4140 & 0.7535 & $0.19803(16)$ & $0.0416(13)$ \\
\hline $\mathrm{H} 14 \mathrm{C}$ & 0.418457 & 0.715930 & 0.181564 & $0.050 *$ \\
\hline C14D & $0.3287(5)$ & 0.5190 & $0.31169(16)$ & $0.0512(17)$ \\
\hline H14D & 0.376095 & 0.487354 & 0.314696 & $0.061^{*}$ \\
\hline C15A & $0.3344(3)$ & $0.5974(2)$ & $0.60049(14)$ & $0.0257(10)$ \\
\hline C15B & $0.5416(3)$ & $0.8834(2)$ & $0.44901(16)$ & $0.0322(11)$ \\
\hline $\mathrm{C} 15 \mathrm{C}$ & $0.3274(4)$ & $0.7765(2)$ & $0.20477(15)$ & $0.0365(12)$ \\
\hline C15D & 0.3543 & $0.5837(2)$ & $0.30929(15)$ & $0.0392(13)$ \\
\hline C16A & 0.4228 & $0.6083(2)$ & $0.62278(16)$ & $0.0334(11)$ \\
\hline H16A & 0.426471 & 0.622217 & 0.651074 & $0.040^{*}$ \\
\hline $\mathrm{C} 16 \mathrm{~B}$ & $0.5574(3)$ & $0.9278(2)$ & $0.41616(18)$ & $0.0393(13)$ \\
\hline H16B & 0.571359 & 0.971221 & 0.423074 & $0.047^{*}$ \\
\hline $\mathrm{C} 16 \mathrm{C}$ & $0.2436(4)$ & 0.7443 & $0.18899(16)$ & $0.0401(13)$ \\
\hline $\mathrm{H} 16 \mathrm{C}$ & 0.247275 & 0.707169 & 0.172046 & $0.048^{*}$ \\
\hline C16D & 0.4480 & 0.6040 & $0.31152(16)$ & $0.0469(15)$ \\
\hline H16D & 0.495979 & 0.572902 & 0.315808 & $0.056^{*}$ \\
\hline C17A & 0.5018 & $0.5989(2)$ & $0.60417(16)$ & $0.0348(12)$ \\
\hline H17A & 0.560283 & 0.605757 & 0.619681 & $0.042 *$ \\
\hline C17B & $0.5527(4)$ & $0.9092(3)$ & $0.37576(18)$ & $0.0459(14)$ \\
\hline H17B & 0.561908 & 0.939713 & 0.354555 & $0.055^{*}$ \\
\hline $\mathrm{C} 17 \mathrm{C}$ & $0.1596(4)$ & 0.7658 & $0.19780(16)$ & $0.0405(13)$ \\
\hline $\mathrm{H} 17 \mathrm{C}$ & 0.105025 & 0.743373 & 0.187391 & $0.049 *$ \\
\hline C17D & $0.4714(4)$ & 0.6670 & $0.30769(16)$ & $0.0410(13)$ \\
\hline H17D & 0.534743 & 0.679667 & 0.310128 & $0.049 *$ \\
\hline C18A & $0.4984(3)$ & $0.5790(2)$ & $0.56188(17)$ & $0.0338(11)$ \\
\hline H18A & 0.554386 & 0.574206 & 0.548931 & $0.041 *$ \\
\hline C18B & $0.5342(4)$ & $0.8442(3)$ & $0.36484(17)$ & $0.0393(12)$ \\
\hline H18B & 0.532422 & 0.831041 & 0.336419 & $0.047^{*}$ \\
\hline $\mathrm{C} 18 \mathrm{C}$ & $0.1522(4)$ & $0.8218(2)$ & $0.22252(15)$ & $0.0351(11)$ \\
\hline $\mathrm{H} 18 \mathrm{C}$ & 0.092713 & 0.836194 & 0.228779 & $0.042 *$ \\
\hline
\end{tabular}




\begin{tabular}{|c|c|c|c|c|c|}
\hline C18D & $0.3992(3)$ & $0.7138(2)$ & $0.29995(16)$ & $0.0363(12)$ & \\
\hline H18D & 0.414763 & 0.757451 & 0.295968 & $0.044 *$ & \\
\hline C19A & 0.4149 (3) & $0.5667(2)$ & $0.53958(15)$ & $0.0285(10)$ & \\
\hline H19A & 0.413542 & 0.553101 & 0.511270 & $0.034 *$ & \\
\hline C19B & $0.5190(3)$ & $0.8005(2)$ & 0.39515 (16) & $0.0343(11)$ & \\
\hline H19B & 0.506915 & 0.757103 & 0.387344 & $0.041^{*}$ & \\
\hline $\mathrm{C} 19 \mathrm{C}$ & $0.2288(3)$ & $0.8547(2)$ & $0.23718(14)$ & $0.0299(10)$ & \\
\hline H19C & 0.222355 & 0.892880 & 0.252793 & $0.036^{*}$ & \\
\hline C19D & $0.3086(3)$ & $0.6960(2)$ & $0.29828(14)$ & $0.0310(11)$ & \\
\hline H19D & 0.261545 & 0.727653 & 0.293317 & $0.037^{*}$ & \\
\hline $\mathrm{C} 20 \mathrm{~A}$ & $0.3301(3)$ & $0.5740(2)$ & $0.55821(14)$ & $0.0245(9)$ & \\
\hline $\mathrm{C} 20 \mathrm{~B}$ & $0.5207(3)$ & $0.8180(2)$ & $0.43816(16)$ & $0.0295(10)$ & \\
\hline $\mathrm{C} 20 \mathrm{C}$ & 0.3199 (3) & $0.8331(2)$ & $0.22963(14)$ & $0.0278(10)$ & \\
\hline C20D & $0.2824(4)$ & $0.6306(2)$ & $0.30381(13)$ & $0.0314(11)$ & \\
\hline $\mathrm{C} 25$ & $-0.0049(4)$ & $0.6217(3)$ & 0.41039 (18) & $0.0445(14)$ & \\
\hline $\mathrm{H} 25 \mathrm{~A}$ & 0.040546 & 0.588411 & 0.420601 & $0.053^{*}$ & \\
\hline $\mathrm{H} 25 \mathrm{~B}$ & -0.011492 & 0.621062 & 0.379391 & $0.053^{*}$ & \\
\hline $\mathrm{C} 26$ & $-0.0948(4)$ & $0.6101(3)$ & $0.4266(2)$ & $0.0602(19)$ & \\
\hline H26A & -0.089571 & 0.574751 & 0.447253 & $0.072 *$ & \\
\hline $\mathrm{H} 26 \mathrm{~B}$ & -0.141968 & 0.598151 & 0.403439 & $0.072 *$ & \\
\hline $\mathrm{C} 27$ & $-0.1224(4)$ & $0.6716(3)$ & 0.44698 (19) & $0.0463(14)$ & \\
\hline $\mathrm{H} 27 \mathrm{~A}$ & -0.188132 & 0.682369 & 0.438375 & $0.056^{*}$ & \\
\hline H27B & -0.113473 & 0.668121 & 0.477879 & $0.056^{*}$ & \\
\hline C28 & $-0.0584(3)$ & $0.7209(2)$ & $0.43141(17)$ & 0.0355 (11) & \\
\hline $\mathrm{H} 28 \mathrm{~A}$ & -0.085145 & 0.739666 & 0.404579 & $0.043^{*}$ & \\
\hline H28B & -0.045856 & 0.755756 & 0.452139 & $0.043^{*}$ & \\
\hline $\mathrm{C} 43 \mathrm{~A}$ & $0.2303(7)$ & $0.2770(4)$ & $0.3876(4)$ & $0.078(3)$ & 0.5 \\
\hline $\mathrm{H} 43 \mathrm{~A}$ & 0.285362 & 0.280496 & 0.372109 & $0.117^{*}$ & 0.5 \\
\hline H43B & 0.249717 & 0.274269 & 0.417637 & $0.117^{*}$ & 0.5 \\
\hline $\mathrm{H} 43 \mathrm{C}$ & 0.191037 & 0.314970 & 0.382003 & $0.117^{*}$ & 0.5 \\
\hline $\mathrm{C} 44 \mathrm{~A}$ & $0.1795(5)$ & $0.2212(3)$ & $0.3745(3)$ & $0.0789(16)$ & 0.5 \\
\hline $\mathrm{C} 45 \mathrm{~A}$ & $0.0830(5)$ & $0.2218(4)$ & $0.3733(4)$ & $0.0774(18)$ & 0.5 \\
\hline $\mathrm{H} 45 \mathrm{~A}$ & 0.052084 & 0.258220 & 0.383074 & $0.093^{*}$ & 0.5 \\
\hline C46A & $0.0321(4)$ & $0.1689(5)$ & 0.3579 (4) & $0.0772(18)$ & 0.5 \\
\hline H46A & -0.033788 & 0.169338 & 0.357046 & $0.093^{*}$ & 0.5 \\
\hline C47A & $0.0773(6)$ & 0.1155 (4) & 0.3437 (3) & $0.0790(19)$ & 0.5 \\
\hline H47A & 0.042443 & 0.079426 & 0.333198 & $0.095^{*}$ & 0.5 \\
\hline C48A & $0.1736(6)$ & $0.1150(4)$ & $0.3450(4)$ & 0.0818 (19) & 0.5 \\
\hline H48A & 0.204598 & 0.078473 & 0.335265 & $0.098^{*}$ & 0.5 \\
\hline C49A & 0.2247 (4) & $0.1678(4)$ & $0.3604(3)$ & 0.0811 (19) & 0.5 \\
\hline H49A & 0.290571 & 0.167309 & 0.361316 & $0.097 *$ & 0.5 \\
\hline O6A & $0.1671(12)$ & $0.7551(5)$ & 0.4989 (2) & $0.0326(14)$ & $0.542(9)$ \\
\hline $\mathrm{C} 1 \mathrm{E}$ & $0.1884(4)$ & $0.8757(2)$ & $0.4304(2)$ & $0.0298(13)$ & $0.533(3)$ \\
\hline $\mathrm{C} 2 \mathrm{E}$ & 0.2801 (3) & 0.8939 (2) & $0.4410(2)$ & $0.0311(13)$ & $0.533(3)$ \\
\hline $\mathrm{H} 2 \mathrm{E}$ & 0.329155 & 0.864980 & 0.436773 & $0.037^{*}$ & $0.533(3)$ \\
\hline $\mathrm{C} 3 \mathrm{E}$ & 0.3001 (3) & $0.9543(2)$ & $0.4578(2)$ & $0.0342(13)$ & $0.533(3)$ \\
\hline H3E & 0.362761 & 0.966742 & 0.465054 & $0.041^{*}$ & 0.533 \\
\hline $\mathrm{C} 4 \mathrm{E}$ & $0.2283(4)$ & $0.9966(2)$ & $0.4640(2)$ & $0.0366(13)$ & 0.533 \\
\hline
\end{tabular}




\begin{tabular}{|c|c|c|c|c|c|}
\hline $\mathrm{H} 4 \mathrm{E}$ & 0.241967 & 1.037899 & 0.475464 & $0.044 *$ & $0.533(3)$ \\
\hline C5E & $0.1366(3)$ & $0.9784(3)$ & $0.4534(2)$ & $0.0352(13)$ & $0.533(3)$ \\
\hline H5E & 0.087566 & 1.007294 & 0.457594 & $0.042 *$ & $0.533(3)$ \\
\hline C6E & 0.1166 & $0.9180(3)$ & $0.4366(2)$ & $0.0305(11)$ & $0.533(3)$ \\
\hline C7E & $0.0168(2)$ & $0.8982(2)$ & $0.42281(11)$ & $0.0331(11)$ & $0.533(3)$ \\
\hline $\mathrm{C} 16 \mathrm{E}$ & $-0.1504(3)$ & 0.9075 & $0.32721(11)$ & $0.0493(14)$ & $0.533(3)$ \\
\hline $\mathrm{H} 16 \mathrm{E}$ & -0.214191 & 0.899831 & 0.318711 & $0.059 *$ & $0.533(3)$ \\
\hline C8E & $-0.0443(2)$ & 0.88209 (19) & $0.45364(10)$ & $0.0342(11)$ & $0.533(3)$ \\
\hline C9E & -0.0098 & $0.8764(3)$ & $0.49673(10)$ & $0.0377(13)$ & $0.533(3)$ \\
\hline H9E & 0.053294 & 0.886347 & 0.505337 & $0.045^{*}$ & $0.533(3)$ \\
\hline $\mathrm{C} 10 \mathrm{E}$ & -0.0681 & $0.8563(3)$ & $0.52590(10)$ & $0.0424(14)$ & $0.533(3)$ \\
\hline $\mathrm{H} 10 \mathrm{E}$ & -0.044746 & 0.852080 & 0.554468 & $0.051 *$ & $0.533(3)$ \\
\hline C11E & -0.1621 & $0.8421(3)$ & $0.51339(12)$ & $0.0440(15)$ & $0.533(3)$ \\
\hline H11E & -0.200939 & 0.827100 & 0.533557 & $0.053 *$ & $0.533(3)$ \\
\hline $\mathrm{C} 12 \mathrm{E}$ & $-0.1984(3)$ & $0.8497(3)$ & $0.47227(13)$ & $0.0414(13)$ & $0.533(3)$ \\
\hline $\mathrm{H} 12 \mathrm{E}$ & -0.262315 & 0.841122 & 0.464524 & $0.050 *$ & $0.533(3)$ \\
\hline C13E & $-0.1401(2)$ & $0.87029(18)$ & $0.44143(11)$ & $0.0384(11)$ & $0.533(3)$ \\
\hline C14E & $-0.1751(2)$ & $0.8800(2)$ & $0.39995(11)$ & $0.0414(13)$ & $0.533(3)$ \\
\hline H14E & -0.239550 & 0.874325 & 0.392097 & $0.050^{*}$ & $0.533(3)$ \\
\hline C15E & $-0.1172(2)$ & 0.89793 (19) & $0.36992(10)$ & $0.0422(12)$ & $0.533(3)$ \\
\hline C17E & -0.0947 (4) & $0.9269(3)$ & $0.29839(10)$ & $0.0534(14)$ & $0.533(3)$ \\
\hline H17E & -0.120476 & 0.935575 & 0.270603 & $0.064 *$ & $0.533(3)$ \\
\hline C18E & $0.0017(4)$ & $0.9347(3)$ & $0.30903(11)$ & $0.0522(14)$ & $0.533(3)$ \\
\hline $\mathrm{H} 18 \mathrm{E}$ & 0.040879 & 0.945904 & 0.288056 & $0.063^{*}$ & $0.533(3)$ \\
\hline C19E & 0.0380 & $0.9261(3)$ & $0.34878(12)$ & $0.0460(12)$ & $0.533(3)$ \\
\hline H19E & 0.102745 & 0.932243 & 0.355857 & $0.055^{*}$ & $0.533(3)$ \\
\hline $\mathrm{C} 20 \mathrm{E}$ & $-0.0206(2)$ & $0.90768(18)$ & $0.38094(10)$ & $0.0386(12)$ & $0.533(3)$ \\
\hline $\mathrm{C} 21 \mathrm{~A}$ & 0.1062 (17) & $0.7393(8)$ & $0.5294(8)$ & $0.0336(16)$ & $0.542(9)$ \\
\hline $\mathrm{H} 21 \mathrm{~A}$ & 0.099630 & 0.692188 & 0.531742 & $0.040^{*}$ & $0.542(9)$ \\
\hline $\mathrm{H} 21 \mathrm{~B}$ & 0.043947 & 0.758209 & 0.522054 & $0.040^{*}$ & $0.542(9)$ \\
\hline $\mathrm{C} 22 \mathrm{~A}$ & $0.1519(10)$ & $0.7681(8)$ & $0.5713(6)$ & $0.0350(16)$ & $0.542(9)$ \\
\hline $\mathrm{H} 22 \mathrm{~A}$ & 0.118518 & 0.807237 & 0.578808 & $0.042 *$ & $0.542(9)$ \\
\hline $\mathrm{H} 22 \mathrm{~B}$ & 0.150809 & 0.736586 & 0.594297 & $0.042 *$ & $0.542(9)$ \\
\hline $\mathrm{C} 23 \mathrm{~A}$ & $0.2433(11)$ & $0.7827(5)$ & $0.5640(5)$ & $0.0378(15)$ & $0.542(9)$ \\
\hline $\mathrm{H} 23 \mathrm{~A}$ & 0.263164 & 0.823478 & 0.577952 & $0.045^{*}$ & $0.542(9)$ \\
\hline $\mathrm{H} 23 \mathrm{~B}$ & 0.285993 & 0.748202 & 0.574952 & $0.045^{*}$ & $0.542(9)$ \\
\hline $\mathrm{C} 24 \mathrm{~A}$ & $0.2441(7)$ & $0.7882(5)$ & 0.5211 & $0.0358(14)$ & $0.542(9)$ \\
\hline $\mathrm{H} 24 \mathrm{~A}$ & 0.302918 & 0.770339 & 0.512721 & $0.043 *$ & $0.542(9)$ \\
\hline $\mathrm{H} 24 \mathrm{~B}$ & 0.241458 & 0.834147 & 0.513299 & $0.043^{*}$ & $0.542(9)$ \\
\hline C29A & $0.8741(6)$ & $0.0546(5)$ & $0.4107(2)$ & $0.054(2)$ & 0.5 \\
\hline $\mathrm{H} 29 \mathrm{~A}$ & 0.919939 & 0.022367 & 0.404242 & $0.081^{*}$ & 0.5 \\
\hline H29B & 0.844884 & 0.041392 & 0.435652 & $0.081 *$ & 0.5 \\
\hline $\mathrm{H} 29 \mathrm{C}$ & 0.905095 & 0.096032 & 0.415949 & $0.081 *$ & 0.5 \\
\hline $\mathrm{C} 30 \mathrm{~A}$ & $0.8051(4)$ & $0.0605(3)$ & $0.37630(16)$ & $0.0535(13)$ & 0.5 \\
\hline C31A & $0.8314(4)$ & $0.0589(4)$ & 0.33575 (19) & $0.0544(15)$ & 0.5 \\
\hline $\mathrm{H} 31 \mathrm{~A}$ & 0.895366 & 0.056982 & 0.331266 & $0.065^{*}$ & 0.5 \\
\hline $\mathrm{C} 32 \mathrm{~A}$ & $0.7641(5)$ & $0.0601(4)$ & $0.30176(15)$ & $0.0512(16)$ & 0.5 \\
\hline $\mathrm{H} 32 \mathrm{~A}$ & 0.781986 & 0.058994 & 0.274045 & $0.061 *$ & 0.5 \\
\hline
\end{tabular}




\begin{tabular}{|c|c|c|c|c|c|}
\hline C33A & $0.6705(5)$ & $0.0630(4)$ & $0.30839(19)$ & $0.0501(17)$ & 0.5 \\
\hline H33A & 0.624526 & 0.063737 & 0.285185 & $0.060^{*}$ & 0.5 \\
\hline C34A & $0.6443(4)$ & $0.0647(4)$ & 0.3489 (2) & $0.0507(15)$ & 0.5 \\
\hline H34A & 0.580405 & 0.066672 & 0.353414 & $0.061^{*}$ & 0.5 \\
\hline $\mathrm{C} 35 \mathrm{~A}$ & $0.7117(5)$ & $0.0635(4)$ & $0.38287(17)$ & $0.0517(15)$ & 0.5 \\
\hline $\mathrm{H} 35 \mathrm{~A}$ & 0.693817 & 0.064664 & 0.410585 & $0.062 *$ & 0.5 \\
\hline O6B & $0.1726(15)$ & $0.7423(7)$ & $0.5023(2)$ & $0.0334(15)$ & $0.458(9)$ \\
\hline $\mathrm{C} 5 \mathrm{~F}$ & $0.1475(4)$ & $0.9662(3)$ & $0.4715(2)$ & $0.0332(14)$ & $0.467(3)$ \\
\hline $\mathrm{H} 5 \mathrm{~F}$ & 0.098516 & 0.995934 & 0.473759 & $0.040^{*}$ & $0.467(3)$ \\
\hline $\mathrm{C} 6 \mathrm{~F}$ & 0.1324 (4) & $0.9115(3)$ & $0.4470(2)$ & $0.0316(12)$ & 0.467 (3) \\
\hline $\mathrm{C} 1 \mathrm{~F}$ & $0.2041(5)$ & $0.8679(3)$ & $0.4437(2)$ & $0.0313(13)$ & $0.467(3)$ \\
\hline $\mathrm{C} 2 \mathrm{~F}$ & 0.2909 (4) & $0.8790(3)$ & $0.4650(2)$ & $0.0311(13)$ & $0.467(3)$ \\
\hline $\mathrm{H} 2 \mathrm{~F}$ & 0.339930 & 0.849229 & 0.462773 & $0.037^{*}$ & $0.467(3)$ \\
\hline $\mathrm{C} 3 \mathrm{~F}$ & $0.3061(3)$ & $0.9336(3)$ & 0.4895 (2) & $0.0348(13)$ & $0.467(3)$ \\
\hline $\mathrm{H} 3 \mathrm{~F}$ & 0.365440 & 0.941209 & 0.504077 & $0.042 *$ & $0.467(3)$ \\
\hline $\mathrm{C} 4 \mathrm{~F}$ & $0.2344(4)$ & $0.9772(3)$ & $0.4928(2)$ & $0.0358(13)$ & $0.467(3)$ \\
\hline $\mathrm{H} 4 \mathrm{~F}$ & 0.244734 & 1.014562 & 0.509570 & $0.043^{*}$ & $0.467(3)$ \\
\hline $\mathrm{C} 7 \mathrm{~F}$ & $0.0397(2)$ & 0.9035 (3) & $0.42304(12)$ & $0.0324(11)$ & $0.467(3)$ \\
\hline $\mathrm{C} 8 \mathrm{~F}$ & $0.0316(3)$ & 0.9164 (2) & $0.37963(12)$ & $0.0395(13)$ & $0.467(3)$ \\
\hline $\mathrm{C} 9 \mathrm{~F}$ & $0.1117(3)$ & $0.9351(3)$ & $0.35855(15)$ & $0.0434(16)$ & $0.467(3)$ \\
\hline $\mathrm{H} 9 \mathrm{~F}$ & 0.170698 & 0.939064 & 0.374112 & $0.052^{*}$ & $0.467(3)$ \\
\hline $\mathrm{C} 10 \mathrm{~F}$ & 0.1032 (4) & 0.9469 (3) & $0.31718(15)$ & $0.0489(16)$ & $0.467(3)$ \\
\hline $\mathrm{H} 10 \mathrm{~F}$ & 0.156308 & 0.958297 & 0.303532 & $0.059^{*}$ & $0.467(3)$ \\
\hline $\mathrm{C} 11 \mathrm{~F}$ & $0.0154(4)$ & $0.9425(3)$ & $0.29398(12)$ & $0.0526(15)$ & $0.467(3)$ \\
\hline H11F & 0.009159 & 0.953431 & 0.265106 & $0.063^{*}$ & $0.467(3)$ \\
\hline $\mathrm{C} 12 \mathrm{~F}$ & $-0.0596(4)$ & $0.9229(3)$ & $0.31230(11)$ & $0.0511(14)$ & $0.467(3)$ \\
\hline $\mathrm{H} 12 \mathrm{~F}$ & -0.116776 & 0.917494 & 0.295496 & $0.061^{*}$ & $0.467(3)$ \\
\hline $\mathrm{C} 13 \mathrm{~F}$ & $-0.0561(3)$ & $0.9100(2)$ & $0.35594(11)$ & $0.0462(12)$ & $0.467(3)$ \\
\hline $\mathrm{C} 14 \mathrm{~F}$ & $-0.1340(2)$ & 0.8919 (3) & $0.37520(12)$ & $0.0431(13)$ & $0.467(3)$ \\
\hline $\mathrm{H} 14 \mathrm{~F}$ & -0.192309 & 0.888457 & 0.358977 & $0.052 *$ & $0.467(3)$ \\
\hline $\mathrm{C} 15 \mathrm{~F}$ & $-0.1281(2)$ & $0.8788(2)$ & $0.41792(13)$ & $0.0392(12)$ & $0.467(3)$ \\
\hline $\mathrm{C} 16 \mathrm{~F}$ & $-0.2071(3)$ & $0.8580(3)$ & $0.43773(16)$ & $0.0411(13)$ & $0.467(3)$ \\
\hline $\mathrm{H} 16 \mathrm{~F}$ & -0.264952 & 0.851699 & 0.421586 & $0.049^{*}$ & $0.467(3)$ \\
\hline $\mathrm{C} 17 \mathrm{~F}$ & $-0.1994(3)$ & $0.8471(3)$ & $0.48043(16)$ & $0.0423(13)$ & $0.467(3)$ \\
\hline $\mathrm{H} 17 \mathrm{~F}$ & -0.251809 & 0.831962 & 0.493255 & $0.051^{*}$ & $0.467(3)$ \\
\hline $\mathrm{C} 18 \mathrm{~F}$ & $-0.1149(4)$ & $0.8580(3)$ & $0.50526(13)$ & $0.0412(13)$ & $0.467(3)$ \\
\hline $\mathrm{H} 18 \mathrm{~F}$ & -0.111426 & 0.851555 & 0.534677 & $0.049^{*}$ & $0.467(3)$ \\
\hline $\mathrm{C} 19 \mathrm{~F}$ & $-0.0371(3)$ & $0.8781(3)$ & $0.48692(11)$ & $0.0376(12)$ & $0.467(3)$ \\
\hline $\mathrm{H} 19 \mathrm{~F}$ & 0.019443 & 0.885828 & 0.503775 & $0.045^{*}$ & $0.467(3)$ \\
\hline $\mathrm{C} 20 \mathrm{~F}$ & $-0.0418(3)$ & $0.8872(2)$ & $0.44263(11)$ & $0.0352(11)$ & 0.467 (3) \\
\hline $\mathrm{C} 21 \mathrm{~B}$ & $0.101(2)$ & $0.7283(10)$ & $0.5294(9)$ & $0.0332(16)$ & $0.458(9)$ \\
\hline $\mathrm{H} 21 \mathrm{C}$ & 0.093124 & 0.681387 & 0.532219 & $0.040^{*}$ & $0.458(9)$ \\
\hline H21D & 0.041345 & 0.747483 & 0.518307 & $0.040^{*}$ & $0.458(9)$ \\
\hline $\mathrm{C} 22 \mathrm{~B}$ & $0.1356(12)$ & $0.7576(10)$ & $0.5704(7)$ & $0.0347(16)$ & $0.458(9)$ \\
\hline $\mathrm{H} 22 \mathrm{C}$ & 0.122000 & 0.729794 & 0.594090 & $0.042^{*}$ & $0.458(9)$ \\
\hline $\mathrm{H} 22 \mathrm{D}$ & 0.107506 & 0.800380 & 0.573892 & $0.042 *$ & $0.458(9)$ \\
\hline C23B & $0.2472(13)$ & $0.7632(7)$ & $0.5672(5)$ & $0.0372(15)$ & $0.458(9)$ \\
\hline $\mathrm{H} 23 \mathrm{C}$ & 0.267779 & 0.808327 & 0.570983 & $0.045^{*}$ & $0.458(9)$ \\
\hline
\end{tabular}




\begin{tabular}{|c|c|c|c|c|c|}
\hline $\mathrm{H} 23 \mathrm{D}$ & 0.281624 & 0.736683 & 0.589046 & $0.045^{*}$ & $0.458(9)$ \\
\hline $\mathrm{C} 24 \mathrm{~B}$ & $0.2628(8)$ & $0.7424(6)$ & $0.5292(3)$ & $0.0356(14)$ & $0.458(9)$ \\
\hline $\mathrm{H} 24 \mathrm{C}$ & 0.289072 & 0.698481 & 0.531100 & $0.043^{*}$ & $0.458(9)$ \\
\hline $\mathrm{H} 24 \mathrm{D}$ & 0.307895 & 0.770831 & 0.516952 & $0.043^{*}$ & $0.458(9)$ \\
\hline C29B & $0.7034(7)$ & $0.0753(5)$ & $0.30219(18)$ & $0.055(2)$ & 0.5 \\
\hline H29D & 0.721217 & 0.117645 & 0.292322 & $0.083^{*}$ & 0.5 \\
\hline H29E & 0.635575 & 0.072810 & 0.301380 & $0.083^{*}$ & 0.5 \\
\hline $\mathrm{H} 29 \mathrm{~F}$ & 0.725805 & 0.042055 & 0.284074 & $0.083^{*}$ & 0.5 \\
\hline $\mathrm{C} 30 \mathrm{~B}$ & $0.7433(5)$ & 0.0659 & $0.34387(16)$ & $0.0528(13)$ & 0.5 \\
\hline $\mathrm{C} 31 \mathrm{~B}$ & $0.8396(4)$ & $0.0661(4)$ & $0.3523(2)$ & $0.0555(15)$ & 0.5 \\
\hline H31B & 0.877985 & 0.069540 & 0.329945 & $0.067 *$ & 0.5 \\
\hline $\mathrm{C} 32 \mathrm{~B}$ & $0.8794(4)$ & $0.0614(5)$ & $0.3933(2)$ & $0.0601(17)$ & 0.5 \\
\hline H32B & 0.945100 & 0.061571 & 0.399046 & $0.072 *$ & 0.5 \\
\hline C33B & $0.8233(6)$ & $0.0564(4)$ & $0.42596(18)$ & $0.0609(18)$ & 0.5 \\
\hline H33B & 0.850582 & 0.053133 & 0.454015 & $0.073 *$ & 0.5 \\
\hline C34B & $0.7271(6)$ & $0.0561(4)$ & $0.41757(18)$ & $0.0587(16)$ & 0.5 \\
\hline H34B & 0.688766 & 0.052728 & 0.439896 & $0.070^{*}$ & 0.5 \\
\hline C35B & $0.6872(4)$ & $0.0607(4)$ & $0.3765(2)$ & $0.0538(15)$ & 0.5 \\
\hline H35B & 0.621541 & 0.060394 & 0.370797 & $0.065^{*}$ & 0.5 \\
\hline C43B & $0.1304(7)$ & $0.0836(4)$ & $0.3527(4)$ & $0.081(3)$ & 0.5 \\
\hline H43D & 0.071015 & 0.068067 & 0.361083 & $0.121 *$ & 0.5 \\
\hline $\mathrm{H} 43 \mathrm{E}$ & 0.181073 & 0.057203 & 0.365898 & $0.121^{*}$ & 0.5 \\
\hline $\mathrm{H} 43 \mathrm{~F}$ & 0.129904 & 0.080796 & 0.322143 & $0.121 *$ & 0.5 \\
\hline C44B & $0.1438(5)$ & 0.1484 & $0.3654(3)$ & 0.0777 (16) & 0.5 \\
\hline $\mathrm{C} 45 \mathrm{~B}$ & $0.0688(4)$ & 0.1905 (4) & $0.3615(3)$ & 0.0757 (18) & 0.5 \\
\hline $\mathrm{H} 45 \mathrm{~B}$ & 0.010378 & 0.176639 & 0.348477 & $0.091 *$ & 0.5 \\
\hline $\mathrm{C} 46 \mathrm{~B}$ & $0.0794(6)$ & 0.2528 & $0.3765(4)$ & $0.0775(18)$ & 0.5 \\
\hline H46B & 0.028184 & 0.281606 & 0.373789 & $0.093 *$ & 0.5 \\
\hline C47B & $0.1648(7)$ & $0.2731(3)$ & $0.3955(3)$ & 0.0792 (19) & 0.5 \\
\hline H47B & 0.172037 & 0.315759 & 0.405742 & $0.095 *$ & 0.5 \\
\hline C48B & $0.2397(5)$ & $0.2311(4)$ & $0.3994(3)$ & 0.0825 (19) & 0.5 \\
\hline H48B & 0.298118 & 0.244925 & 0.412463 & $0.099 *$ & 0.5 \\
\hline C49B & $0.2292(4)$ & $0.1687(4)$ & 0.3843 & 0.0801 (19) & 0.5 \\
\hline H49B & 0.280413 & 0.140009 & 0.386974 & $0.096^{*}$ & 0.5 \\
\hline $\mathrm{C} 36 \mathrm{~B}$ & $0.7321(12)$ & $0.7340(7)$ & $0.3380(6)$ & $0.061(4)$ & $0.318(9)$ \\
\hline H36A & 0.690295 & 0.750905 & 0.314685 & $0.091 *$ & $0.318(9)$ \\
\hline H36B & 0.793497 & 0.753729 & 0.337608 & $0.091^{*}$ & $0.318(9)$ \\
\hline $\mathrm{H} 36 \mathrm{C}$ & 0.706938 & 0.743938 & 0.364511 & $0.091^{*}$ & $0.318(9)$ \\
\hline C37B & $0.7400(9)$ & $0.6665(7)$ & $0.3337(5)$ & 0.0595 (19) & $0.318(9)$ \\
\hline C38B & 0.6967 (11) & $0.6265(7)$ & $0.3606(5)$ & 0.0584 (19) & $0.318(9)$ \\
\hline H38B & 0.665933 & 0.644285 & 0.382790 & $0.070^{*}$ & $0.318(9)$ \\
\hline C39B & $0.6986(13)$ & $0.5604(7)$ & $0.3550(6)$ & $0.058(2)$ & $0.318(9)$ \\
\hline H39B & 0.669008 & 0.533062 & 0.373378 & $0.070^{*}$ & $0.318(9)$ \\
\hline $\mathrm{C} 40 \mathrm{~B}$ & $0.7437(13)$ & $0.5343(7)$ & $0.3226(6)$ & $0.060(2)$ & $0.318(9)$ \\
\hline H40B & 0.744901 & 0.489150 & 0.318789 & $0.072 *$ & $0.318(9)$ \\
\hline C41B & $0.7870(13)$ & $0.5743(8)$ & $0.2958(6)$ & $0.061(2)$ & $0.318(9)$ \\
\hline H41B & 0.817800 & 0.556526 & 0.273635 & $0.073 *$ & $0.318(9)$ \\
\hline C42B & $0.7852(11)$ & $0.6404(8)$ & $0.3014(5)$ & $0.061(2)$ & $0.318(9)$ \\
\hline
\end{tabular}




\begin{tabular}{llllll} 
H42B & 0.815030 & 0.667762 & 0.283170 & $0.073^{*}$ & $0.318(9)$ \\
C36A & $0.8116(6)$ & $0.5568(4)$ & $0.2732(2)$ & $0.071(3)$ & $0.682(9)$ \\
H36D & 0.764253 & 0.549913 & 0.249635 & $0.106^{*}$ & $0.682(9)$ \\
H36E & 0.839255 & 0.515504 & 0.282132 & $0.106^{*}$ & $0.682(9)$ \\
H36F & 0.860064 & 0.585252 & 0.264408 & $0.106^{*}$ & $0.682(9)$ \\
C37A & $0.7700(4)$ & $0.5852(3)$ & $0.3070(2)$ & $0.0593(18)$ & $0.682(9)$ \\
C38A & $0.7716(5)$ & $0.6516(3)$ & $0.3114(2)$ & $0.0612(18)$ & $0.682(9)$ \\
H38A & 0.804067 & 0.677262 & 0.293139 & $0.073^{*}$ & $0.682(9)$ \\
C39A & $0.7257(6)$ & $0.6804(3)$ & $0.3426(3)$ & $0.0604(18)$ & $0.682(9)$ \\
H39A & 0.726664 & 0.725716 & 0.345658 & $0.072^{*}$ & $0.682(9)$ \\
C40A & $0.6782(5)$ & $0.6428(4)$ & $0.3694(2)$ & $0.0575(18)$ & $0.682(9)$ \\
H40A & 0.646722 & 0.662541 & 0.390686 & $0.069^{*}$ & $0.682(9)$ \\
C41A & $0.6767(6)$ & $0.5765(4)$ & $0.3649(2)$ & $0.0573(19)$ & $0.682(9)$ \\
H41A & 0.644326 & 0.550861 & 0.383226 & $0.069^{*}$ & $0.682(9)$ \\
C42A & $0.7228(5)$ & $0.5477(3)$ & $0.3337(2)$ & $0.0580(18)$ & $0.682(9)$ \\
H42A & 0.721925 & 0.502409 & 0.330759 & $0.070^{*}$ & $0.682(9)$ \\
\hline
\end{tabular}

Atomic displacement parameters $\left(\AA^{2}\right)$

\begin{tabular}{|c|c|c|c|c|c|c|}
\hline & $U^{11}$ & $U^{22}$ & $U^{33}$ & $U^{12}$ & $U^{13}$ & $U^{23}$ \\
\hline Yb1 & $0.02982(11)$ & $0.01947(9)$ & $0.01901(10)$ & $-0.00670(8)$ & $0.00379(7)$ & $-0.00032(8)$ \\
\hline $\mathrm{Yb} 2$ & $0.03577(12)$ & $0.02727(11)$ & $0.02896(12)$ & $0.00081(9)$ & $0.01064(9)$ & $0.00974(9)$ \\
\hline $\mathrm{O} 1$ & $0.0348(18)$ & $0.0316(17)$ & $0.0184(16)$ & $-0.0016(14)$ & $0.0016(13)$ & $-0.0015(13)$ \\
\hline $\mathrm{O} 2$ & $0.0285(17)$ & $0.0243(15)$ & $0.0246(17)$ & $-0.0046(13)$ & $0.0026(13)$ & $0.0006(13)$ \\
\hline $\mathrm{O} 3$ & 0.0414 (19) & $0.0247(16)$ & $0.0229(17)$ & $-0.0058(14)$ & $-0.0004(14)$ & 0.0069 (13) \\
\hline $\mathrm{O} 4$ & $0.047(2)$ & $0.0305(17)$ & $0.0273(18)$ & $-0.0004(15)$ & $0.0120(15)$ & $0.0082(14)$ \\
\hline $\mathrm{O} 5$ & $0.0337(18)$ & $0.0226(15)$ & $0.037(2)$ & $-0.0062(14)$ & $0.0064(15)$ & $-0.0049(14)$ \\
\hline $\mathrm{O} 7$ & $0.0348(18)$ & $0.0205(15)$ & $0.041(2)$ & $-0.0051(13)$ & $0.0140(15)$ & $-0.0025(14)$ \\
\hline $\mathrm{C} 1 \mathrm{~A}$ & $0.021(2)$ & $0.022(2)$ & $0.026(2)$ & $-0.0062(17)$ & $0.0017(18)$ & $0.0034(18)$ \\
\hline C1B & $0.032(2)$ & $0.025(2)$ & $0.016(2)$ & $-0.0029(18)$ & $0.0038(18)$ & 0.0027 (17) \\
\hline $\mathrm{C} 1 \mathrm{C}$ & $0.036(3)$ & $0.028(2)$ & $0.029(3)$ & $-0.003(2)$ & $-0.002(2)$ & $0.007(2)$ \\
\hline C1D & $0.031(2)$ & $0.036(3)$ & $0.022(2)$ & $-0.010(2)$ & 0.0046 (19) & $0.006(2)$ \\
\hline $\mathrm{C} 2 \mathrm{~A}$ & $0.030(2)$ & $0.032(2)$ & $0.021(2)$ & $-0.009(2)$ & $-0.0021(19)$ & $-0.0002(19)$ \\
\hline $\mathrm{C} 2 \mathrm{~B}$ & $0.031(2)$ & $0.029(2)$ & $0.017(2)$ & $-0.0043(19)$ & $-0.0030(18)$ & $0.0011(18)$ \\
\hline $\mathrm{C} 2 \mathrm{C}$ & $0.045(3)$ & $0.042(3)$ & $0.027(3)$ & $0.000(2)$ & $0.001(2)$ & $0.002(2)$ \\
\hline $\mathrm{C} 2 \mathrm{D}$ & $0.030(3)$ & $0.040(3)$ & $0.032(3)$ & $-0.005(2)$ & $0.004(2)$ & $0.006(2)$ \\
\hline $\mathrm{C} 3 \mathrm{~A}$ & $0.020(2)$ & $0.034(2)$ & $0.034(3)$ & $-0.0101(19)$ & 0.0027 (19) & $-0.008(2)$ \\
\hline $\mathrm{C} 3 \mathrm{~B}$ & $0.042(3)$ & $0.029(2)$ & $0.031(3)$ & $0.000(2)$ & $-0.003(2)$ & $-0.007(2)$ \\
\hline $\mathrm{C} 3 \mathrm{C}$ & $0.053(4)$ & $0.034(3)$ & $0.048(4)$ & $-0.009(3)$ & $-0.005(3)$ & $-0.001(3)$ \\
\hline C3D & $0.027(3)$ & $0.054(3)$ & $0.041(3)$ & $-0.002(2)$ & $0.004(2)$ & $0.010(3)$ \\
\hline $\mathrm{C} 4 \mathrm{~A}$ & $0.019(2)$ & $0.025(2)$ & 0.045 (3) & 0.0007 (18) & $0.003(2)$ & $0.001(2)$ \\
\hline C4B & $0.036(3)$ & $0.032(3)$ & $0.049(3)$ & $0.005(2)$ & $-0.011(2)$ & $-0.005(2)$ \\
\hline $\mathrm{C} 4 \mathrm{C}$ & $0.059(4)$ & $0.039(3)$ & $0.046(4)$ & $-0.024(3)$ & $0.003(3)$ & $0.011(3)$ \\
\hline C4D & $0.029(3)$ & $0.073(4)$ & $0.031(3)$ & $-0.010(3)$ & $-0.002(2)$ & $0.010(3)$ \\
\hline C5A & $0.019(2)$ & $0.030(2)$ & $0.030(3)$ & $-0.0036(18)$ & $-0.0012(18)$ & $0.013(2)$ \\
\hline C5B & $0.034(3)$ & $0.031(3)$ & $0.041(3)$ & $-0.003(2)$ & $-0.011(2)$ & $-0.001(2)$ \\
\hline $\mathrm{C} 5 \mathrm{C}$ & $0.038(3)$ & $0.045(3)$ & $0.032(3)$ & $-0.013(2)$ & $0.004(2)$ & $0.009(2)$ \\
\hline C5D & $0.042(3)$ & $0.054(3)$ & $0.021(3)$ & $-0.022(3)$ & $0.006(2)$ & $-0.006(2)$ \\
\hline
\end{tabular}




\begin{tabular}{|c|c|c|c|c|c|c|}
\hline C6A & $0.017(2)$ & $0.033(2)$ & $0.016(2)$ & $-0.0041(17)$ & $-0.0027(16)$ & $0.0038(18)$ \\
\hline C6B & $0.035(3)$ & $0.027(2)$ & $0.023(2)$ & $-0.006(2)$ & -0.0019 (19) & $-0.0021(19)$ \\
\hline $\mathrm{C} 6 \mathrm{C}$ & 0.030 & $0.033(2)$ & $0.026(2)$ & -0.008 & $0.001(2)$ & $0.009(2)$ \\
\hline C6D & $0.039(3)$ & $0.035(3)$ & $0.023(2)$ & $-0.010(2)$ & $0.006(2)$ & $0.002(2)$ \\
\hline C7A & $0.026(2)$ & $0.028(2)$ & $0.024(2)$ & $-0.0073(18)$ & $-0.0021(19)$ & $0.0026(19)$ \\
\hline C7B & $0.026(2)$ & $0.026(2)$ & $0.030(3)$ & $-0.0026(18)$ & $-0.0028(19)$ & $0.0011(19)$ \\
\hline $\mathrm{C} 7 \mathrm{C}$ & 0.033 & $0.037(3)$ & $0.019(2)$ & $-0.004(2)$ & $0.0006(19)$ & $0.010(2)$ \\
\hline C7D & $0.059(3)$ & $0.031(3)$ & $0.017(2)$ & $-0.004(2)$ & $0.006(2)$ & -0.0015 (19) \\
\hline C8A & $0.030(2)$ & $0.025(2)$ & $0.021(2)$ & $-0.0072(18)$ & $-0.0007(18)$ & $0.0013(18)$ \\
\hline $\mathrm{C} 8 \mathrm{~B}$ & $0.028(2)$ & $0.030(2)$ & $0.032(3)$ & $-0.005(2)$ & $-0.004(2)$ & $-0.002(2)$ \\
\hline $\mathrm{C} 8 \mathrm{C}$ & $0.031(3)$ & $0.052(3)$ & $0.025(3)$ & $-0.004(2)$ & $0.004(2)$ & $0.018(2)$ \\
\hline $\mathrm{C} 8 \mathrm{D}$ & $0.076(4)$ & $0.033(3)$ & $0.018(2)$ & $-0.004(3)$ & $0.003(2)$ & $-0.001(2)$ \\
\hline C9A & $0.033(3)$ & $0.025(2)$ & $0.027(2)$ & $-0.0094(19)$ & $0.000(2)$ & $-0.0039(19)$ \\
\hline $\mathrm{C} 9 \mathrm{~B}$ & $0.041(3)$ & $0.030(3)$ & $0.035(3)$ & $-0.011(2)$ & $-0.005(2)$ & $-0.001(2)$ \\
\hline $\mathrm{C} 9 \mathrm{C}$ & 0.035 & $0.068(4)$ & $0.046(3)$ & $-0.004(3)$ & $0.005(3)$ & $0.024(3)$ \\
\hline C9D & $0.093(5)$ & $0.049(3)$ & $0.024(3)$ & $-0.025(3)$ & $0.000(3)$ & $-0.002(2)$ \\
\hline C10A & $0.024(2)$ & $0.044(3)$ & $0.043(3)$ & $-0.009(2)$ & $0.003(2)$ & $-0.016(2)$ \\
\hline $\mathrm{C} 10 \mathrm{~B}$ & $0.053(3)$ & 0.038 & $0.031(3)$ & $-0.017(2)$ & $-0.003(2)$ & $-0.002(2)$ \\
\hline $\mathrm{C} 10 \mathrm{C}$ & 0.033 & $0.102(6)$ & $0.076(5)$ & -0.005 & $0.010(3)$ & $0.028(5)$ \\
\hline C10D & $0.130(7)$ & $0.053(4)$ & $0.039(4)$ & $-0.040(5)$ & $-0.001(4)$ & $0.001(3)$ \\
\hline C11A & $0.032(3)$ & $0.071(4)$ & $0.047(4)$ & $-0.012(3)$ & $0.016(3)$ & $-0.025(3)$ \\
\hline C11B & $0.060(4)$ & $0.044(3)$ & $0.034(3)$ & $-0.015(3)$ & $-0.001(3)$ & $-0.011(3)$ \\
\hline $\mathrm{C} 11 \mathrm{C}$ & $0.047(4)$ & $0.085(5)$ & $0.093(6)$ & $0.019(4)$ & $0.028(4)$ & $0.026(5)$ \\
\hline C11D & $0.159(9)$ & $0.042(4)$ & $0.032(4)$ & $-0.034(5)$ & $-0.010(4)$ & $-0.008(3)$ \\
\hline $\mathrm{C} 12 \mathrm{~A}$ & $0.038(3)$ & $0.067(4)$ & $0.034(3)$ & $-0.017(3)$ & $0.010(2)$ & $-0.022(3)$ \\
\hline C12B & $0.048(3)$ & $0.030(3)$ & $0.043(3)$ & $-0.012(2)$ & $0.000(3)$ & $-0.011(2)$ \\
\hline $\mathrm{C} 12 \mathrm{C}$ & $0.055(4)$ & $0.062(4)$ & $0.066(5)$ & $0.015(3)$ & $0.029(3)$ & $0.015(3)$ \\
\hline C12D & $0.144(8)$ & $0.033(3)$ & $0.025(3)$ & $-0.001(4)$ & $-0.002(4)$ & $-0.002(2)$ \\
\hline C13A & $0.030(3)$ & $0.034(3)$ & $0.027(3)$ & $-0.011(2)$ & $0.002(2)$ & $-0.008(2)$ \\
\hline C13B & $0.031(3)$ & $0.026(2)$ & $0.038(3)$ & $-0.008(2)$ & $-0.004(2)$ & $-0.005(2)$ \\
\hline $\mathrm{C} 13 \mathrm{C}$ & $0.044(3)$ & $0.054(3)$ & $0.031(3)$ & $0.008(3)$ & $0.015(2)$ & $0.019(3)$ \\
\hline C13D & $0.100(5)$ & $0.035(3)$ & $0.014(2)$ & $0.001(3)$ & $-0.001(3)$ & $-0.006(2)$ \\
\hline C14A & $0.034(3)$ & $0.037(3)$ & $0.026(3)$ & $-0.008(2)$ & $-0.003(2)$ & $-0.006(2)$ \\
\hline C14B & $0.030(3)$ & $0.028(2)$ & $0.049(3)$ & $-0.008(2)$ & $-0.004(2)$ & $-0.003(2)$ \\
\hline $\mathrm{C} 14 \mathrm{C}$ & $0.056(4)$ & $0.045(3)$ & $0.026(3)$ & $0.006(3)$ & $0.016(2)$ & $0.006(2)$ \\
\hline C14D & $0.100(5)$ & $0.036(3)$ & $0.018(3)$ & $0.027(3)$ & $0.002(3)$ & $-0.005(2)$ \\
\hline C15A & 0.029 (2) & $0.022(2)$ & $0.025(2)$ & $-0.0066(18)$ & $-0.0052(19)$ & $0.0023(18)$ \\
\hline C15B & $0.027(2)$ & $0.030(2)$ & $0.039(3)$ & $-0.0036(19)$ & $-0.001(2)$ & $0.003(2)$ \\
\hline $\mathrm{C} 15 \mathrm{C}$ & $0.053(3)$ & $0.036(3)$ & $0.021(2)$ & $0.000(2)$ & $0.006(2)$ & $0.010(2)$ \\
\hline C15D & $0.061(4)$ & $0.039(3)$ & $0.016(2)$ & 0.018 & $0.000(2)$ & $-0.003(2)$ \\
\hline C16A & $0.036(3)$ & $0.029(2)$ & $0.032(3)$ & $-0.008(2)$ & $-0.011(2)$ & $-0.002(2)$ \\
\hline C16B & $0.036(3)$ & $0.030(3)$ & $0.051(3)$ & $-0.009(2)$ & $-0.001(2)$ & $0.007(2)$ \\
\hline $\mathrm{C} 16 \mathrm{C}$ & $0.062(4)$ & $0.036(3)$ & $0.022(3)$ & $-0.012(3)$ & $0.003(2)$ & $0.002(2)$ \\
\hline C16D & $0.060(4)$ & $0.060(4)$ & $0.021(3)$ & $0.033(3)$ & $0.002(2)$ & $-0.001(2)$ \\
\hline C17A & 0.028 & $0.030(2)$ & $0.042(3)$ & $-0.007(2)$ & $-0.016(2)$ & $0.002(2)$ \\
\hline C17B & $0.048(3)$ & $0.047(3)$ & $0.041(3)$ & $-0.014(3)$ & $-0.004(3)$ & $0.017(3)$ \\
\hline $\mathrm{C} 17 \mathrm{C}$ & $0.046(3)$ & $0.044(3)$ & $0.029(3)$ & -0.019 & $-0.009(2)$ & $0.010(2)$ \\
\hline C17D & $0.036(3)$ & $0.061(4)$ & $0.027(3)$ & $0.020(3)$ & $0.005(2)$ & $0.002(2)$ \\
\hline
\end{tabular}




\begin{tabular}{|c|c|c|c|c|c|c|}
\hline C18A & $0.027(2)$ & $0.028(2)$ & $0.045(3)$ & $-0.0039(19)$ & $-0.004(2)$ & $0.002(2)$ \\
\hline C18B & $0.041(3)$ & $0.044(3)$ & $0.032(3)$ & $-0.010(2)$ & $-0.002(2)$ & $0.006(2)$ \\
\hline $\mathrm{C} 18 \mathrm{C}$ & $0.034(3)$ & $0.042(3)$ & $0.028(3)$ & $-0.007(2)$ & $-0.002(2)$ & $0.012(2)$ \\
\hline C18D & $0.037(3)$ & $0.040(3)$ & $0.034(3)$ & $0.007(2)$ & $0.013(2)$ & $0.010(2)$ \\
\hline C19A & $0.026(2)$ & $0.028(2)$ & $0.030(3)$ & $-0.0038(19)$ & $-0.0020(19)$ & $0.000(2)$ \\
\hline C19B & $0.031(3)$ & $0.034(3)$ & $0.036(3)$ & $-0.006(2)$ & $-0.003(2)$ & $0.002(2)$ \\
\hline C19C & $0.035(3)$ & $0.032(2)$ & $0.022(2)$ & $-0.002(2)$ & $0.000(2)$ & 0.0094 (19) \\
\hline C19D & $0.037(3)$ & $0.037(3)$ & $0.020(2)$ & $0.009(2)$ & $0.005(2)$ & 0.0039 (19) \\
\hline $\mathrm{C} 20 \mathrm{~A}$ & $0.025(2)$ & $0.021(2)$ & $0.027(2)$ & $-0.0032(17)$ & $-0.0031(18)$ & $0.0075(18)$ \\
\hline $\mathrm{C} 20 \mathrm{~B}$ & $0.025(2)$ & $0.028(2)$ & $0.035(3)$ & $-0.0022(19)$ & $-0.001(2)$ & $0.000(2)$ \\
\hline $\mathrm{C} 20 \mathrm{C}$ & $0.034(3)$ & $0.032(2)$ & $0.017(2)$ & $-0.001(2)$ & $0.0015(19)$ & $0.0083(18)$ \\
\hline C20D & $0.051(3)$ & $0.030(2)$ & $0.013(2)$ & $0.008(2)$ & $0.002(2)$ & $0.0004(18)$ \\
\hline $\mathrm{C} 25$ & $0.052(3)$ & $0.038(3)$ & $0.044(3)$ & $-0.020(3)$ & 0.009 (3) & $-0.017(2)$ \\
\hline $\mathrm{C} 26$ & $0.032(3)$ & $0.029(3)$ & $0.119(6)$ & $-0.002(2)$ & 0.005 & $0.001(3)$ \\
\hline $\mathrm{C} 27$ & 0.039 (3) & $0.049(3)$ & $0.053(4)$ & $-0.003(3)$ & $0.011(3)$ & 0.007 (3) \\
\hline $\mathrm{C} 28$ & $0.033(3)$ & $0.030(3)$ & $0.042(3)$ & $0.000(2)$ & $-0.002(2)$ & $0.000(2)$ \\
\hline $\mathrm{C} 43 \mathrm{~A}$ & $0.067(5)$ & $0.070(6)$ & $0.099(6)$ & $0.003(5)$ & $0.010(5)$ & $-0.003(5)$ \\
\hline $\mathrm{C} 44 \mathrm{~A}$ & $0.062(3)$ & $0.075(4)$ & $0.101(4)$ & 0.005 & $0.010(3)$ & $-0.012(3)$ \\
\hline $\mathrm{C} 45 \mathrm{~A}$ & $0.060(3)$ & $0.075(4)$ & $0.098(4)$ & $0.006(3)$ & $0.008(3)$ & $-0.008(4)$ \\
\hline $\mathrm{C} 46 \mathrm{~A}$ & $0.062(3)$ & $0.074(4)$ & $0.096(4)$ & $0.007(3)$ & $0.012(3)$ & $-0.007(4)$ \\
\hline C47A & $0.061(4)$ & $0.076(4)$ & $0.101(4)$ & 0.005 & $0.011(3)$ & $-0.009(4)$ \\
\hline C48A & $0.062(4)$ & $0.079(4)$ & $0.106(4)$ & $0.005(3)$ & $0.012(4)$ & -0.011 \\
\hline C49A & $0.062(3)$ & 0.077 (4) & $0.104(4)$ & $0.006(3)$ & $0.011(3)$ & $-0.013(4)$ \\
\hline O6A & $0.041(2)$ & $0.030(4)$ & $0.028(2)$ & -0.013 & 0.007 (2) & $-0.003(2)$ \\
\hline C1E & $0.033(2)$ & $0.021(2)$ & 0.034 & $-0.002(2)$ & $-0.001(2)$ & $0.004(2)$ \\
\hline $\mathrm{C} 2 \mathrm{E}$ & $0.034(2)$ & $0.021(2)$ & $0.037(3)$ & $-0.003(2)$ & $0.000(2)$ & $0.001(2)$ \\
\hline $\mathrm{C} 3 \mathrm{E}$ & $0.038(3)$ & $0.023(2)$ & 0.040 & $-0.005(2)$ & $-0.004(2)$ & $-0.003(2)$ \\
\hline $\mathrm{C} 4 \mathrm{E}$ & $0.041(3)$ & $0.024(2)$ & $0.042(3)$ & $0.000(2)$ & -0.005 & $-0.003(2)$ \\
\hline $\mathrm{C} 5 \mathrm{E}$ & $0.040(2)$ & $0.024(2)$ & $0.040(3)$ & $0.005(2)$ & $-0.002(2)$ & $-0.001(2)$ \\
\hline C6E & $0.034(2)$ & $0.021(2)$ & $0.036(2)$ & 0.0045 (19) & $-0.001(2)$ & $0.001(2)$ \\
\hline C7E & $0.037(2)$ & $0.0260(19)$ & $0.036(2)$ & $0.0073(19)$ & $-0.002(2)$ & $-0.0042(19)$ \\
\hline $\mathrm{C} 16 \mathrm{E}$ & 0.053 & $0.050(3)$ & $0.042(3)$ & $0.005(2)$ & $-0.012(2)$ & $-0.008(2)$ \\
\hline $\mathrm{C} 8 \mathrm{E}$ & $0.035(2)$ & $0.027(2)$ & $0.040(2)$ & $0.0075(19)$ & $0.000(2)$ & $-0.007(2)$ \\
\hline C9E & $0.037(3)$ & $0.032(2)$ & $0.044(3)$ & $0.011(2)$ & $0.006(2)$ & $-0.003(2)$ \\
\hline $\mathrm{C} 10 \mathrm{E}$ & $0.040(3)$ & $0.038(3)$ & $0.049(3)$ & $0.010(3)$ & 0.009 & $-0.001(3)$ \\
\hline C11E & $0.040(3)$ & $0.041(3)$ & $0.054(3)$ & 0.009 (3) & 0.015 & $0.001(3)$ \\
\hline C12E & $0.036(2)$ & $0.035(2)$ & 0.055 & $0.006(2)$ & $0.011(2)$ & $-0.002(2)$ \\
\hline C13E & $0.035(2)$ & $0.032(2)$ & $0.049(2)$ & $0.0080(19)$ & $0.004(2)$ & $-0.006(2)$ \\
\hline $\mathrm{C} 14 \mathrm{E}$ & $0.040(3)$ & $0.037(2)$ & $0.047(3)$ & $0.007(2)$ & $-0.003(2)$ & $-0.007(2)$ \\
\hline C15E & $0.042(2)$ & $0.040(2)$ & $0.042(2)$ & $0.007(2)$ & $-0.007(2)$ & $-0.009(2)$ \\
\hline C17E & $0.060(3)$ & $0.056(3)$ & $0.041(3)$ & $0.007(3)$ & $-0.011(3)$ & $-0.004(3)$ \\
\hline $\mathrm{C} 18 \mathrm{E}$ & $0.061(3)$ & $0.054(2)$ & 0.040 & 0.007 (3) & $-0.006(3)$ & $-0.002(2)$ \\
\hline C19E & $0.054(2)$ & $0.046(2)$ & $0.036(2)$ & 0.007 (2) & $-0.007(2)$ & $-0.003(2)$ \\
\hline C20E & $0.043(2)$ & $0.036(2)$ & $0.035(2)$ & $0.006(2)$ & $-0.006(2)$ & $-0.007(2)$ \\
\hline C21A & $0.042(3)$ & $0.032(4)$ & $0.028(2)$ & $-0.011(3)$ & $0.008(2)$ & $-0.002(3)$ \\
\hline $\mathrm{C} 22 \mathrm{~A}$ & $0.046(3)$ & $0.032(4)$ & $0.028(2)$ & -0.011 & $0.007(3)$ & $-0.002(3)$ \\
\hline $\mathrm{C} 23 \mathrm{~A}$ & $0.050(3)$ & $0.033(4)$ & $0.031(2)$ & $-0.012(3)$ & $0.005(2)$ & $-0.001(3)$ \\
\hline $\mathrm{C} 24 \mathrm{~A}$ & $0.045(3)$ & $0.033(3)$ & $0.030(2)$ & $-0.013(3)$ & $0.004(2)$ & $-0.002(2)$ \\
\hline
\end{tabular}




\begin{tabular}{|c|c|c|c|c|c|c|}
\hline C29A & $0.089(5)$ & $0.042(4)$ & $0.032(5)$ & $0.003(4)$ & $0.016(4)$ & $0.004(4)$ \\
\hline $\mathrm{C} 30 \mathrm{~A}$ & $0.083(3)$ & $0.041(2)$ & $0.036(3)$ & $-0.007(2)$ & $0.006(2)$ & $0.000(2)$ \\
\hline $\mathrm{C} 31 \mathrm{~A}$ & $0.081(3)$ & $0.044(3)$ & $0.038(3)$ & $-0.001(3)$ & $0.007(3)$ & $0.000(3)$ \\
\hline $\mathrm{C} 32 \mathrm{~A}$ & $0.079(4)$ & $0.041(3)$ & $0.034(3)$ & $-0.001(3)$ & 0.009 (3) & $0.002(3)$ \\
\hline C33A & 0.077 (4) & $0.037(3)$ & $0.036(3)$ & -0.009 (3) & $0.002(3)$ & $0.004(3)$ \\
\hline $\mathrm{C} 34 \mathrm{~A}$ & 0.077 (4) & $0.039(3)$ & $0.036(3)$ & $-0.010(3)$ & 0.008 & $0.002(3)$ \\
\hline $\mathrm{C} 35 \mathrm{~A}$ & $0.082(3)$ & $0.040(3)$ & $0.034(3)$ & $-0.016(3)$ & 0.009 (3) & $0.000(3)$ \\
\hline O6B & $0.041(2)$ & $0.032(4)$ & $0.028(2)$ & $-0.014(3)$ & $0.007(2)$ & $-0.002(2)$ \\
\hline $\mathrm{C} 5 \mathrm{~F}$ & $0.037(3)$ & $0.022(2)$ & 0.039 (3) & $0.006(2)$ & -0.001 (3) & -0.001 (2) \\
\hline $\mathrm{C} 6 \mathrm{~F}$ & $0.035(2)$ & $0.022(2)$ & $0.037(2)$ & $0.003(2)$ & $0.001(2)$ & $0.001(2)$ \\
\hline $\mathrm{C} 1 \mathrm{~F}$ & 0.035 & $0.021(2)$ & $0.037(3)$ & $-0.001(2)$ & $0.001(2)$ & $0.003(2)$ \\
\hline $\mathrm{C} 2 \mathrm{~F}$ & $0.034(3)$ & $0.022(2)$ & $0.036(3)$ & $-0.002(2)$ & $-0.002(3)$ & $0.001(2)$ \\
\hline $\mathrm{C} 3 \mathrm{~F}$ & 0.038 & $0.025(2)$ & $0.041(3)$ & $-0.003(2)$ & -0.003 & $-0.002(2)$ \\
\hline $\mathrm{C} 4 \mathrm{~F}$ & $0.040(3)$ & $0.024(2)$ & $0.042(3)$ & $-0.001(2)$ & $-0.003(3)$ & $-0.002(2)$ \\
\hline $\mathrm{C} 7 \mathrm{~F}$ & $0.037(2)$ & $0.024(2)$ & $0.035(2)$ & $0.005(2)$ & $-0.002(2)$ & $-0.0050(19)$ \\
\hline $\mathrm{C} 8 \mathrm{~F}$ & $0.046(2)$ & $0.036(2)$ & $0.035(2)$ & 0.009 (2) & $-0.005(2)$ & $-0.006(2)$ \\
\hline C9F & $0.052(3)$ & $0.042(3)$ & $0.035(3)$ & $0.010(3)$ & -0.004 (3) & -0.004 (3) \\
\hline $\mathrm{C} 10 \mathrm{~F}$ & 0.058 & $0.050(3)$ & 0.037 (3) & 0.008 & $-0.005(3)$ & $-0.002(3)$ \\
\hline $\mathrm{C} 11 \mathrm{~F}$ & 0.062 & $0.054(3)$ & $0.040(3)$ & 0.009 (3) & -0.010 & $-0.001(3)$ \\
\hline $\mathrm{C} 12 \mathrm{~F}$ & $0.057(3)$ & $0.053(3)$ & $0.040(3)$ & 0.009 (3) & -0.013 & $-0.004(2)$ \\
\hline $\mathrm{C} 13 \mathrm{~F}$ & $0.052(2)$ & $0.045(2)$ & $0.039(2)$ & $0.008(2)$ & -0.009 (2) & $-0.006(2)$ \\
\hline $\mathrm{C} 14 \mathrm{~F}$ & $0.044(2)$ & $0.040(2)$ & $0.043(2)$ & $0.006(2)$ & $-0.009(2)$ & $-0.008(2)$ \\
\hline $\mathrm{C} 15 \mathrm{~F}$ & $0.038(2)$ & $0.033(2)$ & $0.046(2)$ & $0.009(2)$ & $-0.001(2)$ & $-0.007(2)$ \\
\hline $\mathrm{C} 16 \mathrm{~F}$ & $0.035(2)$ & $0.035(2)$ & $0.053(3)$ & $0.006(2)$ & $0.003(3)$ & $-0.006(2)$ \\
\hline $\mathrm{C} 17 \mathrm{~F}$ & $0.036(3)$ & $0.037(2)$ & 0.055 & $0.007(2)$ & $0.011(2)$ & $-0.004(2)$ \\
\hline $\mathrm{C} 18 \mathrm{~F}$ & $0.038(3)$ & $0.035(2)$ & $0.052(3)$ & $0.006(2)$ & $0.009(2)$ & $-0.004(2)$ \\
\hline $\mathrm{C} 19 \mathrm{~F}$ & $0.035(2)$ & $0.032(2)$ & $0.046(3)$ & $0.006(2)$ & $0.006(2)$ & $-0.007(2)$ \\
\hline $\mathrm{C} 20 \mathrm{~F}$ & $0.035(2)$ & $0.027(2)$ & $0.043(2)$ & 0.0072 (19) & $0.001(2)$ & $-0.006(2)$ \\
\hline C21B & $0.042(3)$ & $0.030(4)$ & $0.028(2)$ & $-0.012(3)$ & $0.008(2)$ & $-0.002(3)$ \\
\hline $\mathrm{C} 22 \mathrm{~B}$ & 0.045 & $0.031(4)$ & $0.029(2)$ & $-0.012(3)$ & 0.007 (3) & $-0.001(3)$ \\
\hline C23B & 0.049 & $0.032(4)$ & $0.031(3)$ & -0.011 & $0.004(2)$ & $0.000(3)$ \\
\hline C24B & 0.045 & $0.032(3)$ & $0.030(2)$ & $-0.013(3)$ & $0.006(2)$ & $-0.002(3)$ \\
\hline $\mathrm{C} 29 \mathrm{~B}$ & $0.085(5)$ & $0.045(4)$ & $0.033(4)$ & -0.003 & $-0.001(4)$ & $0.006(4)$ \\
\hline $\mathrm{C} 30 \mathrm{~B}$ & $0.081(3)$ & $0.041(2)$ & $0.036(2)$ & $-0.007(2)$ & 0.007 (2) & $0.001(2)$ \\
\hline C31B & $0.082(3)$ & 0.045 & 0.040 & $0.000(3)$ & 0.006 & $-0.002(3)$ \\
\hline C32B & $0.089(4)$ & $0.047(3)$ & $0.044(3)$ & $-0.001(3)$ & 0.008 & $-0.001(3)$ \\
\hline C33B & $0.094(4)$ & $0.047(3)$ & $0.042(3)$ & -0.005 & 0.003 & $0.000(3)$ \\
\hline C34B & $0.090(4)$ & $0.046(3)$ & $0.040(3)$ & $-0.015(3)$ & 0.008 & $-0.001(3)$ \\
\hline C35B & $0.084(3)$ & $0.042(3)$ & 0.035 & $-0.014(3)$ & 0.009 (3) & $0.000(3)$ \\
\hline C43B & $0.054(5)$ & $0.081(6)$ & $0.107(6)$ & $0.003(5)$ & $0.009(5)$ & $-0.005(6)$ \\
\hline C44B & $0.059(3)$ & $0.075(4)$ & 0.099 (4) & 0.005 & $0.011(3)$ & $-0.012(3)$ \\
\hline C45B & 0.059 (3) & $0.073(4)$ & 0.095 (4) & $0.004(3)$ & 0.010 & $-0.009(3)$ \\
\hline $\mathrm{C} 46 \mathrm{~B}$ & 0.060 & $0.074(4)$ & 0.098 (4) & 0.005 & 0.008 & -0.009 (4) \\
\hline C47B & $0.061(3)$ & $0.074(4)$ & 0.102 (4) & 0.005 & 0.007 (3) & $-0.013(4)$ \\
\hline C48B & $0.062(3)$ & $0.079(4)$ & $0.106(4)$ & $0.006(3)$ & 0.007 (3) & $-0.014(4)$ \\
\hline C49B & $0.060(3)$ & 0.077 (4) & 0.103 (4) & 0.007 (3) & 0.009 (3) & $-0.016(4)$ \\
\hline $\mathrm{C} 36 \mathrm{~B}$ & $0.046(6)$ & $0.069(7)$ & 0.067 (7) & $-0.018(6)$ & $0.004(6)$ & $0.012(7)$ \\
\hline C37B & $0.042(3)$ & $0.076(4)$ & $0.061(4)$ & $-0.012(3)$ & $0.005(3)$ & $0.009(3)$ \\
\hline
\end{tabular}




\begin{tabular}{lllllll} 
& & & & \\
C38B & $0.040(3)$ & $0.077(4)$ & $0.057(4)$ & $-0.010(3)$ & $0.001(3)$ & $0.010(3)$ \\
C39B & $0.039(4)$ & $0.077(4)$ & $0.056(4)$ & $-0.010(3)$ & $0.000(3)$ & $0.010(3)$ \\
C40B & $0.042(4)$ & $0.078(4)$ & $0.058(4)$ & $-0.012(3)$ & $0.002(3)$ & $0.008(3)$ \\
C41B & $0.044(3)$ & $0.079(4)$ & $0.060(4)$ & $-0.014(3)$ & $0.006(3)$ & $0.006(3)$ \\
C42B & $0.044(3)$ & $0.077(4)$ & $0.061(4)$ & $-0.013(3)$ & $0.007(3)$ & $0.009(3)$ \\
C36A & $0.050(4)$ & $0.089(5)$ & $0.073(5)$ & $-0.015(4)$ & $0.002(4)$ & $-0.005(5)$ \\
C37A & $0.042(3)$ & $0.077(4)$ & $0.059(4)$ & $-0.014(3)$ & $0.007(3)$ & $0.005(3)$ \\
C38A & $0.045(3)$ & $0.077(4)$ & $0.062(4)$ & $-0.015(3)$ & $0.008(3)$ & $0.009(3)$ \\
C39A & $0.043(3)$ & $0.075(4)$ & $0.063(4)$ & $-0.011(3)$ & $0.002(3)$ & $0.011(3)$ \\
C40A & $0.038(3)$ & $0.078(4)$ & $0.056(4)$ & $-0.008(3)$ & $0.002(3)$ & $0.010(3)$ \\
C41A & $0.039(3)$ & $0.078(4)$ & $0.054(4)$ & $-0.009(3)$ & $0.000(3)$ & $0.009(3)$ \\
C42A & $0.041(3)$ & $0.076(4)$ & $0.056(4)$ & $-0.011(3)$ & $0.001(3)$ & $0.008(3)$ \\
\hline
\end{tabular}

Geometric parameters ( $\left.\AA,{ }^{\circ}\right)$

\begin{tabular}{|c|c|c|c|}
\hline $\mathrm{Yb} 1-\mathrm{Yb} 2$ & $3.2587(7)$ & $\mathrm{C} 45 \mathrm{~A}-\mathrm{H} 45 \mathrm{~A}$ & 0.9500 \\
\hline $\mathrm{Yb} 1-\mathrm{O} 1$ & $2.191(3)$ & $\mathrm{C} 45 \mathrm{~A}-\mathrm{C} 46 \mathrm{~A}$ & 1.3894 \\
\hline $\mathrm{Yb} 1-\mathrm{O} 2$ & $2.236(3)$ & $\mathrm{C} 46 \mathrm{~A}-\mathrm{H} 46 \mathrm{~A}$ & 0.9500 \\
\hline $\mathrm{Yb} 1-\mathrm{O} 3$ & $2.077(3)$ & $\mathrm{C} 46 \mathrm{~A}-\mathrm{C} 47 \mathrm{~A}$ & 1.3904 \\
\hline $\mathrm{Yb} 1-\mathrm{O} 5$ & $2.306(3)$ & $\mathrm{C} 47 \mathrm{~A}-\mathrm{H} 47 \mathrm{~A}$ & 0.9500 \\
\hline $\mathrm{Yb} 1-\mathrm{O} 6 \mathrm{~A}$ & $2.309(6)$ & $\mathrm{C} 47 \mathrm{~A}-\mathrm{C} 48 \mathrm{~A}$ & 1.3896 \\
\hline $\mathrm{Yb} 1-\mathrm{O} 7$ & $2.229(3)$ & $\mathrm{C} 48 \mathrm{~A}-\mathrm{H} 48 \mathrm{~A}$ & 0.9500 \\
\hline $\mathrm{Yb} 1-\mathrm{O} 6 \mathrm{~B}$ & $2.310(7)$ & $\mathrm{C} 48 \mathrm{~A}-\mathrm{C} 49 \mathrm{~A}$ & 1.3901 \\
\hline $\mathrm{Yb} 2-\mathrm{O} 1$ & $2.413(3)$ & $\mathrm{C} 49 \mathrm{~A}-\mathrm{H} 49 \mathrm{~A}$ & 0.9500 \\
\hline $\mathrm{Yb} 2-\mathrm{O} 2$ & $2.352(3)$ & $\mathrm{O} 6 \mathrm{~A}-\mathrm{C} 21 \mathrm{~A}$ & $1.42(2)$ \\
\hline $\mathrm{Yb} 2-\mathrm{O} 4$ & $2.165(3)$ & $\mathrm{O} 6 \mathrm{~A}-\mathrm{C} 24 \mathrm{~A}$ & $1.439(18)$ \\
\hline $\mathrm{Yb} 2-\mathrm{O} 7$ & $2.416(3)$ & $\mathrm{C} 1 \mathrm{E}-\mathrm{C} 2 \mathrm{E}$ & 1.3900 \\
\hline $\mathrm{Yb} 2-\mathrm{C} 18 \mathrm{D}$ & $3.190(3)$ & $\mathrm{C} 1 \mathrm{E}-\mathrm{C} 6 \mathrm{E}$ & 1.3900 \\
\hline $\mathrm{Yb} 2-\mathrm{C} 19 \mathrm{D}$ & $2.970(3)$ & $\mathrm{C} 2 \mathrm{E}-\mathrm{H} 2 \mathrm{E}$ & 0.9500 \\
\hline $\mathrm{O} 1-\mathrm{C} 1 \mathrm{D}$ & $1.353(5)$ & $\mathrm{C} 2 \mathrm{E}-\mathrm{C} 3 \mathrm{E}$ & 1.3900 \\
\hline $\mathrm{O} 2-\mathrm{C} 1 \mathrm{~B}$ & $1.357(5)$ & $\mathrm{C} 3 \mathrm{E}-\mathrm{H} 3 \mathrm{E}$ & 0.9500 \\
\hline $\mathrm{O} 3-\mathrm{C} 1 \mathrm{~A}$ & $1.338(5)$ & $\mathrm{C} 3 \mathrm{E}-\mathrm{C} 4 \mathrm{E}$ & 1.3900 \\
\hline $\mathrm{O} 4-\mathrm{C} 1 \mathrm{C}$ & $1.320(5)$ & $\mathrm{C} 4 \mathrm{E}-\mathrm{H} 4 \mathrm{E}$ & 0.9500 \\
\hline $\mathrm{O} 5-\mathrm{C} 25$ & $1.464(5)$ & $\mathrm{C} 4 \mathrm{E}-\mathrm{C} 5 \mathrm{E}$ & 1.3900 \\
\hline $\mathrm{O} 5-\mathrm{C} 28$ & $1.457(6)$ & $\mathrm{C} 5 \mathrm{E}-\mathrm{H} 5 \mathrm{E}$ & 0.9500 \\
\hline $\mathrm{O} 7-\mathrm{C} 1 \mathrm{E}$ & $1.336(5)$ & $\mathrm{C} 5 \mathrm{E}-\mathrm{C} 6 \mathrm{E}$ & 1.3900 \\
\hline $\mathrm{O} 7-\mathrm{C} 1 \mathrm{~F}$ & $1.431(5)$ & $\mathrm{C} 6 \mathrm{E}-\mathrm{C} 7 \mathrm{E}$ & $1.526(5)$ \\
\hline $\mathrm{C} 1 \mathrm{~A}-\mathrm{C} 2 \mathrm{~A}$ & $1.394(6)$ & $\mathrm{C} 7 \mathrm{E}-\mathrm{C} 8 \mathrm{E}$ & 1.4280 \\
\hline $\mathrm{C} 1 \mathrm{~A}-\mathrm{C} 6 \mathrm{~A}$ & $1.414(6)$ & $\mathrm{C} 7 \mathrm{E}-\mathrm{C} 20 \mathrm{E}$ & 1.4132 \\
\hline $\mathrm{C} 1 \mathrm{~B}-\mathrm{C} 2 \mathrm{~B}$ & $1.388(6)$ & $\mathrm{C} 16 \mathrm{E}-\mathrm{H} 16 \mathrm{E}$ & 0.9500 \\
\hline $\mathrm{C} 1 \mathrm{~B}-\mathrm{C} 6 \mathrm{~B}$ & $1.401(6)$ & $\mathrm{C} 16 \mathrm{E}-\mathrm{C} 15 \mathrm{E}$ & 1.4220 \\
\hline $\mathrm{C} 1 \mathrm{C}-\mathrm{C} 2 \mathrm{C}$ & $1.405(7)$ & $\mathrm{C} 16 \mathrm{E}-\mathrm{C} 17 \mathrm{E}$ & 1.3461 \\
\hline $\mathrm{C} 1 \mathrm{C}-\mathrm{C} 6 \mathrm{C}$ & $1.417(7)$ & $\mathrm{C} 8 \mathrm{E}-\mathrm{C} 9 \mathrm{E}$ & 1.4294 \\
\hline $\mathrm{C} 1 \mathrm{D}-\mathrm{C} 2 \mathrm{D}$ & $1.396(7)$ & $\mathrm{C} 8 \mathrm{E}-\mathrm{C} 13 \mathrm{E}$ & 1.4241 \\
\hline $\mathrm{C} 1 \mathrm{D}-\mathrm{C} 6 \mathrm{D}$ & $1.399(7)$ & C9E-H9E & 0.9500 \\
\hline $\mathrm{C} 2 \mathrm{~A}-\mathrm{H} 2 \mathrm{~A}$ & 0.9500 & $\mathrm{C} 9 \mathrm{E}-\mathrm{C} 10 \mathrm{E}$ & 1.3833 \\
\hline $\mathrm{C} 2 \mathrm{~A}-\mathrm{C} 3 \mathrm{~A}$ & $1.375(6)$ & $\mathrm{C} 10 \mathrm{E}-\mathrm{H} 10 \mathrm{E}$ & 0.9500 \\
\hline $\mathrm{C} 2 \mathrm{~B}-\mathrm{H} 2 \mathrm{~B}$ & 0.9500 & $\mathrm{C} 10 \mathrm{E}-\mathrm{C} 11 \mathrm{E}$ & 1.4114 \\
\hline
\end{tabular}




\begin{tabular}{|c|c|c|c|}
\hline $\mathrm{C} 2 \mathrm{~B}-\mathrm{C} 3 \mathrm{~B}$ & $1.373(6)$ & C11E-H11E & 0.9500 \\
\hline $\mathrm{C} 2 \mathrm{C}-\mathrm{H} 2 \mathrm{C}$ & 0.9500 & $\mathrm{C} 11 \mathrm{E}-\mathrm{C} 12 \mathrm{E}$ & 1.3817 \\
\hline $\mathrm{C} 2 \mathrm{C}-\mathrm{C} 3 \mathrm{C}$ & $1.384(7)$ & $\mathrm{C} 12 \mathrm{E}-\mathrm{H} 12 \mathrm{E}$ & 0.9500 \\
\hline $\mathrm{C} 2 \mathrm{D}-\mathrm{H} 2 \mathrm{D}$ & 0.9500 & $\mathrm{C} 12 \mathrm{E}-\mathrm{C} 13 \mathrm{E}$ & 1.4270 \\
\hline $\mathrm{C} 2 \mathrm{D}-\mathrm{C} 3 \mathrm{D}$ & $1.382(7)$ & $\mathrm{C} 13 \mathrm{E}-\mathrm{C} 14 \mathrm{E}$ & 1.3925 \\
\hline $\mathrm{C} 3 \mathrm{~A}-\mathrm{H} 3 \mathrm{~A}$ & 0.9500 & $\mathrm{C} 14 \mathrm{E}-\mathrm{H} 14 \mathrm{E}$ & 0.9500 \\
\hline $\mathrm{C} 3 \mathrm{~A}-\mathrm{C} 4 \mathrm{~A}$ & $1.368(7)$ & $\mathrm{C} 14 \mathrm{E}-\mathrm{C} 15 \mathrm{E}$ & 1.3868 \\
\hline $\mathrm{C} 3 \mathrm{~B}-\mathrm{H} 3 \mathrm{~B}$ & 0.9500 & $\mathrm{C} 15 \mathrm{E}-\mathrm{C} 20 \mathrm{E}$ & 1.4215 \\
\hline $\mathrm{C} 3 \mathrm{~B}-\mathrm{C} 4 \mathrm{~B}$ & $1.381(7)$ & $\mathrm{C} 17 \mathrm{E}-\mathrm{H} 17 \mathrm{E}$ & 0.9500 \\
\hline $\mathrm{C} 3 \mathrm{C}-\mathrm{H} 3 \mathrm{C}$ & 0.9500 & $\mathrm{C} 17 \mathrm{E}-\mathrm{C} 18 \mathrm{E}$ & 1.4117 \\
\hline $\mathrm{C} 3 \mathrm{C}-\mathrm{C} 4 \mathrm{C}$ & $1.380(8)$ & $\mathrm{C} 18 \mathrm{E}-\mathrm{H} 18 \mathrm{E}$ & 0.9500 \\
\hline $\mathrm{C} 3 \mathrm{D}-\mathrm{H} 3 \mathrm{D}$ & 0.9500 & $\mathrm{C} 18 \mathrm{E}-\mathrm{C} 19 \mathrm{E}$ & 1.3440 \\
\hline $\mathrm{C} 3 \mathrm{D}-\mathrm{C} 4 \mathrm{D}$ & $1.375(8)$ & C19E-H19E & 0.9500 \\
\hline $\mathrm{C} 4 \mathrm{~A}-\mathrm{H} 4 \mathrm{~A}$ & 0.9500 & $\mathrm{C} 19 \mathrm{E}-\mathrm{C} 20 \mathrm{E}$ & 1.4494 \\
\hline $\mathrm{C} 4 \mathrm{~A}-\mathrm{C} 5 \mathrm{~A}$ & $1.380(7)$ & $\mathrm{C} 21 \mathrm{~A}-\mathrm{H} 21 \mathrm{~A}$ & 0.9900 \\
\hline $\mathrm{C} 4 \mathrm{~B}-\mathrm{H} 4 \mathrm{~B}$ & 0.9500 & $\mathrm{C} 21 \mathrm{~A}-\mathrm{H} 21 \mathrm{~B}$ & 0.9900 \\
\hline $\mathrm{C} 4 \mathrm{~B}-\mathrm{C} 5 \mathrm{~B}$ & $1.381(7)$ & $\mathrm{C} 21 \mathrm{~A}-\mathrm{C} 22 \mathrm{~A}$ & $1.56(2)$ \\
\hline $\mathrm{C} 4 \mathrm{C}-\mathrm{H} 4 \mathrm{C}$ & 0.9500 & $\mathrm{C} 22 \mathrm{~A}-\mathrm{H} 22 \mathrm{~A}$ & 0.9900 \\
\hline $\mathrm{C} 4 \mathrm{C}-\mathrm{C} 5 \mathrm{C}$ & $1.385(8)$ & $\mathrm{C} 22 \mathrm{~A}-\mathrm{H} 22 \mathrm{~B}$ & 0.9900 \\
\hline $\mathrm{C} 4 \mathrm{D}-\mathrm{H} 4 \mathrm{D}$ & 0.9500 & $\mathrm{C} 22 \mathrm{~A}-\mathrm{C} 23 \mathrm{~A}$ & $1.40(2)$ \\
\hline $\mathrm{C} 4 \mathrm{D}-\mathrm{C} 5 \mathrm{D}$ & $1.382(8)$ & $\mathrm{C} 23 \mathrm{~A}-\mathrm{H} 23 \mathrm{~A}$ & 0.9900 \\
\hline $\mathrm{C} 5 \mathrm{~A}-\mathrm{H} 5 \mathrm{~A}$ & 0.9500 & $\mathrm{C} 23 \mathrm{~A}-\mathrm{H} 23 \mathrm{~B}$ & 0.9900 \\
\hline $\mathrm{C} 5 \mathrm{~A}-\mathrm{C} 6 \mathrm{~A}$ & $1.394(6)$ & $\mathrm{C} 23 \mathrm{~A}-\mathrm{C} 24 \mathrm{~A}$ & $1.381(17)$ \\
\hline $\mathrm{C} 5 \mathrm{~B}-\mathrm{H} 5 \mathrm{~B}$ & 0.9500 & $\mathrm{C} 24 \mathrm{~A}-\mathrm{H} 24 \mathrm{~A}$ & 0.9900 \\
\hline $\mathrm{C} 5 \mathrm{~B}-\mathrm{C} 6 \mathrm{~B}$ & $1.385(7)$ & $\mathrm{C} 24 \mathrm{~A}-\mathrm{H} 24 \mathrm{~B}$ & 0.9900 \\
\hline $\mathrm{C} 5 \mathrm{C}-\mathrm{H} 5 \mathrm{C}$ & 0.9500 & $\mathrm{C} 29 \mathrm{~A}-\mathrm{H} 29 \mathrm{~A}$ & 0.9800 \\
\hline $\mathrm{C} 5 \mathrm{C}-\mathrm{C} 6 \mathrm{C}$ & $1.384(6)$ & $\mathrm{C} 29 \mathrm{~A}-\mathrm{H} 29 \mathrm{~B}$ & 0.9800 \\
\hline $\mathrm{C} 5 \mathrm{D}-\mathrm{H} 5 \mathrm{D}$ & 0.9500 & $\mathrm{C} 29 \mathrm{~A}-\mathrm{H} 29 \mathrm{C}$ & 0.9800 \\
\hline $\mathrm{C} 5 \mathrm{D}-\mathrm{C} 6 \mathrm{D}$ & $1.399(7)$ & $\mathrm{C} 29 \mathrm{~A}-\mathrm{C} 30 \mathrm{~A}$ & 1.4201 \\
\hline $\mathrm{C} 6 \mathrm{~A}-\mathrm{C} 7 \mathrm{~A}$ & $1.481(6)$ & $\mathrm{C} 30 \mathrm{~A}-\mathrm{C} 31 \mathrm{~A}$ & 1.3905 \\
\hline $\mathrm{C} 6 \mathrm{~B}-\mathrm{C} 7 \mathrm{~B}$ & $1.496(6)$ & $\mathrm{C} 30 \mathrm{~A}-\mathrm{C} 35 \mathrm{~A}$ & 1.3887 \\
\hline $\mathrm{C} 6 \mathrm{C}-\mathrm{C} 7 \mathrm{C}$ & $1.489(7)$ & $\mathrm{C} 31 \mathrm{~A}-\mathrm{H} 31 \mathrm{~A}$ & 0.9500 \\
\hline $\mathrm{C} 6 \mathrm{D}-\mathrm{C} 7 \mathrm{D}$ & $1.493(7)$ & $\mathrm{C} 31 \mathrm{~A}-\mathrm{C} 32 \mathrm{~A}$ & 1.3909 \\
\hline $\mathrm{C} 7 \mathrm{~A}-\mathrm{C} 8 \mathrm{~A}$ & $1.409(6)$ & $\mathrm{C} 32 \mathrm{~A}-\mathrm{H} 32 \mathrm{~A}$ & 0.9500 \\
\hline $\mathrm{C} 7 \mathrm{~A}-\mathrm{C} 20 \mathrm{~A}$ & $1.416(6)$ & $\mathrm{C} 32 \mathrm{~A}-\mathrm{C} 33 \mathrm{~A}$ & 1.3908 \\
\hline $\mathrm{C} 7 \mathrm{~B}-\mathrm{C} 8 \mathrm{~B}$ & $1.407(7)$ & $\mathrm{C} 33 \mathrm{~A}-\mathrm{H} 33 \mathrm{~A}$ & 0.9500 \\
\hline $\mathrm{C} 7 \mathrm{~B}-\mathrm{C} 20 \mathrm{~B}$ & $1.408(7)$ & $\mathrm{C} 33 \mathrm{~A}-\mathrm{C} 34 \mathrm{~A}$ & 1.3893 \\
\hline $\mathrm{C} 7 \mathrm{C}-\mathrm{C} 8 \mathrm{C}$ & $1.416(7)$ & $\mathrm{C} 34 \mathrm{~A}-\mathrm{H} 34 \mathrm{~A}$ & 0.9500 \\
\hline $\mathrm{C} 7 \mathrm{C}-\mathrm{C} 20 \mathrm{C}$ & $1.415(6)$ & $\mathrm{C} 34 \mathrm{~A}-\mathrm{C} 35 \mathrm{~A}$ & 1.3906 \\
\hline $\mathrm{C} 7 \mathrm{D}-\mathrm{C} 8 \mathrm{D}$ & $1.420(7)$ & $\mathrm{C} 35 \mathrm{~A}-\mathrm{H} 35 \mathrm{~A}$ & 0.9500 \\
\hline $\mathrm{C} 7 \mathrm{D}-\mathrm{C} 20 \mathrm{D}$ & $1.414(7)$ & $\mathrm{O} 6 \mathrm{~B}-\mathrm{C} 21 \mathrm{~B}$ & $1.44(3)$ \\
\hline $\mathrm{C} 8 \mathrm{~A}-\mathrm{C} 9 \mathrm{~A}$ & $1.430(6)$ & $\mathrm{O} 6 \mathrm{~B}-\mathrm{C} 24 \mathrm{~B}$ & $1.49(2)$ \\
\hline $\mathrm{C} 8 \mathrm{~A}-\mathrm{C} 13 \mathrm{~A}$ & $1.432(6)$ & $\mathrm{C} 5 \mathrm{~F}-\mathrm{H} 5 \mathrm{~F}$ & 0.9500 \\
\hline $\mathrm{C} 8 \mathrm{~B}-\mathrm{C} 9 \mathrm{~B}$ & $1.430(7)$ & $\mathrm{C} 5 \mathrm{~F}-\mathrm{C} 6 \mathrm{~F}$ & 1.3900 \\
\hline $\mathrm{C} 8 \mathrm{~B}-\mathrm{C} 13 \mathrm{~B}$ & $1.446(6)$ & $\mathrm{C} 5 \mathrm{~F}-\mathrm{C} 4 \mathrm{~F}$ & 1.3900 \\
\hline $\mathrm{C} 8 \mathrm{C}-\mathrm{C} 9 \mathrm{C}$ & $1.431(7)$ & $\mathrm{C} 6 \mathrm{~F}-\mathrm{C} 1 \mathrm{~F}$ & 1.3900 \\
\hline $\mathrm{C} 8 \mathrm{C}-\mathrm{C} 13 \mathrm{C}$ & $1.441(8)$ & $\mathrm{C} 6 \mathrm{~F}-\mathrm{C} 7 \mathrm{~F}$ & $1.490(5)$ \\
\hline C8D-C9D & $1.421(9)$ & $\mathrm{C} 1 \mathrm{~F}-\mathrm{C} 2 \mathrm{~F}$ & 1.3900 \\
\hline
\end{tabular}




\begin{tabular}{|c|c|c|c|}
\hline $\mathrm{C} 8 \mathrm{D}-\mathrm{C} 13 \mathrm{D}$ & $1.420(8)$ & $\mathrm{C} 2 \mathrm{~F}-\mathrm{H} 2 \mathrm{~F}$ & 0.9500 \\
\hline C9A-H9A & 0.9500 & $\mathrm{C} 2 \mathrm{~F}-\mathrm{C} 3 \mathrm{~F}$ & 1.3900 \\
\hline $\mathrm{C} 9 \mathrm{~A}-\mathrm{C} 10 \mathrm{~A}$ & $1.352(7)$ & $\mathrm{C} 3 \mathrm{~F}-\mathrm{H} 3 \mathrm{~F}$ & 0.9500 \\
\hline C9B-H9B & 0.9500 & $\mathrm{C} 3 \mathrm{~F}-\mathrm{C} 4 \mathrm{~F}$ & 1.3900 \\
\hline $\mathrm{C} 9 \mathrm{~B}-\mathrm{C} 10 \mathrm{~B}$ & $1.353(7)$ & $\mathrm{C} 4 \mathrm{~F}-\mathrm{H} 4 \mathrm{~F}$ & 0.9500 \\
\hline $\mathrm{C} 9 \mathrm{C}-\mathrm{H} 9 \mathrm{C}$ & 0.9500 & $\mathrm{C} 7 \mathrm{~F}-\mathrm{C} 8 \mathrm{~F}$ & 1.4125 \\
\hline $\mathrm{C} 9 \mathrm{C}-\mathrm{C} 10 \mathrm{C}$ & $1.380(9)$ & $\mathrm{C} 7 \mathrm{~F}-\mathrm{C} 20 \mathrm{~F}$ & 1.4284 \\
\hline C9D-H9D & 0.9500 & $\mathrm{C} 8 \mathrm{~F}-\mathrm{C} 9 \mathrm{~F}$ & 1.4501 \\
\hline C9D-C10D & $1.356(8)$ & $\mathrm{C} 8 \mathrm{~F}-\mathrm{C} 13 \mathrm{~F}$ & 1.4210 \\
\hline $\mathrm{C} 10 \mathrm{~A}-\mathrm{H} 10 \mathrm{~A}$ & 0.9500 & $\mathrm{C} 9 \mathrm{~F}-\mathrm{H} 9 \mathrm{~F}$ & 0.9500 \\
\hline $\mathrm{C} 10 \mathrm{~A}-\mathrm{C} 11 \mathrm{~A}$ & $1.405(7)$ & $\mathrm{C} 9 \mathrm{~F}-\mathrm{C} 10 \mathrm{~F}$ & 1.3440 \\
\hline $\mathrm{C} 10 \mathrm{~B}-\mathrm{H} 10 \mathrm{~B}$ & 0.9500 & $\mathrm{C} 10 \mathrm{~F}-\mathrm{H} 10 \mathrm{~F}$ & 0.9500 \\
\hline $\mathrm{C} 10 \mathrm{~B}-\mathrm{C} 11 \mathrm{~B}$ & $1.420(7)$ & $\mathrm{C} 10 \mathrm{~F}-\mathrm{C} 11 \mathrm{~F}$ & 1.4120 \\
\hline $\mathrm{C} 10 \mathrm{C}-\mathrm{H} 10 \mathrm{C}$ & 0.9500 & $\mathrm{C} 11 \mathrm{~F}-\mathrm{H} 11 \mathrm{~F}$ & 0.9500 \\
\hline $\mathrm{C} 10 \mathrm{C}-\mathrm{C} 11 \mathrm{C}$ & $1.406(11)$ & $\mathrm{C} 11 \mathrm{~F}-\mathrm{C} 12 \mathrm{~F}$ & 1.3448 \\
\hline C10D-H10D & 0.9500 & $\mathrm{C} 12 \mathrm{~F}-\mathrm{H} 12 \mathrm{~F}$ & 0.9500 \\
\hline $\mathrm{C} 10 \mathrm{D}-\mathrm{C} 11 \mathrm{D}$ & $1.395(11)$ & $\mathrm{C} 12 \mathrm{~F}-\mathrm{C} 13 \mathrm{~F}$ & 1.4220 \\
\hline $\mathrm{C} 11 \mathrm{~A}-\mathrm{H} 11 \mathrm{~A}$ & 0.9500 & $\mathrm{C} 13 \mathrm{~F}-\mathrm{C} 14 \mathrm{~F}$ & 1.3877 \\
\hline $\mathrm{C} 11 \mathrm{~A}-\mathrm{C} 12 \mathrm{~A}$ & $1.359(7)$ & $\mathrm{C} 14 \mathrm{~F}-\mathrm{H} 14 \mathrm{~F}$ & 0.9500 \\
\hline $\mathrm{C} 11 \mathrm{~B}-\mathrm{H} 11 \mathrm{~B}$ & 0.9500 & $\mathrm{C} 14 \mathrm{~F}-\mathrm{C} 15 \mathrm{~F}$ & 1.3922 \\
\hline $\mathrm{C} 11 \mathrm{~B}-\mathrm{C} 12 \mathrm{~B}$ & $1.352(8)$ & $\mathrm{C} 15 \mathrm{~F}-\mathrm{C} 16 \mathrm{~F}$ & 1.4273 \\
\hline $\mathrm{C} 11 \mathrm{C}-\mathrm{H} 11 \mathrm{C}$ & 0.9500 & $\mathrm{C} 15 \mathrm{~F}-\mathrm{C} 20 \mathrm{~F}$ & 1.4232 \\
\hline $\mathrm{C} 11 \mathrm{C}-\mathrm{C} 12 \mathrm{C}$ & $1.329(10)$ & $\mathrm{C} 16 \mathrm{~F}-\mathrm{H} 16 \mathrm{~F}$ & 0.9500 \\
\hline C11D-H11D & 0.9500 & $\mathrm{C} 16 \mathrm{~F}-\mathrm{C} 17 \mathrm{~F}$ & 1.3829 \\
\hline $\mathrm{C} 11 \mathrm{D}-\mathrm{C} 12 \mathrm{D}$ & $1.339(11)$ & $\mathrm{C} 17 \mathrm{~F}-\mathrm{H} 17 \mathrm{~F}$ & 0.9500 \\
\hline $\mathrm{C} 12 \mathrm{~A}-\mathrm{H} 12 \mathrm{~A}$ & 0.9500 & $\mathrm{C} 17 \mathrm{~F}-\mathrm{C} 18 \mathrm{~F}$ & 1.4120 \\
\hline $\mathrm{C} 12 \mathrm{~A}-\mathrm{C} 13 \mathrm{~A}$ & $1.413(7)$ & $\mathrm{C} 18 \mathrm{~F}-\mathrm{H} 18 \mathrm{~F}$ & 0.9500 \\
\hline $\mathrm{C} 12 \mathrm{~B}-\mathrm{H} 12 \mathrm{~B}$ & 0.9500 & $\mathrm{C} 18 \mathrm{~F}-\mathrm{C} 19 \mathrm{~F}$ & 1.3834 \\
\hline $\mathrm{C} 12 \mathrm{~B}-\mathrm{C} 13 \mathrm{~B}$ & $1.433(7)$ & $\mathrm{C} 19 \mathrm{~F}-\mathrm{H} 19 \mathrm{~F}$ & 0.9500 \\
\hline $\mathrm{C} 12 \mathrm{C}-\mathrm{H} 12 \mathrm{C}$ & 0.9500 & $\mathrm{C} 19 \mathrm{~F}-\mathrm{C} 20 \mathrm{~F}$ & 1.4290 \\
\hline $\mathrm{C} 12 \mathrm{C}-\mathrm{C} 13 \mathrm{C}$ & $1.437(8)$ & $\mathrm{C} 21 \mathrm{~B}-\mathrm{H} 21 \mathrm{C}$ & 0.9900 \\
\hline $\mathrm{C} 12 \mathrm{D}-\mathrm{H} 12 \mathrm{D}$ & 0.9500 & $\mathrm{C} 21 \mathrm{~B}-\mathrm{H} 21 \mathrm{D}$ & 0.9900 \\
\hline $\mathrm{C} 12 \mathrm{D}-\mathrm{C} 13 \mathrm{D}$ & $1.443(8)$ & $\mathrm{C} 21 \mathrm{~B}-\mathrm{C} 22 \mathrm{~B}$ & $1.49(3)$ \\
\hline $\mathrm{C} 13 \mathrm{~A}-\mathrm{C} 14 \mathrm{~A}$ & $1.401(6)$ & $\mathrm{C} 22 \mathrm{~B}-\mathrm{H} 22 \mathrm{C}$ & 0.9900 \\
\hline $\mathrm{C} 13 \mathrm{~B}-\mathrm{C} 14 \mathrm{~B}$ & $1.379(7)$ & $\mathrm{C} 22 \mathrm{~B}-\mathrm{H} 22 \mathrm{D}$ & 0.9900 \\
\hline $\mathrm{C} 13 \mathrm{C}-\mathrm{C} 14 \mathrm{C}$ & $1.374(8)$ & $\mathrm{C} 22 \mathrm{~B}-\mathrm{C} 23 \mathrm{~B}$ & $1.63(2)$ \\
\hline $\mathrm{C} 13 \mathrm{D}-\mathrm{C} 14 \mathrm{D}$ & $1.394(9)$ & $\mathrm{C} 23 \mathrm{~B}-\mathrm{H} 23 \mathrm{C}$ & 0.9900 \\
\hline $\mathrm{C} 14 \mathrm{~A}-\mathrm{H} 14 \mathrm{~A}$ & 0.9500 & $\mathrm{C} 23 \mathrm{~B}-\mathrm{H} 23 \mathrm{D}$ & 0.9900 \\
\hline $\mathrm{C} 14 \mathrm{~A}-\mathrm{C} 15 \mathrm{~A}$ & $1.388(7)$ & $\mathrm{C} 23 \mathrm{~B}-\mathrm{C} 24 \mathrm{~B}$ & $1.33(2)$ \\
\hline $\mathrm{C} 14 \mathrm{~B}-\mathrm{H} 14 \mathrm{~B}$ & 0.9500 & $\mathrm{C} 24 \mathrm{~B}-\mathrm{H} 24 \mathrm{C}$ & 0.9900 \\
\hline $\mathrm{C} 14 \mathrm{~B}-\mathrm{C} 15 \mathrm{~B}$ & $1.388(7)$ & $\mathrm{C} 24 \mathrm{~B}-\mathrm{H} 24 \mathrm{D}$ & 0.9900 \\
\hline $\mathrm{C} 14 \mathrm{C}-\mathrm{H} 14 \mathrm{C}$ & 0.9500 & $\mathrm{C} 29 \mathrm{~B}-\mathrm{H} 29 \mathrm{D}$ & 0.9800 \\
\hline $\mathrm{C} 14 \mathrm{C}-\mathrm{C} 15 \mathrm{C}$ & $1.378(7)$ & C29B-H29E & 0.9800 \\
\hline $\mathrm{C} 14 \mathrm{D}-\mathrm{H} 14 \mathrm{D}$ & 0.9500 & $\mathrm{C} 29 \mathrm{~B}-\mathrm{H} 29 \mathrm{~F}$ & 0.9800 \\
\hline $\mathrm{C} 14 \mathrm{D}-\mathrm{C} 15 \mathrm{D}$ & $1.403(8)$ & $\mathrm{C} 29 \mathrm{~B}-\mathrm{C} 30 \mathrm{~B}$ & 1.4186 \\
\hline $\mathrm{C} 15 \mathrm{~A}-\mathrm{C} 16 \mathrm{~A}$ & $1.422(6)$ & $\mathrm{C} 30 \mathrm{~B}-\mathrm{C} 31 \mathrm{~B}$ & 1.3922 \\
\hline $\mathrm{C} 15 \mathrm{~A}-\mathrm{C} 20 \mathrm{~A}$ & $1.438(6)$ & $\mathrm{C} 30 \mathrm{~B}-\mathrm{C} 35 \mathrm{~B}$ & 1.3897 \\
\hline $\mathrm{C} 15 \mathrm{~B}-\mathrm{C} 16 \mathrm{~B}$ & $1.436(7)$ & $\mathrm{C} 31 \mathrm{~B}-\mathrm{H} 31 \mathrm{~B}$ & 0.9500 \\
\hline
\end{tabular}




\begin{tabular}{|c|c|c|c|}
\hline $\mathrm{C} 15 \mathrm{~B}-\mathrm{C} 20 \mathrm{~B}$ & $1.432(6)$ & $\mathrm{C} 31 \mathrm{~B}-\mathrm{C} 32 \mathrm{~B}$ & 1.3893 \\
\hline $\mathrm{C} 15 \mathrm{C}-\mathrm{C} 16 \mathrm{C}$ & $1.434(7)$ & $\mathrm{C} 32 \mathrm{~B}-\mathrm{H} 32 \mathrm{~B}$ & 0.9500 \\
\hline $\mathrm{C} 15 \mathrm{C}-\mathrm{C} 20 \mathrm{C}$ & $1.434(7)$ & $\mathrm{C} 32 \mathrm{~B}-\mathrm{C} 33 \mathrm{~B}$ & 1.3898 \\
\hline $\mathrm{C} 15 \mathrm{D}-\mathrm{C} 16 \mathrm{D}$ & $1.414(8)$ & $\mathrm{C} 33 \mathrm{~B}-\mathrm{H} 33 \mathrm{~B}$ & 0.9500 \\
\hline $\mathrm{C} 15 \mathrm{D}-\mathrm{C} 20 \mathrm{D}$ & $1.426(7)$ & $\mathrm{C} 33 \mathrm{~B}-\mathrm{C} 34 \mathrm{~B}$ & 1.3904 \\
\hline $\mathrm{C} 16 \mathrm{~A}-\mathrm{H} 16 \mathrm{~A}$ & 0.9500 & $\mathrm{C} 34 \mathrm{~B}-\mathrm{H} 34 \mathrm{~B}$ & 0.9500 \\
\hline $\mathrm{C} 16 \mathrm{~A}-\mathrm{C} 17 \mathrm{~A}$ & $1.353(7)$ & $\mathrm{C} 34 \mathrm{~B}-\mathrm{C} 35 \mathrm{~B}$ & 1.3896 \\
\hline $\mathrm{C} 16 \mathrm{~B}-\mathrm{H} 16 \mathrm{~B}$ & 0.9500 & $\mathrm{C} 35 \mathrm{~B}-\mathrm{H} 35 \mathrm{~B}$ & 0.9500 \\
\hline $\mathrm{C} 16 \mathrm{~B}-\mathrm{C} 17 \mathrm{~B}$ & $1.349(8)$ & $\mathrm{C} 43 \mathrm{~B}-\mathrm{H} 43 \mathrm{D}$ & 0.9800 \\
\hline $\mathrm{C} 16 \mathrm{C}-\mathrm{H} 16 \mathrm{C}$ & 0.9500 & $\mathrm{C} 43 \mathrm{~B}-\mathrm{H} 43 \mathrm{E}$ & 0.9800 \\
\hline $\mathrm{C} 16 \mathrm{C}-\mathrm{C} 17 \mathrm{C}$ & $1.350(8)$ & $\mathrm{C} 43 \mathrm{~B}-\mathrm{H} 43 \mathrm{~F}$ & 0.9800 \\
\hline $\mathrm{C} 16 \mathrm{D}-\mathrm{H} 16 \mathrm{D}$ & 0.9500 & $\mathrm{C} 43 \mathrm{~B}-\mathrm{C} 44 \mathrm{~B}$ & 1.4189 \\
\hline $\mathrm{C} 16 \mathrm{D}-\mathrm{C} 17 \mathrm{D}$ & $1.364(8)$ & $\mathrm{C} 44 \mathrm{~B}-\mathrm{C} 45 \mathrm{~B}$ & 1.3923 \\
\hline $\mathrm{C} 17 \mathrm{~A}-\mathrm{H} 17 \mathrm{~A}$ & 0.9500 & $\mathrm{C} 44 \mathrm{~B}-\mathrm{C} 49 \mathrm{~B}$ & 1.3889 \\
\hline $\mathrm{C} 17 \mathrm{~A}-\mathrm{C} 18 \mathrm{~A}$ & $1.415(7)$ & $\mathrm{C} 45 \mathrm{~B}-\mathrm{H} 45 \mathrm{~B}$ & 0.9500 \\
\hline $\mathrm{C} 17 \mathrm{~B}-\mathrm{H} 17 \mathrm{~B}$ & 0.9500 & $\mathrm{C} 45 \mathrm{~B}-\mathrm{C} 46 \mathrm{~B}$ & 1.3896 \\
\hline $\mathrm{C} 17 \mathrm{~B}-\mathrm{C} 18 \mathrm{~B}$ & $1.419(7)$ & $\mathrm{C} 46 \mathrm{~B}-\mathrm{H} 46 \mathrm{~B}$ & 0.9500 \\
\hline $\mathrm{C} 17 \mathrm{C}-\mathrm{H} 17 \mathrm{C}$ & 0.9500 & $\mathrm{C} 46 \mathrm{~B}-\mathrm{C} 47 \mathrm{~B}$ & 1.3906 \\
\hline $\mathrm{C} 17 \mathrm{C}-\mathrm{C} 18 \mathrm{C}$ & $1.421(8)$ & $\mathrm{C} 47 \mathrm{~B}-\mathrm{H} 47 \mathrm{~B}$ & 0.9500 \\
\hline C17D-H17D & 0.9500 & $\mathrm{C} 47 \mathrm{~B}-\mathrm{C} 48 \mathrm{~B}$ & 1.3899 \\
\hline $\mathrm{C} 17 \mathrm{D}-\mathrm{C} 18 \mathrm{D}$ & $1.433(7)$ & $\mathrm{C} 48 \mathrm{~B}-\mathrm{H} 48 \mathrm{~B}$ & 0.9500 \\
\hline $\mathrm{C} 18 \mathrm{~A}-\mathrm{H} 18 \mathrm{~A}$ & 0.9500 & $\mathrm{C} 48 \mathrm{~B}-\mathrm{C} 49 \mathrm{~B}$ & 1.3907 \\
\hline $\mathrm{C} 18 \mathrm{~A}-\mathrm{C} 19 \mathrm{~A}$ & $1.367(6)$ & $\mathrm{C} 49 \mathrm{~B}-\mathrm{H} 49 \mathrm{~B}$ & 0.9500 \\
\hline $\mathrm{C} 18 \mathrm{~B}-\mathrm{H} 18 \mathrm{~B}$ & 0.9500 & $\mathrm{C} 36 \mathrm{~B}-\mathrm{H} 36 \mathrm{~A}$ & 0.9800 \\
\hline $\mathrm{C} 18 \mathrm{~B}-\mathrm{C} 19 \mathrm{~B}$ & $1.366(7)$ & $\mathrm{C} 36 \mathrm{~B}-\mathrm{H} 36 \mathrm{~B}$ & 0.9800 \\
\hline $\mathrm{C} 18 \mathrm{C}-\mathrm{H} 18 \mathrm{C}$ & 0.9500 & $\mathrm{C} 36 \mathrm{~B}-\mathrm{H} 36 \mathrm{C}$ & 0.9800 \\
\hline $\mathrm{C} 18 \mathrm{C}-\mathrm{C} 19 \mathrm{C}$ & $1.349(7)$ & $\mathrm{C} 36 \mathrm{~B}-\mathrm{C} 37 \mathrm{~B}$ & 1.4191 \\
\hline $\mathrm{C} 18 \mathrm{D}-\mathrm{H} 18 \mathrm{D}$ & 0.9500 & $\mathrm{C} 37 \mathrm{~B}-\mathrm{C} 38 \mathrm{~B}$ & 1.3924 \\
\hline $\mathrm{C} 18 \mathrm{D}-\mathrm{C} 19 \mathrm{D}$ & $1.358(7)$ & $\mathrm{C} 37 \mathrm{~B}-\mathrm{C} 42 \mathrm{~B}$ & 1.3880 \\
\hline C19A-H19A & 0.9500 & $\mathrm{C} 38 \mathrm{~B}-\mathrm{H} 38 \mathrm{~B}$ & 0.9500 \\
\hline $\mathrm{C} 19 \mathrm{~A}-\mathrm{C} 20 \mathrm{~A}$ & $1.424(6)$ & $\mathrm{C} 38 \mathrm{~B}-\mathrm{C} 39 \mathrm{~B}$ & 1.3890 \\
\hline $\mathrm{C} 19 \mathrm{~B}-\mathrm{H} 19 \mathrm{~B}$ & 0.9500 & C39B-H39B & 0.9500 \\
\hline $\mathrm{C} 19 \mathrm{~B}-\mathrm{C} 20 \mathrm{~B}$ & $1.425(7)$ & $\mathrm{C} 39 \mathrm{~B}-\mathrm{C} 40 \mathrm{~B}$ & 1.3911 \\
\hline $\mathrm{C} 19 \mathrm{C}-\mathrm{H} 19 \mathrm{C}$ & 0.9500 & $\mathrm{C} 40 \mathrm{~B}-\mathrm{H} 40 \mathrm{~B}$ & 0.9500 \\
\hline $\mathrm{C} 19 \mathrm{C}-\mathrm{C} 20 \mathrm{C}$ & $1.435(7)$ & $\mathrm{C} 40 \mathrm{~B}-\mathrm{C} 41 \mathrm{~B}$ & 1.3898 \\
\hline C19D-H19D & 0.9500 & $\mathrm{C} 41 \mathrm{~B}-\mathrm{H} 41 \mathrm{~B}$ & 0.9500 \\
\hline $\mathrm{C} 19 \mathrm{D}-\mathrm{C} 20 \mathrm{D}$ & $1.430(7)$ & $\mathrm{C} 41 \mathrm{~B}-\mathrm{C} 42 \mathrm{~B}$ & 1.3902 \\
\hline $\mathrm{C} 25-\mathrm{H} 25 \mathrm{~A}$ & 0.9900 & $\mathrm{C} 42 \mathrm{~B}-\mathrm{H} 42 \mathrm{~B}$ & 0.9500 \\
\hline $\mathrm{C} 25-\mathrm{H} 25 \mathrm{~B}$ & 0.9900 & $\mathrm{C} 36 \mathrm{~A}-\mathrm{H} 36 \mathrm{D}$ & 0.9800 \\
\hline $\mathrm{C} 25-\mathrm{C} 26$ & $1.466(8)$ & $\mathrm{C} 36 \mathrm{~A}-\mathrm{H} 36 \mathrm{E}$ & 0.9800 \\
\hline $\mathrm{C} 26-\mathrm{H} 26 \mathrm{~A}$ & 0.9900 & $\mathrm{C} 36 \mathrm{~A}-\mathrm{H} 36 \mathrm{~F}$ & 0.9800 \\
\hline $\mathrm{C} 26-\mathrm{H} 26 \mathrm{~B}$ & 0.9900 & $\mathrm{C} 36 \mathrm{~A}-\mathrm{C} 37 \mathrm{~A}$ & 1.4192 \\
\hline $\mathrm{C} 26-\mathrm{C} 27$ & $1.511(8)$ & $\mathrm{C} 37 \mathrm{~A}-\mathrm{C} 38 \mathrm{~A}$ & 1.3922 \\
\hline $\mathrm{C} 27-\mathrm{H} 27 \mathrm{~A}$ & 0.9900 & $\mathrm{C} 37 \mathrm{~A}-\mathrm{C} 42 \mathrm{~A}$ & 1.3883 \\
\hline $\mathrm{C} 27-\mathrm{H} 27 \mathrm{~B}$ & 0.9900 & C38A-H38A & 0.9500 \\
\hline $\mathrm{C} 27-\mathrm{C} 28$ & $1.501(7)$ & $\mathrm{C} 38 \mathrm{~A}-\mathrm{C} 39 \mathrm{~A}$ & 1.3896 \\
\hline $\mathrm{C} 28-\mathrm{H} 28 \mathrm{~A}$ & 0.9900 & C39A-H39A & 0.9500 \\
\hline $\mathrm{C} 28-\mathrm{H} 28 \mathrm{~B}$ & 0.9900 & $\mathrm{C} 39 \mathrm{~A}-\mathrm{C} 40 \mathrm{~A}$ & 1.3910 \\
\hline
\end{tabular}




\begin{tabular}{|c|c|c|c|}
\hline $\mathrm{C} 43 \mathrm{~A}-\mathrm{H} 43 \mathrm{~A}$ & 0.9800 & $\mathrm{C} 40 \mathrm{~A}-\mathrm{H} 40 \mathrm{~A}$ & 0.9500 \\
\hline $\mathrm{C} 43 \mathrm{~A}-\mathrm{H} 43 \mathrm{~B}$ & 0.9800 & $\mathrm{C} 40 \mathrm{~A}-\mathrm{C} 41 \mathrm{~A}$ & 1.3900 \\
\hline $\mathrm{C} 43 \mathrm{~A}-\mathrm{H} 43 \mathrm{C}$ & 0.9800 & $\mathrm{C} 41 \mathrm{~A}-\mathrm{H} 41 \mathrm{~A}$ & 0.9500 \\
\hline $\mathrm{C} 43 \mathrm{~A}-\mathrm{C} 44 \mathrm{~A}$ & 1.4193 & $\mathrm{C} 41 \mathrm{~A}-\mathrm{C} 42 \mathrm{~A}$ & 1.3904 \\
\hline $\mathrm{C} 44 \mathrm{~A}-\mathrm{C} 45 \mathrm{~A}$ & 1.3920 & $\mathrm{C} 42 \mathrm{~A}-\mathrm{H} 42 \mathrm{~A}$ & 0.9500 \\
\hline $\mathrm{C} 44 \mathrm{~A}-\mathrm{C} 49 \mathrm{~A}$ & 1.3888 & & \\
\hline $\mathrm{O} 1-\mathrm{Yb} 1-\mathrm{O} 2$ & $82.10(11)$ & $\mathrm{C} 45 \mathrm{~A}-\mathrm{C} 44 \mathrm{~A}-\mathrm{C} 43 \mathrm{~A}$ & 119.3 \\
\hline $\mathrm{O} 1-\mathrm{Yb} 1-\mathrm{O} 5$ & $86.34(12)$ & $\mathrm{C} 49 \mathrm{~A}-\mathrm{C} 44 \mathrm{~A}-\mathrm{C} 43 \mathrm{~A}$ & 120.5 \\
\hline $\mathrm{O} 1-\mathrm{Yb} 1-\mathrm{O} 7$ & $77.59(12)$ & $\mathrm{C} 49 \mathrm{~A}-\mathrm{C} 44 \mathrm{~A}-\mathrm{C} 45 \mathrm{~A}$ & 120.0 \\
\hline $\mathrm{O} 1-\mathrm{Yb} 1-\mathrm{O} 6 \mathrm{~A}$ & $158.2(3)$ & $\mathrm{C} 44 \mathrm{~A}-\mathrm{C} 45 \mathrm{~A}-\mathrm{H} 45 \mathrm{~A}$ & 120.0 \\
\hline $\mathrm{O} 1-\mathrm{Yb} 1-\mathrm{O} 6 \mathrm{~B}$ & $165.6(4)$ & $\mathrm{C} 46 \mathrm{~A}-\mathrm{C} 45 \mathrm{~A}-\mathrm{C} 44 \mathrm{~A}$ & 119.9 \\
\hline $\mathrm{O} 2-\mathrm{Yb} 1-\mathrm{O} 5$ & $168.04(11)$ & $\mathrm{C} 46 \mathrm{~A}-\mathrm{C} 45 \mathrm{~A}-\mathrm{H} 45 \mathrm{~A}$ & 120.0 \\
\hline $\mathrm{O} 2-\mathrm{Yb} 1-\mathrm{O} 6 \mathrm{~A}$ & $100.6(5)$ & $\mathrm{C} 45 \mathrm{~A}-\mathrm{C} 46 \mathrm{~A}-\mathrm{H} 46 \mathrm{~A}$ & 120.0 \\
\hline $\mathrm{O} 2-\mathrm{Yb} 1-\mathrm{O} 6 \mathrm{~B}$ & $100.4(6)$ & $\mathrm{C} 45 \mathrm{~A}-\mathrm{C} 46 \mathrm{~A}-\mathrm{C} 47 \mathrm{~A}$ & 120.1 \\
\hline $\mathrm{O} 3-\mathrm{Yb} 1-\mathrm{O} 1$ & $106.85(12)$ & $\mathrm{C} 47 \mathrm{~A}-\mathrm{C} 46 \mathrm{~A}-\mathrm{H} 46 \mathrm{~A}$ & 120.0 \\
\hline $\mathrm{O} 3-\mathrm{Yb} 1-\mathrm{O} 2$ & $94.92(12)$ & $\mathrm{C} 46 \mathrm{~A}-\mathrm{C} 47 \mathrm{~A}-\mathrm{H} 47 \mathrm{~A}$ & 120.0 \\
\hline $\mathrm{O} 3-\mathrm{Yb} 1-\mathrm{O} 5$ & $85.50(12)$ & $\mathrm{C} 48 \mathrm{~A}-\mathrm{C} 47 \mathrm{~A}-\mathrm{C} 46 \mathrm{~A}$ & 120.0 \\
\hline $\mathrm{O} 3-\mathrm{Yb} 1-\mathrm{O} 7$ & $170.39(12)$ & $\mathrm{C} 48 \mathrm{~A}-\mathrm{C} 47 \mathrm{~A}-\mathrm{H} 47 \mathrm{~A}$ & 120.0 \\
\hline $\mathrm{O} 3-\mathrm{Yb} 1-\mathrm{O} 6 \mathrm{~A}$ & $94.5(3)$ & $\mathrm{C} 47 \mathrm{~A}-\mathrm{C} 48 \mathrm{~A}-\mathrm{H} 48 \mathrm{~A}$ & 120.0 \\
\hline $\mathrm{O} 3-\mathrm{Yb} 1-\mathrm{O} 6 \mathrm{~B}$ & $87.2(4)$ & $\mathrm{C} 47 \mathrm{~A}-\mathrm{C} 48 \mathrm{~A}-\mathrm{C} 49 \mathrm{~A}$ & 120.0 \\
\hline $\mathrm{O} 5-\mathrm{Yb} 1-\mathrm{O} 6 \mathrm{~A}$ & $91.2(5)$ & $\mathrm{C} 49 \mathrm{~A}-\mathrm{C} 48 \mathrm{~A}-\mathrm{H} 48 \mathrm{~A}$ & 120.0 \\
\hline $\mathrm{O} 5-\mathrm{Yb} 1-\mathrm{O} 6 \mathrm{~B}$ & $91.6(6)$ & $\mathrm{C} 44 \mathrm{~A}-\mathrm{C} 49 \mathrm{~A}-\mathrm{C} 48 \mathrm{~A}$ & 120.0 \\
\hline $\mathrm{O} 7-\mathrm{Yb} 1-\mathrm{O} 2$ & $77.07(11)$ & $\mathrm{C} 44 \mathrm{~A}-\mathrm{C} 49 \mathrm{~A}-\mathrm{H} 49 \mathrm{~A}$ & 120.0 \\
\hline $\mathrm{O} 7-\mathrm{Yb} 1-\mathrm{O} 5$ & $103.45(11)$ & $\mathrm{C} 48 \mathrm{~A}-\mathrm{C} 49 \mathrm{~A}-\mathrm{H} 49 \mathrm{~A}$ & 120.0 \\
\hline $\mathrm{O} 7-\mathrm{Yb} 1-\mathrm{O} 6 \mathrm{~A}$ & $82.0(3)$ & $\mathrm{C} 21 \mathrm{~A}-\mathrm{O} 6 \mathrm{~A}-\mathrm{Yb} 1$ & $130.8(10)$ \\
\hline $\mathrm{O} 7-\mathrm{Yb} 1-\mathrm{O} 6 \mathrm{~B}$ & $89.1(4)$ & $\mathrm{C} 21 \mathrm{~A}-\mathrm{O} 6 \mathrm{~A}-\mathrm{C} 24 \mathrm{~A}$ & $105.8(10)$ \\
\hline $\mathrm{O} 1-\mathrm{Yb} 2-\mathrm{O} 7$ & $69.99(10)$ & $\mathrm{C} 24 \mathrm{~A}-\mathrm{O} 6 \mathrm{~A}-\mathrm{Yb} 1$ & $120.3(9)$ \\
\hline $\mathrm{O} 2-\mathrm{Yb} 2-\mathrm{O} 1$ & $75.17(10)$ & $\mathrm{O} 7-\mathrm{C} 1 \mathrm{E}-\mathrm{C} 2 \mathrm{E}$ & $112.7(4)$ \\
\hline $\mathrm{O} 2-\mathrm{Yb} 2-\mathrm{O} 7$ & $71.36(11)$ & $\mathrm{O} 7-\mathrm{C} 1 \mathrm{E}-\mathrm{C} 6 \mathrm{E}$ & $127.3(4)$ \\
\hline $\mathrm{O} 4-\mathrm{Yb} 2-\mathrm{O} 1$ & $137.75(12)$ & $\mathrm{C} 2 \mathrm{E}-\mathrm{C} 1 \mathrm{E}-\mathrm{C} 6 \mathrm{E}$ & 120.0 \\
\hline $\mathrm{O} 4-\mathrm{Yb} 2-\mathrm{O} 2$ & $147.05(12)$ & $\mathrm{C} 1 \mathrm{E}-\mathrm{C} 2 \mathrm{E}-\mathrm{H} 2 \mathrm{E}$ & 120.0 \\
\hline $\mathrm{O} 4-\mathrm{Yb} 2-\mathrm{O} 7$ & $115.61(11)$ & $\mathrm{C} 3 \mathrm{E}-\mathrm{C} 2 \mathrm{E}-\mathrm{C} 1 \mathrm{E}$ & 120.0 \\
\hline $\mathrm{Yb} 1-\mathrm{O} 1-\mathrm{Yb} 2$ & $89.98(11)$ & $\mathrm{C} 3 \mathrm{E}-\mathrm{C} 2 \mathrm{E}-\mathrm{H} 2 \mathrm{E}$ & 120.0 \\
\hline $\mathrm{Yb} 1-\mathrm{O} 2-\mathrm{Yb} 2$ & $90.47(11)$ & $\mathrm{C} 2 \mathrm{E}-\mathrm{C} 3 \mathrm{E}-\mathrm{H} 3 \mathrm{E}$ & 120.0 \\
\hline $\mathrm{Yb} 1-\mathrm{O} 7-\mathrm{Yb} 2$ & $88.99(11)$ & $\mathrm{C} 2 \mathrm{E}-\mathrm{C} 3 \mathrm{E}-\mathrm{C} 4 \mathrm{E}$ & 120.0 \\
\hline $\mathrm{C} 1 \mathrm{D}-\mathrm{O} 1-\mathrm{Yb} 1$ & $139.0(3)$ & $\mathrm{C} 4 \mathrm{E}-\mathrm{C} 3 \mathrm{E}-\mathrm{H} 3 \mathrm{E}$ & 120.0 \\
\hline $\mathrm{C} 1 \mathrm{D}-\mathrm{O} 1-\mathrm{Yb} 2$ & $115.6(3)$ & $\mathrm{C} 3 \mathrm{E}-\mathrm{C} 4 \mathrm{E}-\mathrm{H} 4 \mathrm{E}$ & 120.0 \\
\hline $\mathrm{C} 1 \mathrm{~B}-\mathrm{O} 2-\mathrm{Yb} 1$ & $125.2(3)$ & $\mathrm{C} 5 \mathrm{E}-\mathrm{C} 4 \mathrm{E}-\mathrm{C} 3 \mathrm{E}$ & 120.0 \\
\hline $\mathrm{C} 1 \mathrm{~B}-\mathrm{O} 2-\mathrm{Yb} 2$ & $126.8(3)$ & $\mathrm{C} 5 \mathrm{E}-\mathrm{C} 4 \mathrm{E}-\mathrm{H} 4 \mathrm{E}$ & 120.0 \\
\hline $\mathrm{C} 1 \mathrm{~A}-\mathrm{O} 3-\mathrm{Yb} 1$ & $162.0(3)$ & $\mathrm{C} 4 \mathrm{E}-\mathrm{C} 5 \mathrm{E}-\mathrm{H} 5 \mathrm{E}$ & 120.0 \\
\hline $\mathrm{C} 1 \mathrm{C}-\mathrm{O} 4-\mathrm{Yb} 2$ & $166.3(3)$ & $\mathrm{C} 4 \mathrm{E}-\mathrm{C} 5 \mathrm{E}-\mathrm{C} 6 \mathrm{E}$ & 120.0 \\
\hline $\mathrm{C} 25-\mathrm{O} 5-\mathrm{Yb} 1$ & $121.4(3)$ & $\mathrm{C} 6 \mathrm{E}-\mathrm{C} 5 \mathrm{E}-\mathrm{H} 5 \mathrm{E}$ & 120.0 \\
\hline $\mathrm{C} 28-\mathrm{O} 5-\mathrm{Yb} 1$ & $132.8(3)$ & $\mathrm{C} 1 \mathrm{E}-\mathrm{C} 6 \mathrm{E}-\mathrm{C} 7 \mathrm{E}$ & $119.1(4)$ \\
\hline $\mathrm{C} 28-\mathrm{O} 5-\mathrm{C} 25$ & $105.8(4)$ & $\mathrm{C} 5 \mathrm{E}-\mathrm{C} 6 \mathrm{E}-\mathrm{C} 1 \mathrm{E}$ & 120.0 \\
\hline $\mathrm{C} 1 \mathrm{E}-\mathrm{O} 7-\mathrm{Yb} 1$ & $144.7(4)$ & $\mathrm{C} 5 \mathrm{E}-\mathrm{C} 6 \mathrm{E}-\mathrm{C} 7 \mathrm{E}$ & $120.9(4)$ \\
\hline $\mathrm{C} 1 \mathrm{E}-\mathrm{O} 7-\mathrm{Yb} 2$ & $113.1(3)$ & $\mathrm{C} 8 \mathrm{E}-\mathrm{C} 7 \mathrm{E}-\mathrm{C} 6 \mathrm{E}$ & $119.6(3)$ \\
\hline $\mathrm{C} 1 \mathrm{~F}-\mathrm{O} 7-\mathrm{Yb} 1$ & $125.5(4)$ & $\mathrm{C} 20 \mathrm{E}-\mathrm{C} 7 \mathrm{E}-\mathrm{C} 6 \mathrm{E}$ & $120.1(3)$ \\
\hline
\end{tabular}




\begin{tabular}{|c|c|}
\hline $\mathrm{C} 1 \mathrm{~F}-\mathrm{O} 7-\mathrm{Yb} 2$ & $116.5(4)$ \\
\hline $\mathrm{O} 3-\mathrm{C} 1 \mathrm{~A}-\mathrm{C} 2 \mathrm{~A}$ & $121.2(4)$ \\
\hline $\mathrm{O} 3-\mathrm{C} 1 \mathrm{~A}-\mathrm{C} 6 \mathrm{~A}$ & $120.3(4)$ \\
\hline $\mathrm{C} 2 \mathrm{~A}-\mathrm{C} 1 \mathrm{~A}-\mathrm{C} 6 \mathrm{~A}$ & $118.5(4)$ \\
\hline $\mathrm{O} 2-\mathrm{C} 1 \mathrm{~B}-\mathrm{C} 2 \mathrm{~B}$ & $120.0(4)$ \\
\hline $\mathrm{O} 2-\mathrm{C} 1 \mathrm{~B}-\mathrm{C} 6 \mathrm{~B}$ & $120.7(4)$ \\
\hline $\mathrm{C} 2 \mathrm{~B}-\mathrm{C} 1 \mathrm{~B}-\mathrm{C} 6 \mathrm{~B}$ & $119.2(4)$ \\
\hline $\mathrm{O} 4-\mathrm{C} 1 \mathrm{C}-\mathrm{C} 2 \mathrm{C}$ & $120.3(5)$ \\
\hline $\mathrm{O} 4-\mathrm{C} 1 \mathrm{C}-\mathrm{C} 6 \mathrm{C}$ & $121.9(4)$ \\
\hline $\mathrm{C} 2 \mathrm{C}-\mathrm{C} 1 \mathrm{C}-\mathrm{C} 6 \mathrm{C}$ & $117.8(4)$ \\
\hline $\mathrm{O} 1-\mathrm{C} 1 \mathrm{D}-\mathrm{C} 2 \mathrm{D}$ & $120.6(4)$ \\
\hline $\mathrm{O} 1-\mathrm{C} 1 \mathrm{D}-\mathrm{C} 6 \mathrm{D}$ & $120.5(4)$ \\
\hline $\mathrm{C} 2 \mathrm{D}-\mathrm{C} 1 \mathrm{D}-\mathrm{C} 6 \mathrm{D}$ & $118.8(4)$ \\
\hline $\mathrm{C} 1 \mathrm{~A}-\mathrm{C} 2 \mathrm{~A}-\mathrm{H} 2 \mathrm{~A}$ & 119.5 \\
\hline $\mathrm{C} 3 \mathrm{~A}-\mathrm{C} 2 \mathrm{~A}-\mathrm{C} 1 \mathrm{~A}$ & $121.0(4)$ \\
\hline $\mathrm{C} 3 \mathrm{~A}-\mathrm{C} 2 \mathrm{~A}-\mathrm{H} 2 \mathrm{~A}$ & 119.5 \\
\hline $\mathrm{C} 1 \mathrm{~B}-\mathrm{C} 2 \mathrm{~B}-\mathrm{H} 2 \mathrm{~B}$ & 119.4 \\
\hline $\mathrm{C} 3 \mathrm{~B}-\mathrm{C} 2 \mathrm{~B}-\mathrm{C} 1 \mathrm{~B}$ & $121.1(4)$ \\
\hline $\mathrm{C} 3 \mathrm{~B}-\mathrm{C} 2 \mathrm{~B}-\mathrm{H} 2 \mathrm{~B}$ & 119.4 \\
\hline $\mathrm{C} 1 \mathrm{C}-\mathrm{C} 2 \mathrm{C}-\mathrm{H} 2 \mathrm{C}$ & 119.4 \\
\hline $\mathrm{C} 3 \mathrm{C}-\mathrm{C} 2 \mathrm{C}-\mathrm{C} 1 \mathrm{C}$ & $121.1(5)$ \\
\hline $\mathrm{C} 3 \mathrm{C}-\mathrm{C} 2 \mathrm{C}-\mathrm{H} 2 \mathrm{C}$ & 119.4 \\
\hline $\mathrm{C} 1 \mathrm{D}-\mathrm{C} 2 \mathrm{D}-\mathrm{H} 2 \mathrm{D}$ & 119.6 \\
\hline $\mathrm{C} 3 \mathrm{D}-\mathrm{C} 2 \mathrm{D}-\mathrm{C} 1 \mathrm{D}$ & $120.9(5)$ \\
\hline $\mathrm{C} 3 \mathrm{D}-\mathrm{C} 2 \mathrm{D}-\mathrm{H} 2 \mathrm{D}$ & 119.6 \\
\hline $\mathrm{C} 2 \mathrm{~A}-\mathrm{C} 3 \mathrm{~A}-\mathrm{H} 3 \mathrm{~A}$ & 119.4 \\
\hline $\mathrm{C} 4 \mathrm{~A}-\mathrm{C} 3 \mathrm{~A}-\mathrm{C} 2 \mathrm{~A}$ & $121.3(5)$ \\
\hline $\mathrm{C} 4 \mathrm{~A}-\mathrm{C} 3 \mathrm{~A}-\mathrm{H} 3 \mathrm{~A}$ & 119.4 \\
\hline $\mathrm{C} 2 \mathrm{~B}-\mathrm{C} 3 \mathrm{~B}-\mathrm{H} 3 \mathrm{~B}$ & 120.0 \\
\hline $\mathrm{C} 2 \mathrm{~B}-\mathrm{C} 3 \mathrm{~B}-\mathrm{C} 4 \mathrm{~B}$ & $120.0(4)$ \\
\hline $\mathrm{C} 4 \mathrm{~B}-\mathrm{C} 3 \mathrm{~B}-\mathrm{H} 3 \mathrm{~B}$ & 120.0 \\
\hline $\mathrm{C} 2 \mathrm{C}-\mathrm{C} 3 \mathrm{C}-\mathrm{H} 3 \mathrm{C}$ & 119.5 \\
\hline $\mathrm{C} 4 \mathrm{C}-\mathrm{C} 3 \mathrm{C}-\mathrm{C} 2 \mathrm{C}$ & $120.9(5)$ \\
\hline $\mathrm{C} 4 \mathrm{C}-\mathrm{C} 3 \mathrm{C}-\mathrm{H} 3 \mathrm{C}$ & 119.5 \\
\hline $\mathrm{C} 2 \mathrm{D}-\mathrm{C} 3 \mathrm{D}-\mathrm{H} 3 \mathrm{D}$ & 119.8 \\
\hline $\mathrm{C} 4 \mathrm{D}-\mathrm{C} 3 \mathrm{D}-\mathrm{C} 2 \mathrm{D}$ & $120.4(5)$ \\
\hline $\mathrm{C} 4 \mathrm{D}-\mathrm{C} 3 \mathrm{D}-\mathrm{H} 3 \mathrm{D}$ & 119.8 \\
\hline $\mathrm{C} 3 \mathrm{~A}-\mathrm{C} 4 \mathrm{~A}-\mathrm{H} 4 \mathrm{~A}$ & 120.8 \\
\hline $\mathrm{C} 3 \mathrm{~A}-\mathrm{C} 4 \mathrm{~A}-\mathrm{C} 5 \mathrm{~A}$ & $118.5(4)$ \\
\hline $\mathrm{C} 5 \mathrm{~A}-\mathrm{C} 4 \mathrm{~A}-\mathrm{H} 4 \mathrm{~A}$ & 120.8 \\
\hline $\mathrm{C} 3 \mathrm{~B}-\mathrm{C} 4 \mathrm{~B}-\mathrm{H} 4 \mathrm{~B}$ & 120.5 \\
\hline $\mathrm{C} 3 \mathrm{~B}-\mathrm{C} 4 \mathrm{~B}-\mathrm{C} 5 \mathrm{~B}$ & $119.0(5)$ \\
\hline $\mathrm{C} 5 \mathrm{~B}-\mathrm{C} 4 \mathrm{~B}-\mathrm{H} 4 \mathrm{~B}$ & 120.5 \\
\hline $\mathrm{C} 3 \mathrm{C}-\mathrm{C} 4 \mathrm{C}-\mathrm{H} 4 \mathrm{C}$ & 120.7 \\
\hline $\mathrm{C} 3 \mathrm{C}-\mathrm{C} 4 \mathrm{C}-\mathrm{C} 5 \mathrm{C}$ & $118.5(5)$ \\
\hline $\mathrm{C} 5 \mathrm{C}-\mathrm{C} 4 \mathrm{C}-\mathrm{H} 4 \mathrm{C}$ & 120.7 \\
\hline $\mathrm{C} 3 \mathrm{D}-\mathrm{C} 4 \mathrm{D}-\mathrm{H} 4 \mathrm{D}$ & 120.2 \\
\hline $\mathrm{C} 3 \mathrm{D}-\mathrm{C} 4 \mathrm{D}-\mathrm{C} 5 \mathrm{D}$ & $119.6(5)$ \\
\hline
\end{tabular}

$\begin{array}{ll}\text { C20E-C7E-C8E } & 119.1 \\ \text { C15E-C16E-H16E } & 118.8 \\ \text { C17E-C16E-H16E } & 118.8 \\ \text { C17E-C16E-C15E } & 122.3 \\ \text { C7E-C8E-C9E } & 120.5 \\ \text { C13E-C8E-C7E } & 120.0 \\ \text { C13E-C8E-C9E } & 119.5 \\ \text { C8E-C9E-H9E } & 120.0 \\ \text { C10E-C9E-C8E } & 120.0 \\ \text { C10E-C9E-H9E } & 120.0 \\ \text { C9E-C10E-H10E } & 119.9 \\ \text { C9E-C10E-C11E } & 120.3 \\ \text { C11E-C10E-H10E } & 119.9 \\ \text { C10E-C11E-H11E } & 119.5 \\ \text { C12E-C11E-C10E } & 121.0 \\ \text { C12E-C11E-H11E } & 119.5 \\ \text { C11E-C12E-H12E } & 120.0 \\ \text { C11E-C12E-C13E } & 120.0 \\ \text { C13E-C12E-H12E } & 120.0 \\ \text { C8E-C13E-C12E } & 119.0 \\ \text { C14E-C13E-C8E } & 119.5 \\ \text { C14E-C13E-C12E } & 121.5 \\ \text { C13E-C14E-H14E } & 119.5 \\ \text { C15E-C14E-C13E } & 121.0 \\ \text { C15E-C14E-H14E } & 119.5 \\ \text { C14E-C15E-C16E } & 122.5 \\ \text { C14E-C15E-C20E } & 120.6 \\ \text { C20E-C15E-C16E } & 116.9 \\ \text { C16E-C17E-H17E } & 119.7 \\ \text { C16E-C17E-C18E } & 120.7 \\ \text { C18E-C17E-H17E } & 119.7 \\ \text { C17E-C18E-H18E } & 119.9 \\ \text { C19E-C18E-C17E } & 120.1 \\ \text { C19E-C18E-H18E } & 119.9 \\ \text { C18E-C19E-H19E } & 119.7 \\ \text { C18E-C19E-C20E } & 120.5 \\ \text { C20E-C19E-H19E } & 119.7 \\ \text { C7E-C20E-C15E } & 119.6 \\ \text { C7E-C20E-C19E } & 121.2 \\ \text { C15E-C20E-C19E } & 119.3 \\ \text { O6A-C21A-H21A } & 110.7 \\ \text { O6A-C21A-H21B } & 110.7 \\ \text { O6A-C21A-C22A } & 105.4 \\ \text { H21A-C21A-H21B } & 108.8 \\ \text { C22A-C21A-H21A } & 110.7 \\ \text { C22A-C21A-H21B } & 110.7 \\ \text { C21A-C22A-H22A } & \\ \text { C21A-C22A-H22B } & \\ & \\ & \end{array}$




\begin{tabular}{|c|c|}
\hline $\mathrm{C} 5 \mathrm{D}-\mathrm{C} 4 \mathrm{D}-\mathrm{H} 4 \mathrm{D}$ & 120.2 \\
\hline $\mathrm{C} 4 \mathrm{~A}-\mathrm{C} 5 \mathrm{~A}-\mathrm{H} 5 \mathrm{~A}$ & 118.8 \\
\hline $\mathrm{C} 4 \mathrm{~A}-\mathrm{C} 5 \mathrm{~A}-\mathrm{C} 6 \mathrm{~A}$ & $122.3(4)$ \\
\hline $\mathrm{C} 6 \mathrm{~A}-\mathrm{C} 5 \mathrm{~A}-\mathrm{H} 5 \mathrm{~A}$ & 118.8 \\
\hline $\mathrm{C} 4 \mathrm{~B}-\mathrm{C} 5 \mathrm{~B}-\mathrm{H} 5 \mathrm{~B}$ & 119.0 \\
\hline $\mathrm{C} 4 \mathrm{~B}-\mathrm{C} 5 \mathrm{~B}-\mathrm{C} 6 \mathrm{~B}$ & $122.0(5)$ \\
\hline $\mathrm{C} 6 \mathrm{~B}-\mathrm{C} 5 \mathrm{~B}-\mathrm{H} 5 \mathrm{~B}$ & 119.0 \\
\hline $\mathrm{C} 4 \mathrm{C}-\mathrm{C} 5 \mathrm{C}-\mathrm{H} 5 \mathrm{C}$ & 118.9 \\
\hline $\mathrm{C} 6 \mathrm{C}-\mathrm{C} 5 \mathrm{C}-\mathrm{C} 4 \mathrm{C}$ & $122.2(5)$ \\
\hline $\mathrm{C} 6 \mathrm{C}-\mathrm{C} 5 \mathrm{C}-\mathrm{H} 5 \mathrm{C}$ & 118.9 \\
\hline $\mathrm{C} 4 \mathrm{D}-\mathrm{C} 5 \mathrm{D}-\mathrm{H} 5 \mathrm{D}$ & 119.6 \\
\hline $\mathrm{C} 4 \mathrm{D}-\mathrm{C} 5 \mathrm{D}-\mathrm{C} 6 \mathrm{D}$ & $120.9(5)$ \\
\hline $\mathrm{C} 6 \mathrm{D}-\mathrm{C} 5 \mathrm{D}-\mathrm{H} 5 \mathrm{D}$ & 119.6 \\
\hline $\mathrm{C} 1 \mathrm{~A}-\mathrm{C} 6 \mathrm{~A}-\mathrm{C} 7 \mathrm{~A}$ & $123.2(4)$ \\
\hline $\mathrm{C} 5 \mathrm{~A}-\mathrm{C} 6 \mathrm{~A}-\mathrm{C} 1 \mathrm{~A}$ & $118.3(4)$ \\
\hline $\mathrm{C} 5 \mathrm{~A}-\mathrm{C} 6 \mathrm{~A}-\mathrm{C} 7 \mathrm{~A}$ & $118.4(4)$ \\
\hline $\mathrm{C} 1 \mathrm{~B}-\mathrm{C} 6 \mathrm{~B}-\mathrm{C} 7 \mathrm{~B}$ & $120.5(4)$ \\
\hline $\mathrm{C} 5 \mathrm{~B}-\mathrm{C} 6 \mathrm{~B}-\mathrm{C} 1 \mathrm{~B}$ & $118.3(4)$ \\
\hline $\mathrm{C} 5 \mathrm{~B}-\mathrm{C} 6 \mathrm{~B}-\mathrm{C} 7 \mathrm{~B}$ & $121.0(4)$ \\
\hline $\mathrm{C} 1 \mathrm{C}-\mathrm{C} 6 \mathrm{C}-\mathrm{C} 7 \mathrm{C}$ & $120.9(4)$ \\
\hline $\mathrm{C} 5 \mathrm{C}-\mathrm{C} 6 \mathrm{C}-\mathrm{C} 1 \mathrm{C}$ & $119.4(5)$ \\
\hline $\mathrm{C} 5 \mathrm{C}-\mathrm{C} 6 \mathrm{C}-\mathrm{C} 7 \mathrm{C}$ & $119.7(4)$ \\
\hline $\mathrm{C} 1 \mathrm{D}-\mathrm{C} 6 \mathrm{D}-\mathrm{C} 7 \mathrm{D}$ & $119.8(4)$ \\
\hline $\mathrm{C} 5 \mathrm{D}-\mathrm{C} 6 \mathrm{D}-\mathrm{C} 1 \mathrm{D}$ & $119.4(5)$ \\
\hline $\mathrm{C} 5 \mathrm{D}-\mathrm{C} 6 \mathrm{D}-\mathrm{C} 7 \mathrm{D}$ & $120.8(5)$ \\
\hline $\mathrm{C} 8 \mathrm{~A}-\mathrm{C} 7 \mathrm{~A}-\mathrm{C} 6 \mathrm{~A}$ & $120.2(4)$ \\
\hline $\mathrm{C} 8 \mathrm{~A}-\mathrm{C} 7 \mathrm{~A}-\mathrm{C} 20 \mathrm{~A}$ & $119.4(4)$ \\
\hline $\mathrm{C} 20 \mathrm{~A}-\mathrm{C} 7 \mathrm{~A}-\mathrm{C} 6 \mathrm{~A}$ & $120.2(4)$ \\
\hline $\mathrm{C} 8 \mathrm{~B}-\mathrm{C} 7 \mathrm{~B}-\mathrm{C} 6 \mathrm{~B}$ & $121.7(4)$ \\
\hline $\mathrm{C} 8 \mathrm{~B}-\mathrm{C} 7 \mathrm{~B}-\mathrm{C} 20 \mathrm{~B}$ & $119.9(4)$ \\
\hline $\mathrm{C} 20 \mathrm{~B}-\mathrm{C} 7 \mathrm{~B}-\mathrm{C} 6 \mathrm{~B}$ & $118.3(4)$ \\
\hline $\mathrm{C} 8 \mathrm{C}-\mathrm{C} 7 \mathrm{C}-\mathrm{C} 6 \mathrm{C}$ & $120.7(4)$ \\
\hline $\mathrm{C} 20 \mathrm{C}-\mathrm{C} 7 \mathrm{C}-\mathrm{C} 6 \mathrm{C}$ & $120.7(4)$ \\
\hline $\mathrm{C} 20 \mathrm{C}-\mathrm{C} 7 \mathrm{C}-\mathrm{C} 8 \mathrm{C}$ & $118.6(5)$ \\
\hline $\mathrm{C} 8 \mathrm{D}-\mathrm{C} 7 \mathrm{D}-\mathrm{C} 6 \mathrm{D}$ & $120.5(5)$ \\
\hline $\mathrm{C} 20 \mathrm{D}-\mathrm{C} 7 \mathrm{D}-\mathrm{C} 6 \mathrm{D}$ & $119.6(4)$ \\
\hline$C 20 D-C 7 D-C 8 D$ & $119.8(5)$ \\
\hline $\mathrm{C} 7 \mathrm{~A}-\mathrm{C} 8 \mathrm{~A}-\mathrm{C} 9 \mathrm{~A}$ & $121.7(4)$ \\
\hline $\mathrm{C} 7 \mathrm{~A}-\mathrm{C} 8 \mathrm{~A}-\mathrm{C} 13 \mathrm{~A}$ & $120.0(4)$ \\
\hline $\mathrm{C} 9 \mathrm{~A}-\mathrm{C} 8 \mathrm{~A}-\mathrm{C} 13 \mathrm{~A}$ & $118.3(4)$ \\
\hline $\mathrm{C} 7 \mathrm{~B}-\mathrm{C} 8 \mathrm{~B}-\mathrm{C} 9 \mathrm{~B}$ & $123.2(4)$ \\
\hline $\mathrm{C} 7 \mathrm{~B}-\mathrm{C} 8 \mathrm{~B}-\mathrm{C} 13 \mathrm{~B}$ & $118.9(4)$ \\
\hline $\mathrm{C} 9 \mathrm{~B}-\mathrm{C} 8 \mathrm{~B}-\mathrm{C} 13 \mathrm{~B}$ & $117.9(4)$ \\
\hline $\mathrm{C} 7 \mathrm{C}-\mathrm{C} 8 \mathrm{C}-\mathrm{C} 9 \mathrm{C}$ & $122.6(5)$ \\
\hline $\mathrm{C} 7 \mathrm{C}-\mathrm{C} 8 \mathrm{C}-\mathrm{C} 13 \mathrm{C}$ & $119.9(5)$ \\
\hline $\mathrm{C} 9 \mathrm{C}-\mathrm{C} 8 \mathrm{C}-\mathrm{C} 13 \mathrm{C}$ & $117.5(5)$ \\
\hline $\mathrm{C} 7 \mathrm{D}-\mathrm{C} 8 \mathrm{D}-\mathrm{C} 9 \mathrm{D}$ & $122.6(6)$ \\
\hline $\mathrm{C} 13 \mathrm{D}-\mathrm{C} 8 \mathrm{D}-\mathrm{C} 7 \mathrm{D}$ & $119.6(6)$ \\
\hline
\end{tabular}

$\begin{array}{ll}\mathrm{H} 22 \mathrm{~A}-\mathrm{C} 22 \mathrm{~A}-\mathrm{H} 22 \mathrm{~B} & 108.7 \\ \mathrm{C} 23 \mathrm{~A}-\mathrm{C} 22 \mathrm{~A}-\mathrm{C} 21 \mathrm{~A} & 105.7(14) \\ \mathrm{C} 23 \mathrm{~A}-\mathrm{C} 22 \mathrm{~A}-\mathrm{H} 22 \mathrm{~A} & 110.6 \\ \mathrm{C} 23 \mathrm{~A}-\mathrm{C} 22 \mathrm{~A}-\mathrm{H} 22 \mathrm{~B} & 110.6 \\ \mathrm{C} 22 \mathrm{~A}-\mathrm{C} 23 \mathrm{~A}-\mathrm{H} 23 \mathrm{~A} & 110.5 \\ \mathrm{C} 22 \mathrm{~A}-\mathrm{C} 23 \mathrm{~A}-\mathrm{H} 23 \mathrm{~B} & 110.5 \\ \mathrm{H} 23 \mathrm{~A}-\mathrm{C} 23 \mathrm{~A}-\mathrm{H} 23 \mathrm{~B} & 108.6 \\ \mathrm{C} 24 \mathrm{~A}-\mathrm{C} 23 \mathrm{~A}-\mathrm{C} 22 \mathrm{~A} & 106.4(13) \\ \mathrm{C} 24 \mathrm{~A}-\mathrm{C} 23 \mathrm{~A}-\mathrm{H} 23 \mathrm{~A} & 110.5 \\ \mathrm{C} 24 \mathrm{~A}-\mathrm{C} 23 \mathrm{~A}-\mathrm{H} 23 \mathrm{~B} & 110.5 \\ \mathrm{O} 6 \mathrm{~A}-\mathrm{C} 24 \mathrm{~A}-\mathrm{H} 24 \mathrm{~A} & 109.2 \\ \mathrm{O} 6 \mathrm{~A}-\mathrm{C} 24 \mathrm{~A}-\mathrm{H} 24 \mathrm{~B} & 109.2 \\ \mathrm{C} 23 \mathrm{~A}-\mathrm{C} 24 \mathrm{~A}-\mathrm{O} 6 \mathrm{~A} & 112.0(10)\end{array}$

$\mathrm{C} 23 \mathrm{~A}-\mathrm{C} 24 \mathrm{~A}-\mathrm{H} 24 \mathrm{~A} \quad 109.2$

$\mathrm{C} 23 \mathrm{~A}-\mathrm{C} 24 \mathrm{~A}-\mathrm{H} 24 \mathrm{~B} \quad 109.2$

$\mathrm{H} 24 \mathrm{~A}-\mathrm{C} 24 \mathrm{~A}-\mathrm{H} 24 \mathrm{~B} \quad 107.9$

$\mathrm{H} 29 \mathrm{~A}-\mathrm{C} 29 \mathrm{~A}-\mathrm{H} 29 \mathrm{~B} \quad 109.5$

$\mathrm{H} 29 \mathrm{~A}-\mathrm{C} 29 \mathrm{~A}-\mathrm{H} 29 \mathrm{C} \quad 109.5$

$\mathrm{H} 29 \mathrm{~B}-\mathrm{C} 29 \mathrm{~A}-\mathrm{H} 29 \mathrm{C}$

$\mathrm{C} 30 \mathrm{~A}-\mathrm{C} 29 \mathrm{~A}-\mathrm{H} 29 \mathrm{~A} \quad 109.5$

$\mathrm{C} 30 \mathrm{~A}-\mathrm{C} 29 \mathrm{~A}-\mathrm{H} 29 \mathrm{~B} \quad 109.5$

$\mathrm{C} 30 \mathrm{~A}-\mathrm{C} 29 \mathrm{~A}-\mathrm{H} 29 \mathrm{C} \quad 109.5$

$\mathrm{C} 31 \mathrm{~A}-\mathrm{C} 30 \mathrm{~A}-\mathrm{C} 29 \mathrm{~A} \quad 119.4$

$\mathrm{C} 35 \mathrm{~A}-\mathrm{C} 30 \mathrm{~A}-\mathrm{C} 29 \mathrm{~A} \quad 120.5$

$\mathrm{C} 35 \mathrm{~A}-\mathrm{C} 30 \mathrm{~A}-\mathrm{C} 31 \mathrm{~A} \quad 120.0$

$\mathrm{C} 30 \mathrm{~A}-\mathrm{C} 31 \mathrm{~A}-\mathrm{H} 31 \mathrm{~A} \quad 120.0$

$\mathrm{C} 30 \mathrm{~A}-\mathrm{C} 31 \mathrm{~A}-\mathrm{C} 32 \mathrm{~A} \quad 120.0$

$\mathrm{C} 32 \mathrm{~A}-\mathrm{C} 31 \mathrm{~A}-\mathrm{H} 31 \mathrm{~A} \quad 120.0$

$\mathrm{C} 31 \mathrm{~A}-\mathrm{C} 32 \mathrm{~A}-\mathrm{H} 32 \mathrm{~A} \quad 120.0$

$\mathrm{C} 33 \mathrm{~A}-\mathrm{C} 32 \mathrm{~A}-\mathrm{C} 31 \mathrm{~A} \quad 119.9$

$\mathrm{C} 33 \mathrm{~A}-\mathrm{C} 32 \mathrm{~A}-\mathrm{H} 32 \mathrm{~A} \quad 120.0$

$\mathrm{C} 32 \mathrm{~A}-\mathrm{C} 33 \mathrm{~A}-\mathrm{H} 33 \mathrm{~A} \quad 120.0$

$\mathrm{C} 34 \mathrm{~A}-\mathrm{C} 33 \mathrm{~A}-\mathrm{C} 32 \mathrm{~A} \quad 120.1$

$\mathrm{C} 34 \mathrm{~A}-\mathrm{C} 33 \mathrm{~A}-\mathrm{H} 33 \mathrm{~A} \quad 120.0$

$\mathrm{C} 33 \mathrm{~A}-\mathrm{C} 34 \mathrm{~A}-\mathrm{H} 34 \mathrm{~A} \quad 120.0$

$\mathrm{C} 33 \mathrm{~A}-\mathrm{C} 34 \mathrm{~A}-\mathrm{C} 35 \mathrm{~A} \quad 119.9$

$\mathrm{C} 35 \mathrm{~A}-\mathrm{C} 34 \mathrm{~A}-\mathrm{H} 34 \mathrm{~A} \quad 120.0$

$\mathrm{C} 30 \mathrm{~A}-\mathrm{C} 35 \mathrm{~A}-\mathrm{C} 34 \mathrm{~A} \quad 120.0$

$\mathrm{C} 30 \mathrm{~A}-\mathrm{C} 35 \mathrm{~A}-\mathrm{H} 35 \mathrm{~A} \quad 120.0$

$\mathrm{C} 34 \mathrm{~A}-\mathrm{C} 35 \mathrm{~A}-\mathrm{H} 35 \mathrm{~A} \quad 120.0$

$\mathrm{C} 21 \mathrm{~B}-\mathrm{O} 6 \mathrm{~B}-\mathrm{Yb} 1 \quad 129.0(14)$

$\mathrm{C} 21 \mathrm{~B}-\mathrm{O} 6 \mathrm{~B}-\mathrm{C} 24 \mathrm{~B} \quad 106.7(13)$

$\mathrm{C} 24 \mathrm{~B}-\mathrm{O} 6 \mathrm{~B}-\mathrm{Yb} 1 \quad 115.4(10)$

$\mathrm{C} 6 \mathrm{~F}-\mathrm{C} 5 \mathrm{~F}-\mathrm{H} 5 \mathrm{~F} \quad 120.0$

$\mathrm{C} 6 \mathrm{~F}-\mathrm{C} 5 \mathrm{~F}-\mathrm{C} 4 \mathrm{~F} \quad 120.0$

$\mathrm{C} 4 \mathrm{~F}-\mathrm{C} 5 \mathrm{~F}-\mathrm{H} 5 \mathrm{~F} \quad 120.0$

$\mathrm{C} 5 \mathrm{~F}-\mathrm{C} 6 \mathrm{~F}-\mathrm{C} 7 \mathrm{~F} \quad 118.0(5)$

$\mathrm{C} 1 \mathrm{~F}-\mathrm{C} 6 \mathrm{~F}-\mathrm{C} 5 \mathrm{~F} \quad 120.0$ 


\begin{tabular}{|c|c|}
\hline $\mathrm{C} 13 \mathrm{D}-\mathrm{C} 8 \mathrm{D}-\mathrm{C} 9 \mathrm{D}$ & $117.8(5)$ \\
\hline C8A-C9A-H9A & 119.3 \\
\hline $\mathrm{C} 10 \mathrm{~A}-\mathrm{C} 9 \mathrm{~A}-\mathrm{C} 8 \mathrm{~A}$ & $121.5(4)$ \\
\hline $\mathrm{C} 10 \mathrm{~A}-\mathrm{C} 9 \mathrm{~A}-\mathrm{H} 9 \mathrm{~A}$ & 119.3 \\
\hline $\mathrm{C} 8 \mathrm{~B}-\mathrm{C} 9 \mathrm{~B}-\mathrm{H} 9 \mathrm{~B}$ & 119.0 \\
\hline $\mathrm{C} 10 \mathrm{~B}-\mathrm{C} 9 \mathrm{~B}-\mathrm{C} 8 \mathrm{~B}$ & $121.9(5)$ \\
\hline $\mathrm{C} 10 \mathrm{~B}-\mathrm{C} 9 \mathrm{~B}-\mathrm{H} 9 \mathrm{~B}$ & 119.0 \\
\hline $\mathrm{C} 8 \mathrm{C}-\mathrm{C} 9 \mathrm{C}-\mathrm{H} 9 \mathrm{C}$ & 119.9 \\
\hline $\mathrm{C} 10 \mathrm{C}-\mathrm{C} 9 \mathrm{C}-\mathrm{C} 8 \mathrm{C}$ & $120.1(7)$ \\
\hline $\mathrm{C} 10 \mathrm{C}-\mathrm{C} 9 \mathrm{C}-\mathrm{H} 9 \mathrm{C}$ & 119.9 \\
\hline $\mathrm{C} 8 \mathrm{D}-\mathrm{C} 9 \mathrm{D}-\mathrm{H} 9 \mathrm{D}$ & 119.0 \\
\hline $\mathrm{C} 10 \mathrm{D}-\mathrm{C} 9 \mathrm{D}-\mathrm{C} 8 \mathrm{D}$ & $122.0(7)$ \\
\hline $\mathrm{C} 10 \mathrm{D}-\mathrm{C} 9 \mathrm{D}-\mathrm{H} 9 \mathrm{D}$ & 119.0 \\
\hline $\mathrm{C} 9 \mathrm{~A}-\mathrm{C} 10 \mathrm{~A}-\mathrm{H} 10 \mathrm{~A}$ & 120.0 \\
\hline $\mathrm{C} 9 \mathrm{~A}-\mathrm{C} 10 \mathrm{~A}-\mathrm{C} 11 \mathrm{~A}$ & $120.0(5)$ \\
\hline $\mathrm{C} 11 \mathrm{~A}-\mathrm{C} 10 \mathrm{~A}-\mathrm{H} 10 \mathrm{~A}$ & 120.0 \\
\hline $\mathrm{C} 9 \mathrm{~B}-\mathrm{C} 10 \mathrm{~B}-\mathrm{H} 10 \mathrm{~B}$ & 119.8 \\
\hline $\mathrm{C} 9 \mathrm{~B}-\mathrm{C} 10 \mathrm{~B}-\mathrm{C} 11 \mathrm{~B}$ & $120.3(5)$ \\
\hline $\mathrm{C} 11 \mathrm{~B}-\mathrm{C} 10 \mathrm{~B}-\mathrm{H} 10 \mathrm{~B}$ & 119.8 \\
\hline $\mathrm{C} 9 \mathrm{C}-\mathrm{C} 10 \mathrm{C}-\mathrm{H} 10 \mathrm{C}$ & 119.4 \\
\hline $\mathrm{C} 9 \mathrm{C}-\mathrm{C} 10 \mathrm{C}-\mathrm{C} 11 \mathrm{C}$ & $121.2(7)$ \\
\hline $\mathrm{C} 11 \mathrm{C}-\mathrm{C} 10 \mathrm{C}-\mathrm{H} 10 \mathrm{C}$ & 119.4 \\
\hline $\mathrm{C} 9 \mathrm{D}-\mathrm{C} 10 \mathrm{D}-\mathrm{H} 10 \mathrm{D}$ & 120.3 \\
\hline $\mathrm{C} 9 \mathrm{D}-\mathrm{C} 10 \mathrm{D}-\mathrm{C} 11 \mathrm{D}$ & $119.4(8)$ \\
\hline C11D-C10D-H10D & 120.3 \\
\hline $\mathrm{C} 10 \mathrm{~A}-\mathrm{C} 11 \mathrm{~A}-\mathrm{H} 11 \mathrm{~A}$ & 119.8 \\
\hline $\mathrm{C} 12 \mathrm{~A}-\mathrm{C} 11 \mathrm{~A}-\mathrm{C} 10 \mathrm{~A}$ & $120.4(5)$ \\
\hline $\mathrm{C} 12 \mathrm{~A}-\mathrm{C} 11 \mathrm{~A}-\mathrm{H} 11 \mathrm{~A}$ & 119.8 \\
\hline $\mathrm{C} 10 \mathrm{~B}-\mathrm{C} 11 \mathrm{~B}-\mathrm{H} 11 \mathrm{~B}$ & 119.9 \\
\hline $\mathrm{C} 12 \mathrm{~B}-\mathrm{C} 11 \mathrm{~B}-\mathrm{C} 10 \mathrm{~B}$ & $120.1(5)$ \\
\hline $\mathrm{C} 12 \mathrm{~B}-\mathrm{C} 11 \mathrm{~B}-\mathrm{H} 11 \mathrm{~B}$ & 119.9 \\
\hline $\mathrm{C} 10 \mathrm{C}-\mathrm{C} 11 \mathrm{C}-\mathrm{H} 11 \mathrm{C}$ & 119.5 \\
\hline $\mathrm{C} 12 \mathrm{C}-\mathrm{C} 11 \mathrm{C}-\mathrm{C} 10 \mathrm{C}$ & $121.0(7)$ \\
\hline $\mathrm{C} 12 \mathrm{C}-\mathrm{C} 11 \mathrm{C}-\mathrm{H} 11 \mathrm{C}$ & 119.5 \\
\hline $\mathrm{C} 10 \mathrm{D}-\mathrm{C} 11 \mathrm{D}-\mathrm{H} 11 \mathrm{D}$ & 118.8 \\
\hline $\mathrm{C} 12 \mathrm{D}-\mathrm{C} 11 \mathrm{D}-\mathrm{C} 10 \mathrm{D}$ & $122.4(7)$ \\
\hline $\mathrm{C} 12 \mathrm{D}-\mathrm{C} 11 \mathrm{D}-\mathrm{H} 11 \mathrm{D}$ & 118.8 \\
\hline $\mathrm{C} 11 \mathrm{~A}-\mathrm{C} 12 \mathrm{~A}-\mathrm{H} 12 \mathrm{~A}$ & 119.0 \\
\hline $\mathrm{C} 11 \mathrm{~A}-\mathrm{C} 12 \mathrm{~A}-\mathrm{C} 13 \mathrm{~A}$ & $121.9(5)$ \\
\hline $\mathrm{C} 13 \mathrm{~A}-\mathrm{C} 12 \mathrm{~A}-\mathrm{H} 12 \mathrm{~A}$ & 119.0 \\
\hline $\mathrm{C} 11 \mathrm{~B}-\mathrm{C} 12 \mathrm{~B}-\mathrm{H} 12 \mathrm{~B}$ & 119.0 \\
\hline $\mathrm{C} 11 \mathrm{~B}-\mathrm{C} 12 \mathrm{~B}-\mathrm{C} 13 \mathrm{~B}$ & $122.1(5)$ \\
\hline $\mathrm{C} 13 \mathrm{~B}-\mathrm{C} 12 \mathrm{~B}-\mathrm{H} 12 \mathrm{~B}$ & 119.0 \\
\hline $\mathrm{C} 11 \mathrm{C}-\mathrm{C} 12 \mathrm{C}-\mathrm{H} 12 \mathrm{C}$ & 119.6 \\
\hline $\mathrm{C} 11 \mathrm{C}-\mathrm{C} 12 \mathrm{C}-\mathrm{C} 13 \mathrm{C}$ & $120.8(7)$ \\
\hline $\mathrm{C} 13 \mathrm{C}-\mathrm{C} 12 \mathrm{C}-\mathrm{H} 12 \mathrm{C}$ & 119.6 \\
\hline $\mathrm{C} 11 \mathrm{D}-\mathrm{C} 12 \mathrm{D}-\mathrm{H} 12 \mathrm{D}$ & 120.2 \\
\hline $\mathrm{C} 11 \mathrm{D}-\mathrm{C} 12 \mathrm{D}-\mathrm{C} 13 \mathrm{D}$ & $119.7(7)$ \\
\hline
\end{tabular}

$121.9(5)$

$114.3(5)$

$125.6(5)$

120.0

120.0

120.0

120.0

120.0

120.0

120.0

120.0

120.0

120.0

$118.2(4)$

119.0

122.6 (4)

121.1

119.6

119.2

119.7

120.6

119.7

120.0

120.1

120.0

119.6

120.7

119.6

118.8

122.3

118.8

117.0

120.5

122.5

119.5

121.0

119.5

121.5

119.5

119.1

120.0

120.0

120.0

119.5

121.0

119.5

119.9

120.3 


\begin{tabular}{|c|c|}
\hline $\mathrm{C} 13 \mathrm{D}-\mathrm{C} 12 \mathrm{D}-\mathrm{H} 12 \mathrm{D}$ & 120.2 \\
\hline $\mathrm{C} 12 \mathrm{~A}-\mathrm{C} 13 \mathrm{~A}-\mathrm{C} 8 \mathrm{~A}$ & $117.8(4)$ \\
\hline $\mathrm{C} 14 \mathrm{~A}-\mathrm{C} 13 \mathrm{~A}-\mathrm{C} 8 \mathrm{~A}$ & $119.6(4)$ \\
\hline $\mathrm{C} 14 \mathrm{~A}-\mathrm{C} 13 \mathrm{~A}-\mathrm{C} 12 \mathrm{~A}$ & $122.6(5)$ \\
\hline $\mathrm{C} 12 \mathrm{~B}-\mathrm{C} 13 \mathrm{~B}-\mathrm{C} 8 \mathrm{~B}$ & $117.7(4)$ \\
\hline $\mathrm{C} 14 \mathrm{~B}-\mathrm{C} 13 \mathrm{~B}-\mathrm{C} 8 \mathrm{~B}$ & $120.1(5)$ \\
\hline $\mathrm{C} 14 \mathrm{~B}-\mathrm{C} 13 \mathrm{~B}-\mathrm{C} 12 \mathrm{~B}$ & $122.2(4)$ \\
\hline $\mathrm{C} 12 \mathrm{C}-\mathrm{C} 13 \mathrm{C}-\mathrm{C} 8 \mathrm{C}$ & $119.4(6)$ \\
\hline $\mathrm{C} 14 \mathrm{C}-\mathrm{C} 13 \mathrm{C}-\mathrm{C} 8 \mathrm{C}$ & $119.7(5)$ \\
\hline $\mathrm{C} 14 \mathrm{C}-\mathrm{C} 13 \mathrm{C}-\mathrm{C} 12 \mathrm{C}$ & $120.9(6)$ \\
\hline $\mathrm{C} 8 \mathrm{D}-\mathrm{C} 13 \mathrm{D}-\mathrm{C} 12 \mathrm{D}$ & $118.8(7)$ \\
\hline $\mathrm{C} 14 \mathrm{D}-\mathrm{C} 13 \mathrm{D}-\mathrm{C} 8 \mathrm{D}$ & $119.4(5)$ \\
\hline $\mathrm{C} 14 \mathrm{D}-\mathrm{C} 13 \mathrm{D}-\mathrm{C} 12 \mathrm{D}$ & $121.8(6)$ \\
\hline $\mathrm{C} 13 \mathrm{~A}-\mathrm{C} 14 \mathrm{~A}-\mathrm{H} 14 \mathrm{~A}$ & 119.3 \\
\hline $\mathrm{C} 15 \mathrm{~A}-\mathrm{C} 14 \mathrm{~A}-\mathrm{C} 13 \mathrm{~A}$ & $121.4(4)$ \\
\hline $\mathrm{C} 15 \mathrm{~A}-\mathrm{C} 14 \mathrm{~A}-\mathrm{H} 14 \mathrm{~A}$ & 119.3 \\
\hline $\mathrm{C} 13 \mathrm{~B}-\mathrm{C} 14 \mathrm{~B}-\mathrm{H} 14 \mathrm{~B}$ & 119.2 \\
\hline $\mathrm{C} 13 \mathrm{~B}-\mathrm{C} 14 \mathrm{~B}-\mathrm{C} 15 \mathrm{~B}$ & $121.7(4)$ \\
\hline $\mathrm{C} 15 \mathrm{~B}-\mathrm{C} 14 \mathrm{~B}-\mathrm{H} 14 \mathrm{~B}$ & 119.2 \\
\hline $\mathrm{C} 13 \mathrm{C}-\mathrm{C} 14 \mathrm{C}-\mathrm{H} 14 \mathrm{C}$ & 119.0 \\
\hline $\mathrm{C} 13 \mathrm{C}-\mathrm{C} 14 \mathrm{C}-\mathrm{C} 15 \mathrm{C}$ & $121.9(5)$ \\
\hline $\mathrm{C} 15 \mathrm{C}-\mathrm{C} 14 \mathrm{C}-\mathrm{H} 14 \mathrm{C}$ & 119.0 \\
\hline $\mathrm{C} 13 \mathrm{D}-\mathrm{C} 14 \mathrm{D}-\mathrm{H} 14 \mathrm{D}$ & 118.8 \\
\hline $\mathrm{C} 13 \mathrm{D}-\mathrm{C} 14 \mathrm{D}-\mathrm{C} 15 \mathrm{D}$ & $122.4(5)$ \\
\hline $\mathrm{C} 15 \mathrm{D}-\mathrm{C} 14 \mathrm{D}-\mathrm{H} 14 \mathrm{D}$ & 118.8 \\
\hline $\mathrm{C} 14 \mathrm{~A}-\mathrm{C} 15 \mathrm{~A}-\mathrm{C} 16 \mathrm{~A}$ & $121.8(4)$ \\
\hline $\mathrm{C} 14 \mathrm{~A}-\mathrm{C} 15 \mathrm{~A}-\mathrm{C} 20 \mathrm{~A}$ & $119.3(4)$ \\
\hline $\mathrm{C} 16 \mathrm{~A}-\mathrm{C} 15 \mathrm{~A}-\mathrm{C} 20 \mathrm{~A}$ & $118.9(4)$ \\
\hline $\mathrm{C} 14 \mathrm{~B}-\mathrm{C} 15 \mathrm{~B}-\mathrm{C} 16 \mathrm{~B}$ & $122.3(5)$ \\
\hline $\mathrm{C} 14 \mathrm{~B}-\mathrm{C} 15 \mathrm{~B}-\mathrm{C} 20 \mathrm{~B}$ & $119.0(4)$ \\
\hline $\mathrm{C} 20 \mathrm{~B}-\mathrm{C} 15 \mathrm{~B}-\mathrm{C} 16 \mathrm{~B}$ & $118.6(5)$ \\
\hline $\mathrm{C} 14 \mathrm{C}-\mathrm{C} 15 \mathrm{C}-\mathrm{C} 16 \mathrm{C}$ & $122.1(5)$ \\
\hline $\mathrm{C} 14 \mathrm{C}-\mathrm{C} 15 \mathrm{C}-\mathrm{C} 20 \mathrm{C}$ & $119.5(5)$ \\
\hline $\mathrm{C} 20 \mathrm{C}-\mathrm{C} 15 \mathrm{C}-\mathrm{C} 16 \mathrm{C}$ & $118.4(5)$ \\
\hline $\mathrm{C} 14 \mathrm{D}-\mathrm{C} 15 \mathrm{D}-\mathrm{C} 16 \mathrm{D}$ & $122.8(5)$ \\
\hline $\mathrm{C} 14 \mathrm{D}-\mathrm{C} 15 \mathrm{D}-\mathrm{C} 20 \mathrm{D}$ & $118.2(6)$ \\
\hline $\mathrm{C} 16 \mathrm{D}-\mathrm{C} 15 \mathrm{D}-\mathrm{C} 20 \mathrm{D}$ & $119.0(5)$ \\
\hline $\mathrm{C} 15 \mathrm{~A}-\mathrm{C} 16 \mathrm{~A}-\mathrm{H} 16 \mathrm{~A}$ & 119.6 \\
\hline $\mathrm{C} 17 \mathrm{~A}-\mathrm{C} 16 \mathrm{~A}-\mathrm{C} 15 \mathrm{~A}$ & $120.8(5)$ \\
\hline $\mathrm{C} 17 \mathrm{~A}-\mathrm{C} 16 \mathrm{~A}-\mathrm{H} 16 \mathrm{~A}$ & 119.6 \\
\hline $\mathrm{C} 15 \mathrm{~B}-\mathrm{C} 16 \mathrm{~B}-\mathrm{H} 16 \mathrm{~B}$ & 119.2 \\
\hline $\mathrm{C} 17 \mathrm{~B}-\mathrm{C} 16 \mathrm{~B}-\mathrm{C} 15 \mathrm{~B}$ & $121.6(5)$ \\
\hline $\mathrm{C} 17 \mathrm{~B}-\mathrm{C} 16 \mathrm{~B}-\mathrm{H} 16 \mathrm{~B}$ & 119.2 \\
\hline $\mathrm{C} 15 \mathrm{C}-\mathrm{C} 16 \mathrm{C}-\mathrm{H} 16 \mathrm{C}$ & 119.4 \\
\hline $\mathrm{C} 17 \mathrm{C}-\mathrm{C} 16 \mathrm{C}-\mathrm{C} 15 \mathrm{C}$ & $121.1(5)$ \\
\hline $\mathrm{C} 17 \mathrm{C}-\mathrm{C} 16 \mathrm{C}-\mathrm{H} 16 \mathrm{C}$ & 119.4 \\
\hline $\mathrm{C} 15 \mathrm{D}-\mathrm{C} 16 \mathrm{D}-\mathrm{H} 16 \mathrm{D}$ & 119.1 \\
\hline $\mathrm{C} 17 \mathrm{D}-\mathrm{C} 16 \mathrm{D}-\mathrm{C} 15 \mathrm{D}$ & $121.9(5)$ \\
\hline
\end{tabular}

\begin{tabular}{|c|c|}
\hline $\mathrm{C} 19 \mathrm{~F}-\mathrm{C} 18 \mathrm{~F}-\mathrm{H} 18 \mathrm{~F}$ & 119.9 \\
\hline $\mathrm{C} 18 \mathrm{~F}-\mathrm{C} 19 \mathrm{~F}-\mathrm{H} 19 \mathrm{~F}$ & 120.0 \\
\hline $\mathrm{C} 18 \mathrm{~F}-\mathrm{C} 19 \mathrm{~F}-\mathrm{C} 20 \mathrm{~F}$ & 120.0 \\
\hline $\mathrm{C} 20 \mathrm{~F}-\mathrm{C} 19 \mathrm{~F}-\mathrm{H} 19 \mathrm{~F}$ & 120.0 \\
\hline $\mathrm{C} 7 \mathrm{~F}-\mathrm{C} 20 \mathrm{~F}-\mathrm{C} 19 \mathrm{~F}$ & 120.4 \\
\hline $\mathrm{C} 15 \mathrm{~F}-\mathrm{C} 20 \mathrm{~F}-\mathrm{C} 7 \mathrm{~F}$ & 120.0 \\
\hline $\mathrm{C} 15 \mathrm{~F}-\mathrm{C} 20 \mathrm{~F}-\mathrm{C} 19 \mathrm{~F}$ & 119.5 \\
\hline $\mathrm{O} 6 \mathrm{~B}-\mathrm{C} 21 \mathrm{~B}-\mathrm{H} 21 \mathrm{C}$ & 110.8 \\
\hline $\mathrm{O} 6 \mathrm{~B}-\mathrm{C} 21 \mathrm{~B}-\mathrm{H} 21 \mathrm{D}$ & 110.8 \\
\hline $\mathrm{O} 6 \mathrm{~B}-\mathrm{C} 21 \mathrm{~B}-\mathrm{C} 22 \mathrm{~B}$ & $104.7(15)$ \\
\hline $\mathrm{H} 21 \mathrm{C}-\mathrm{C} 21 \mathrm{~B}-\mathrm{H} 21 \mathrm{D}$ & 108.9 \\
\hline $\mathrm{C} 22 \mathrm{~B}-\mathrm{C} 21 \mathrm{~B}-\mathrm{H} 21 \mathrm{C}$ & 110.8 \\
\hline $\mathrm{C} 22 \mathrm{~B}-\mathrm{C} 21 \mathrm{~B}-\mathrm{H} 21 \mathrm{D}$ & 110.8 \\
\hline $\mathrm{C} 21 \mathrm{~B}-\mathrm{C} 22 \mathrm{~B}-\mathrm{H} 22 \mathrm{C}$ & 111.2 \\
\hline $\mathrm{C} 21 \mathrm{~B}-\mathrm{C} 22 \mathrm{~B}-\mathrm{H} 22 \mathrm{D}$ & 111.2 \\
\hline $\mathrm{C} 21 \mathrm{~B}-\mathrm{C} 22 \mathrm{~B}-\mathrm{C} 23 \mathrm{~B}$ & $102.6(16)$ \\
\hline $\mathrm{H} 22 \mathrm{C}-\mathrm{C} 22 \mathrm{~B}-\mathrm{H} 22 \mathrm{D}$ & 109.2 \\
\hline $\mathrm{C} 23 \mathrm{~B}-\mathrm{C} 22 \mathrm{~B}-\mathrm{H} 22 \mathrm{C}$ & 111.2 \\
\hline $\mathrm{C} 23 \mathrm{~B}-\mathrm{C} 22 \mathrm{~B}-\mathrm{H} 22 \mathrm{D}$ & 111.2 \\
\hline $\mathrm{C} 22 \mathrm{~B}-\mathrm{C} 23 \mathrm{~B}-\mathrm{H} 23 \mathrm{C}$ & 110.3 \\
\hline $\mathrm{C} 22 \mathrm{~B}-\mathrm{C} 23 \mathrm{~B}-\mathrm{H} 23 \mathrm{D}$ & 110.3 \\
\hline $\mathrm{H} 23 \mathrm{C}-\mathrm{C} 23 \mathrm{~B}-\mathrm{H} 23 \mathrm{D}$ & 108.6 \\
\hline $\mathrm{C} 24 \mathrm{~B}-\mathrm{C} 23 \mathrm{~B}-\mathrm{C} 22 \mathrm{~B}$ & $106.9(13)$ \\
\hline $\mathrm{C} 24 \mathrm{~B}-\mathrm{C} 23 \mathrm{~B}-\mathrm{H} 23 \mathrm{C}$ & 110.3 \\
\hline $\mathrm{C} 24 \mathrm{~B}-\mathrm{C} 23 \mathrm{~B}-\mathrm{H} 23 \mathrm{D}$ & 110.3 \\
\hline $\mathrm{O} 6 \mathrm{~B}-\mathrm{C} 24 \mathrm{~B}-\mathrm{H} 24 \mathrm{C}$ & 110.0 \\
\hline $\mathrm{O} 6 \mathrm{~B}-\mathrm{C} 24 \mathrm{~B}-\mathrm{H} 24 \mathrm{D}$ & 110.0 \\
\hline $\mathrm{C} 23 \mathrm{~B}-\mathrm{C} 24 \mathrm{~B}-\mathrm{O} 6 \mathrm{~B}$ & $108.2(12)$ \\
\hline $\mathrm{C} 23 \mathrm{~B}-\mathrm{C} 24 \mathrm{~B}-\mathrm{H} 24 \mathrm{C}$ & 110.0 \\
\hline $\mathrm{C} 23 \mathrm{~B}-\mathrm{C} 24 \mathrm{~B}-\mathrm{H} 24 \mathrm{D}$ & 110.0 \\
\hline $\mathrm{H} 24 \mathrm{C}-\mathrm{C} 24 \mathrm{~B}-\mathrm{H} 24 \mathrm{D}$ & 108.4 \\
\hline $\mathrm{H} 29 \mathrm{D}-\mathrm{C} 29 \mathrm{~B}-\mathrm{H} 29 \mathrm{E}$ & 109.5 \\
\hline $\mathrm{H} 29 \mathrm{D}-\mathrm{C} 29 \mathrm{~B}-\mathrm{H} 29 \mathrm{~F}$ & 109.5 \\
\hline $\mathrm{H} 29 \mathrm{E}-\mathrm{C} 29 \mathrm{~B}-\mathrm{H} 29 \mathrm{~F}$ & 109.5 \\
\hline $\mathrm{C} 30 \mathrm{~B}-\mathrm{C} 29 \mathrm{~B}-\mathrm{H} 29 \mathrm{D}$ & 109.5 \\
\hline $\mathrm{C} 30 \mathrm{~B}-\mathrm{C} 29 \mathrm{~B}-\mathrm{H} 29 \mathrm{E}$ & 109.5 \\
\hline $\mathrm{C} 30 \mathrm{~B}-\mathrm{C} 29 \mathrm{~B}-\mathrm{H} 29 \mathrm{~F}$ & 109.5 \\
\hline $\mathrm{C} 31 \mathrm{~B}-\mathrm{C} 30 \mathrm{~B}-\mathrm{C} 29 \mathrm{~B}$ & 119.4 \\
\hline $\mathrm{C} 35 \mathrm{~B}-\mathrm{C} 30 \mathrm{~B}-\mathrm{C} 29 \mathrm{~B}$ & 120.5 \\
\hline $\mathrm{C} 35 \mathrm{~B}-\mathrm{C} 30 \mathrm{~B}-\mathrm{C} 31 \mathrm{~B}$ & 120.0 \\
\hline $\mathrm{C} 30 \mathrm{~B}-\mathrm{C} 31 \mathrm{~B}-\mathrm{H} 31 \mathrm{~B}$ & 120.0 \\
\hline $\mathrm{C} 32 \mathrm{~B}-\mathrm{C} 31 \mathrm{~B}-\mathrm{C} 30 \mathrm{~B}$ & 119.9 \\
\hline $\mathrm{C} 32 \mathrm{~B}-\mathrm{C} 31 \mathrm{~B}-\mathrm{H} 31 \mathrm{~B}$ & 120.0 \\
\hline $\mathrm{C} 31 \mathrm{~B}-\mathrm{C} 32 \mathrm{~B}-\mathrm{H} 32 \mathrm{~B}$ & 120.0 \\
\hline $\mathrm{C} 31 \mathrm{~B}-\mathrm{C} 32 \mathrm{~B}-\mathrm{C} 33 \mathrm{~B}$ & 120.1 \\
\hline $\mathrm{C} 33 \mathrm{~B}-\mathrm{C} 32 \mathrm{~B}-\mathrm{H} 32 \mathrm{~B}$ & 120.0 \\
\hline $\mathrm{C} 32 \mathrm{~B}-\mathrm{C} 33 \mathrm{~B}-\mathrm{H} 33 \mathrm{~B}$ & 120.0 \\
\hline $\mathrm{C} 32 \mathrm{~B}-\mathrm{C} 33 \mathrm{~B}-\mathrm{C} 34 \mathrm{~B}$ & 120.0 \\
\hline
\end{tabular}




\begin{tabular}{|c|c|}
\hline $\mathrm{C} 17 \mathrm{D}-\mathrm{C} 16 \mathrm{D}-\mathrm{H} 16 \mathrm{D}$ & 119.1 \\
\hline $\mathrm{C} 16 \mathrm{~A}-\mathrm{C} 17 \mathrm{~A}-\mathrm{H} 17 \mathrm{~A}$ & 119.6 \\
\hline $\mathrm{C} 16 \mathrm{~A}-\mathrm{C} 17 \mathrm{~A}-\mathrm{C} 18 \mathrm{~A}$ & $120.8(4)$ \\
\hline $\mathrm{C} 18 \mathrm{~A}-\mathrm{C} 17 \mathrm{~A}-\mathrm{H} 17 \mathrm{~A}$ & 119.6 \\
\hline $\mathrm{C} 16 \mathrm{~B}-\mathrm{C} 17 \mathrm{~B}-\mathrm{H} 17 \mathrm{~B}$ & 119.9 \\
\hline $\mathrm{C} 16 \mathrm{~B}-\mathrm{C} 17 \mathrm{~B}-\mathrm{C} 18 \mathrm{~B}$ & $120.2(5)$ \\
\hline $\mathrm{C} 18 \mathrm{~B}-\mathrm{C} 17 \mathrm{~B}-\mathrm{H} 17 \mathrm{~B}$ & 119.9 \\
\hline $\mathrm{C} 16 \mathrm{C}-\mathrm{C} 17 \mathrm{C}-\mathrm{H} 17 \mathrm{C}$ & 119.7 \\
\hline $\mathrm{C} 16 \mathrm{C}-\mathrm{C} 17 \mathrm{C}-\mathrm{C} 18 \mathrm{C}$ & $120.5(5)$ \\
\hline $\mathrm{C} 18 \mathrm{C}-\mathrm{C} 17 \mathrm{C}-\mathrm{H} 17 \mathrm{C}$ & 119.7 \\
\hline $\mathrm{C} 16 \mathrm{D}-\mathrm{C} 17 \mathrm{D}-\mathrm{H} 17 \mathrm{D}$ & 120.4 \\
\hline $\mathrm{C} 16 \mathrm{D}-\mathrm{C} 17 \mathrm{D}-\mathrm{C} 18 \mathrm{D}$ & $119.2(5)$ \\
\hline C18D-C17D-H17D & 120.4 \\
\hline $\mathrm{C} 17 \mathrm{~A}-\mathrm{C} 18 \mathrm{~A}-\mathrm{H} 18 \mathrm{~A}$ & 119.9 \\
\hline $\mathrm{C} 19 \mathrm{~A}-\mathrm{C} 18 \mathrm{~A}-\mathrm{C} 17 \mathrm{~A}$ & $120.3(5)$ \\
\hline $\mathrm{C} 19 \mathrm{~A}-\mathrm{C} 18 \mathrm{~A}-\mathrm{H} 18 \mathrm{~A}$ & 119.9 \\
\hline $\mathrm{C} 17 \mathrm{~B}-\mathrm{C} 18 \mathrm{~B}-\mathrm{H} 18 \mathrm{~B}$ & 120.0 \\
\hline $\mathrm{C} 19 \mathrm{~B}-\mathrm{C} 18 \mathrm{~B}-\mathrm{C} 17 \mathrm{~B}$ & $120.0(5)$ \\
\hline $\mathrm{C} 19 \mathrm{~B}-\mathrm{C} 18 \mathrm{~B}-\mathrm{H} 18 \mathrm{~B}$ & 120.0 \\
\hline $\mathrm{C} 17 \mathrm{C}-\mathrm{C} 18 \mathrm{C}-\mathrm{H} 18 \mathrm{C}$ & 119.7 \\
\hline $\mathrm{C} 19 \mathrm{C}-\mathrm{C} 18 \mathrm{C}-\mathrm{C} 17 \mathrm{C}$ & $120.6(5)$ \\
\hline $\mathrm{C} 19 \mathrm{C}-\mathrm{C} 18 \mathrm{C}-\mathrm{H} 18 \mathrm{C}$ & 119.7 \\
\hline $\mathrm{C} 17 \mathrm{D}-\mathrm{C} 18 \mathrm{D}-\mathrm{H} 18 \mathrm{D}$ & 119.8 \\
\hline $\mathrm{C} 19 \mathrm{D}-\mathrm{C} 18 \mathrm{D}-\mathrm{C} 17 \mathrm{D}$ & $120.4(5)$ \\
\hline C19D-C18D-H18D & 119.8 \\
\hline $\mathrm{C} 18 \mathrm{~A}-\mathrm{C} 19 \mathrm{~A}-\mathrm{H} 19 \mathrm{~A}$ & 119.6 \\
\hline $\mathrm{C} 18 \mathrm{~A}-\mathrm{C} 19 \mathrm{~A}-\mathrm{C} 20 \mathrm{~A}$ & $120.9(5)$ \\
\hline $\mathrm{C} 20 \mathrm{~A}-\mathrm{C} 19 \mathrm{~A}-\mathrm{H} 19 \mathrm{~A}$ & 119.6 \\
\hline $\mathrm{C} 18 \mathrm{~B}-\mathrm{C} 19 \mathrm{~B}-\mathrm{H} 19 \mathrm{~B}$ & 119.0 \\
\hline $\mathrm{C} 18 \mathrm{~B}-\mathrm{C} 19 \mathrm{~B}-\mathrm{C} 20 \mathrm{~B}$ & $122.0(5)$ \\
\hline $\mathrm{C} 20 \mathrm{~B}-\mathrm{C} 19 \mathrm{~B}-\mathrm{H} 19 \mathrm{~B}$ & 119.0 \\
\hline $\mathrm{C} 18 \mathrm{C}-\mathrm{C} 19 \mathrm{C}-\mathrm{H} 19 \mathrm{C}$ & 119.4 \\
\hline $\mathrm{C} 18 \mathrm{C}-\mathrm{C} 19 \mathrm{C}-\mathrm{C} 20 \mathrm{C}$ & $121.2(5)$ \\
\hline $\mathrm{C} 20 \mathrm{C}-\mathrm{C} 19 \mathrm{C}-\mathrm{H} 19 \mathrm{C}$ & 119.4 \\
\hline $\mathrm{C} 18 \mathrm{D}-\mathrm{C} 19 \mathrm{D}-\mathrm{H} 19 \mathrm{D}$ & 119.3 \\
\hline $\mathrm{C} 18 \mathrm{D}-\mathrm{C} 19 \mathrm{D}-\mathrm{C} 20 \mathrm{D}$ & $121.4(5)$ \\
\hline C20D-C19D-H19D & 119.3 \\
\hline $\mathrm{C} 7 \mathrm{~A}-\mathrm{C} 20 \mathrm{~A}-\mathrm{C} 15 \mathrm{~A}$ & $120.1(4)$ \\
\hline $\mathrm{C} 7 \mathrm{~A}-\mathrm{C} 20 \mathrm{~A}-\mathrm{C} 19 \mathrm{~A}$ & $121.7(4)$ \\
\hline $\mathrm{C} 19 \mathrm{~A}-\mathrm{C} 20 \mathrm{~A}-\mathrm{C} 15 \mathrm{~A}$ & $118.2(4)$ \\
\hline $\mathrm{C} 7 \mathrm{~B}-\mathrm{C} 20 \mathrm{~B}-\mathrm{C} 15 \mathrm{~B}$ & $120.3(4)$ \\
\hline $\mathrm{C} 7 \mathrm{~B}-\mathrm{C} 20 \mathrm{~B}-\mathrm{C} 19 \mathrm{~B}$ & $122.1(4)$ \\
\hline $\mathrm{C} 19 \mathrm{~B}-\mathrm{C} 20 \mathrm{~B}-\mathrm{C} 15 \mathrm{~B}$ & $117.6(4)$ \\
\hline $\mathrm{C} 7 \mathrm{C}-\mathrm{C} 20 \mathrm{C}-\mathrm{C} 15 \mathrm{C}$ & $120.4(5)$ \\
\hline $\mathrm{C} 7 \mathrm{C}-\mathrm{C} 20 \mathrm{C}-\mathrm{C} 19 \mathrm{C}$ & $121.4(4)$ \\
\hline $\mathrm{C} 15 \mathrm{C}-\mathrm{C} 20 \mathrm{C}-\mathrm{C} 19 \mathrm{C}$ & $118.2(4)$ \\
\hline $\mathrm{C} 7 \mathrm{D}-\mathrm{C} 20 \mathrm{D}-\mathrm{C} 15 \mathrm{D}$ & $120.4(5)$ \\
\hline$C 7 D-C 20 D-C 19 D$ & $121.5(4)$ \\
\hline
\end{tabular}

\begin{tabular}{|c|c|}
\hline $\mathrm{C} 34 \mathrm{~B}-\mathrm{C} 33 \mathrm{~B}-\mathrm{H} 33 \mathrm{~B}$ & 120.0 \\
\hline $\mathrm{C} 33 \mathrm{~B}-\mathrm{C} 34 \mathrm{~B}-\mathrm{H} 34 \mathrm{~B}$ & 120.0 \\
\hline $\mathrm{C} 35 \mathrm{~B}-\mathrm{C} 34 \mathrm{~B}-\mathrm{C} 33 \mathrm{~B}$ & 120.0 \\
\hline $\mathrm{C} 35 \mathrm{~B}-\mathrm{C} 34 \mathrm{~B}-\mathrm{H} 34 \mathrm{~B}$ & 120.0 \\
\hline $\mathrm{C} 30 \mathrm{~B}-\mathrm{C} 35 \mathrm{~B}-\mathrm{H} 35 \mathrm{~B}$ & 120.0 \\
\hline $\mathrm{C} 34 \mathrm{~B}-\mathrm{C} 35 \mathrm{~B}-\mathrm{C} 30 \mathrm{~B}$ & 120.0 \\
\hline $\mathrm{C} 34 \mathrm{~B}-\mathrm{C} 35 \mathrm{~B}-\mathrm{H} 35 \mathrm{~B}$ & 120.0 \\
\hline $\mathrm{H} 43 \mathrm{D}-\mathrm{C} 43 \mathrm{~B}-\mathrm{H} 43 \mathrm{E}$ & 109.5 \\
\hline $\mathrm{H} 43 \mathrm{D}-\mathrm{C} 43 \mathrm{~B}-\mathrm{H} 43 \mathrm{~F}$ & 109.5 \\
\hline $\mathrm{H} 43 \mathrm{E}-\mathrm{C} 43 \mathrm{~B}-\mathrm{H} 43 \mathrm{~F}$ & 109.5 \\
\hline $\mathrm{C} 44 \mathrm{~B}-\mathrm{C} 43 \mathrm{~B}-\mathrm{H} 43 \mathrm{D}$ & 109.5 \\
\hline $\mathrm{C} 44 \mathrm{~B}-\mathrm{C} 43 \mathrm{~B}-\mathrm{H} 43 \mathrm{E}$ & 109.5 \\
\hline $\mathrm{C} 44 \mathrm{~B}-\mathrm{C} 43 \mathrm{~B}-\mathrm{H} 43 \mathrm{~F}$ & 109.5 \\
\hline $\mathrm{C} 45 \mathrm{~B}-\mathrm{C} 44 \mathrm{~B}-\mathrm{C} 43 \mathrm{~B}$ & 119.4 \\
\hline $\mathrm{C} 49 \mathrm{~B}-\mathrm{C} 44 \mathrm{~B}-\mathrm{C} 43 \mathrm{~B}$ & 120.5 \\
\hline $\mathrm{C} 49 \mathrm{~B}-\mathrm{C} 44 \mathrm{~B}-\mathrm{C} 45 \mathrm{~B}$ & 120.0 \\
\hline $\mathrm{C} 44 \mathrm{~B}-\mathrm{C} 45 \mathrm{~B}-\mathrm{H} 45 \mathrm{~B}$ & 120.0 \\
\hline $\mathrm{C} 46 \mathrm{~B}-\mathrm{C} 45 \mathrm{~B}-\mathrm{C} 44 \mathrm{~B}$ & 120.0 \\
\hline $\mathrm{C} 46 \mathrm{~B}-\mathrm{C} 45 \mathrm{~B}-\mathrm{H} 45 \mathrm{~B}$ & 120.0 \\
\hline $\mathrm{C} 45 \mathrm{~B}-\mathrm{C} 46 \mathrm{~B}-\mathrm{H} 46 \mathrm{~B}$ & 120.0 \\
\hline $\mathrm{C} 45 \mathrm{~B}-\mathrm{C} 46 \mathrm{~B}-\mathrm{C} 47 \mathrm{~B}$ & 120.0 \\
\hline $\mathrm{C} 47 \mathrm{~B}-\mathrm{C} 46 \mathrm{~B}-\mathrm{H} 46 \mathrm{~B}$ & 120.0 \\
\hline $\mathrm{C} 46 \mathrm{~B}-\mathrm{C} 47 \mathrm{~B}-\mathrm{H} 47 \mathrm{~B}$ & 120.0 \\
\hline $\mathrm{C} 48 \mathrm{~B}-\mathrm{C} 47 \mathrm{~B}-\mathrm{C} 46 \mathrm{~B}$ & 120.0 \\
\hline $\mathrm{C} 48 \mathrm{~B}-\mathrm{C} 47 \mathrm{~B}-\mathrm{H} 47 \mathrm{~B}$ & 120.0 \\
\hline $\mathrm{C} 47 \mathrm{~B}-\mathrm{C} 48 \mathrm{~B}-\mathrm{H} 48 \mathrm{~B}$ & 120.0 \\
\hline $\mathrm{C} 47 \mathrm{~B}-\mathrm{C} 48 \mathrm{~B}-\mathrm{C} 49 \mathrm{~B}$ & 119.9 \\
\hline $\mathrm{C} 49 \mathrm{~B}-\mathrm{C} 48 \mathrm{~B}-\mathrm{H} 48 \mathrm{~B}$ & 120.0 \\
\hline $\mathrm{C} 44 \mathrm{~B}-\mathrm{C} 49 \mathrm{~B}-\mathrm{C} 48 \mathrm{~B}$ & 120.1 \\
\hline $\mathrm{C} 44 \mathrm{~B}-\mathrm{C} 49 \mathrm{~B}-\mathrm{H} 49 \mathrm{~B}$ & 120.0 \\
\hline $\mathrm{C} 48 \mathrm{~B}-\mathrm{C} 49 \mathrm{~B}-\mathrm{H} 49 \mathrm{~B}$ & 120.0 \\
\hline $\mathrm{H} 36 \mathrm{~A}-\mathrm{C} 36 \mathrm{~B}-\mathrm{H} 36 \mathrm{~B}$ & 109.5 \\
\hline $\mathrm{H} 36 \mathrm{~A}-\mathrm{C} 36 \mathrm{~B}-\mathrm{H} 36 \mathrm{C}$ & 109.5 \\
\hline $\mathrm{H} 36 \mathrm{~B}-\mathrm{C} 36 \mathrm{~B}-\mathrm{H} 36 \mathrm{C}$ & 109.5 \\
\hline $\mathrm{C} 37 \mathrm{~B}-\mathrm{C} 36 \mathrm{~B}-\mathrm{H} 36 \mathrm{~A}$ & 109.5 \\
\hline $\mathrm{C} 37 \mathrm{~B}-\mathrm{C} 36 \mathrm{~B}-\mathrm{H} 36 \mathrm{~B}$ & 109.5 \\
\hline $\mathrm{C} 37 \mathrm{~B}-\mathrm{C} 36 \mathrm{~B}-\mathrm{H} 36 \mathrm{C}$ & 109.5 \\
\hline $\mathrm{C} 38 \mathrm{~B}-\mathrm{C} 37 \mathrm{~B}-\mathrm{C} 36 \mathrm{~B}$ & 119.3 \\
\hline $\mathrm{C} 42 \mathrm{~B}-\mathrm{C} 37 \mathrm{~B}-\mathrm{C} 36 \mathrm{~B}$ & 120.6 \\
\hline $\mathrm{C} 42 \mathrm{~B}-\mathrm{C} 37 \mathrm{~B}-\mathrm{C} 38 \mathrm{~B}$ & 120.0 \\
\hline $\mathrm{C} 37 \mathrm{~B}-\mathrm{C} 38 \mathrm{~B}-\mathrm{H} 38 \mathrm{~B}$ & 120.0 \\
\hline $\mathrm{C} 39 \mathrm{~B}-\mathrm{C} 38 \mathrm{~B}-\mathrm{C} 37 \mathrm{~B}$ & 119.9 \\
\hline $\mathrm{C} 39 \mathrm{~B}-\mathrm{C} 38 \mathrm{~B}-\mathrm{H} 38 \mathrm{~B}$ & 120.0 \\
\hline $\mathrm{C} 38 \mathrm{~B}-\mathrm{C} 39 \mathrm{~B}-\mathrm{H} 39 \mathrm{~B}$ & 120.0 \\
\hline $\mathrm{C} 38 \mathrm{~B}-\mathrm{C} 39 \mathrm{~B}-\mathrm{C} 40 \mathrm{~B}$ & 120.0 \\
\hline $\mathrm{C} 40 \mathrm{~B}-\mathrm{C} 39 \mathrm{~B}-\mathrm{H} 39 \mathrm{~B}$ & 120.0 \\
\hline $\mathrm{C} 39 \mathrm{~B}-\mathrm{C} 40 \mathrm{~B}-\mathrm{H} 40 \mathrm{~B}$ & 120.0 \\
\hline $\mathrm{C} 41 \mathrm{~B}-\mathrm{C} 40 \mathrm{~B}-\mathrm{C} 39 \mathrm{~B}$ & 120.0 \\
\hline
\end{tabular}




\begin{tabular}{|c|c|c|c|}
\hline C15D-C20D-C19D & $118.1(5)$ & $\mathrm{C} 41 \mathrm{~B}-\mathrm{C} 40 \mathrm{~B}-\mathrm{H} 40 \mathrm{~B}$ & 120.0 \\
\hline $\mathrm{O} 5-\mathrm{C} 25-\mathrm{H} 25 \mathrm{~A}$ & 110.5 & $\mathrm{C} 40 \mathrm{~B}-\mathrm{C} 41 \mathrm{~B}-\mathrm{H} 41 \mathrm{~B}$ & 120.0 \\
\hline $\mathrm{O} 5-\mathrm{C} 25-\mathrm{H} 25 \mathrm{~B}$ & 110.5 & $\mathrm{C} 40 \mathrm{~B}-\mathrm{C} 41 \mathrm{~B}-\mathrm{C} 42 \mathrm{~B}$ & 119.9 \\
\hline $\mathrm{O} 5-\mathrm{C} 25-\mathrm{C} 26$ & $106.4(4)$ & $\mathrm{C} 42 \mathrm{~B}-\mathrm{C} 41 \mathrm{~B}-\mathrm{H} 41 \mathrm{~B}$ & 120.0 \\
\hline $\mathrm{H} 25 \mathrm{~A}-\mathrm{C} 25-\mathrm{H} 25 \mathrm{~B}$ & 108.6 & $\mathrm{C} 37 \mathrm{~B}-\mathrm{C} 42 \mathrm{~B}-\mathrm{C} 41 \mathrm{~B}$ & 120.1 \\
\hline $\mathrm{C} 26-\mathrm{C} 25-\mathrm{H} 25 \mathrm{~A}$ & 110.5 & $\mathrm{C} 37 \mathrm{~B}-\mathrm{C} 42 \mathrm{~B}-\mathrm{H} 42 \mathrm{~B}$ & 120.0 \\
\hline $\mathrm{C} 26-\mathrm{C} 25-\mathrm{H} 25 \mathrm{~B}$ & 110.5 & $\mathrm{C} 41 \mathrm{~B}-\mathrm{C} 42 \mathrm{~B}-\mathrm{H} 42 \mathrm{~B}$ & 120.0 \\
\hline $\mathrm{C} 25-\mathrm{C} 26-\mathrm{H} 26 \mathrm{~A}$ & 110.3 & $\mathrm{H} 36 \mathrm{D}-\mathrm{C} 36 \mathrm{~A}-\mathrm{H} 36 \mathrm{E}$ & 109.5 \\
\hline $\mathrm{C} 25-\mathrm{C} 26-\mathrm{H} 26 \mathrm{~B}$ & 110.3 & $\mathrm{H} 36 \mathrm{D}-\mathrm{C} 36 \mathrm{~A}-\mathrm{H} 36 \mathrm{~F}$ & 109.5 \\
\hline $\mathrm{C} 25-\mathrm{C} 26-\mathrm{C} 27$ & $107.1(4)$ & $\mathrm{H} 36 \mathrm{E}-\mathrm{C} 36 \mathrm{~A}-\mathrm{H} 36 \mathrm{~F}$ & 109.5 \\
\hline $\mathrm{H} 26 \mathrm{~A}-\mathrm{C} 26-\mathrm{H} 26 \mathrm{~B}$ & 108.6 & $\mathrm{C} 37 \mathrm{~A}-\mathrm{C} 36 \mathrm{~A}-\mathrm{H} 36 \mathrm{D}$ & 109.5 \\
\hline $\mathrm{C} 27-\mathrm{C} 26-\mathrm{H} 26 \mathrm{~A}$ & 110.3 & $\mathrm{C} 37 \mathrm{~A}-\mathrm{C} 36 \mathrm{~A}-\mathrm{H} 36 \mathrm{E}$ & 109.5 \\
\hline $\mathrm{C} 27-\mathrm{C} 26-\mathrm{H} 26 \mathrm{~B}$ & 110.3 & $\mathrm{C} 37 \mathrm{~A}-\mathrm{C} 36 \mathrm{~A}-\mathrm{H} 36 \mathrm{~F}$ & 109.5 \\
\hline $\mathrm{C} 26-\mathrm{C} 27-\mathrm{H} 27 \mathrm{~A}$ & 111.0 & $\mathrm{C} 38 \mathrm{~A}-\mathrm{C} 37 \mathrm{~A}-\mathrm{C} 36 \mathrm{~A}$ & 119.3 \\
\hline $\mathrm{C} 26-\mathrm{C} 27-\mathrm{H} 27 \mathrm{~B}$ & 111.0 & $\mathrm{C} 42 \mathrm{~A}-\mathrm{C} 37 \mathrm{~A}-\mathrm{C} 36 \mathrm{~A}$ & 120.5 \\
\hline $\mathrm{H} 27 \mathrm{~A}-\mathrm{C} 27-\mathrm{H} 27 \mathrm{~B}$ & 109.0 & $\mathrm{C} 42 \mathrm{~A}-\mathrm{C} 37 \mathrm{~A}-\mathrm{C} 38 \mathrm{~A}$ & 120.0 \\
\hline $\mathrm{C} 28-\mathrm{C} 27-\mathrm{C} 26$ & $103.9(5)$ & $\mathrm{C} 37 \mathrm{~A}-\mathrm{C} 38 \mathrm{~A}-\mathrm{H} 38 \mathrm{~A}$ & 120.0 \\
\hline $\mathrm{C} 28-\mathrm{C} 27-\mathrm{H} 27 \mathrm{~A}$ & 111.0 & $\mathrm{C} 39 \mathrm{~A}-\mathrm{C} 38 \mathrm{~A}-\mathrm{C} 37 \mathrm{~A}$ & 119.9 \\
\hline $\mathrm{C} 28-\mathrm{C} 27-\mathrm{H} 27 \mathrm{~B}$ & 111.0 & $\mathrm{C} 39 \mathrm{~A}-\mathrm{C} 38 \mathrm{~A}-\mathrm{H} 38 \mathrm{~A}$ & 120.0 \\
\hline $\mathrm{O} 5-\mathrm{C} 28-\mathrm{C} 27$ & $103.9(4)$ & $\mathrm{C} 38 \mathrm{~A}-\mathrm{C} 39 \mathrm{~A}-\mathrm{H} 39 \mathrm{~A}$ & 120.0 \\
\hline $\mathrm{O} 5-\mathrm{C} 28-\mathrm{H} 28 \mathrm{~A}$ & 111.0 & $\mathrm{C} 38 \mathrm{~A}-\mathrm{C} 39 \mathrm{~A}-\mathrm{C} 40 \mathrm{~A}$ & 120.0 \\
\hline $\mathrm{O} 5-\mathrm{C} 28-\mathrm{H} 28 \mathrm{~B}$ & 111.0 & $\mathrm{C} 40 \mathrm{~A}-\mathrm{C} 39 \mathrm{~A}-\mathrm{H} 39 \mathrm{~A}$ & 120.0 \\
\hline $\mathrm{C} 27-\mathrm{C} 28-\mathrm{H} 28 \mathrm{~A}$ & 111.0 & $\mathrm{C} 39 \mathrm{~A}-\mathrm{C} 40 \mathrm{~A}-\mathrm{H} 40 \mathrm{~A}$ & 120.0 \\
\hline $\mathrm{C} 27-\mathrm{C} 28-\mathrm{H} 28 \mathrm{~B}$ & 111.0 & $\mathrm{C} 41 \mathrm{~A}-\mathrm{C} 40 \mathrm{~A}-\mathrm{C} 39 \mathrm{~A}$ & 120.0 \\
\hline $\mathrm{H} 28 \mathrm{~A}-\mathrm{C} 28-\mathrm{H} 28 \mathrm{~B}$ & 109.0 & $\mathrm{C} 41 \mathrm{~A}-\mathrm{C} 40 \mathrm{~A}-\mathrm{H} 40 \mathrm{~A}$ & 120.0 \\
\hline $\mathrm{H} 43 \mathrm{~A}-\mathrm{C} 43 \mathrm{~A}-\mathrm{H} 43 \mathrm{~B}$ & 109.5 & $\mathrm{C} 40 \mathrm{~A}-\mathrm{C} 41 \mathrm{~A}-\mathrm{H} 41 \mathrm{~A}$ & 120.0 \\
\hline $\mathrm{H} 43 \mathrm{~A}-\mathrm{C} 43 \mathrm{~A}-\mathrm{H} 43 \mathrm{C}$ & 109.5 & $\mathrm{C} 40 \mathrm{~A}-\mathrm{C} 41 \mathrm{~A}-\mathrm{C} 42 \mathrm{~A}$ & 120.0 \\
\hline $\mathrm{H} 43 \mathrm{~B}-\mathrm{C} 43 \mathrm{~A}-\mathrm{H} 43 \mathrm{C}$ & 109.5 & $\mathrm{C} 42 \mathrm{~A}-\mathrm{C} 41 \mathrm{~A}-\mathrm{H} 41 \mathrm{~A}$ & 120.0 \\
\hline $\mathrm{C} 44 \mathrm{~A}-\mathrm{C} 43 \mathrm{~A}-\mathrm{H} 43 \mathrm{~A}$ & 109.5 & $\mathrm{C} 37 \mathrm{~A}-\mathrm{C} 42 \mathrm{~A}-\mathrm{C} 41 \mathrm{~A}$ & 120.1 \\
\hline $\mathrm{C} 44 \mathrm{~A}-\mathrm{C} 43 \mathrm{~A}-\mathrm{H} 43 \mathrm{~B}$ & 109.5 & $\mathrm{C} 37 \mathrm{~A}-\mathrm{C} 42 \mathrm{~A}-\mathrm{H} 42 \mathrm{~A}$ & 120.0 \\
\hline $\mathrm{C} 44 \mathrm{~A}-\mathrm{C} 43 \mathrm{~A}-\mathrm{H} 43 \mathrm{C}$ & 109.5 & $\mathrm{C} 41 \mathrm{~A}-\mathrm{C} 42 \mathrm{~A}-\mathrm{H} 42 \mathrm{~A}$ & 120.0 \\
\hline $\mathrm{Yb} 1-\mathrm{O} 1-\mathrm{C} 1 \mathrm{D}-\mathrm{C} 2 \mathrm{D}$ & $51.1(6)$ & $\mathrm{C} 16 \mathrm{~A}-\mathrm{C} 15 \mathrm{~A}-\mathrm{C} 20 \mathrm{~A}-\mathrm{C} 19 \mathrm{~A}$ & $-4.1(6)$ \\
\hline $\mathrm{Yb} 1-\mathrm{O} 1-\mathrm{C} 1 \mathrm{D}-\mathrm{C} 6 \mathrm{D}$ & $-131.7(4)$ & $\mathrm{C} 16 \mathrm{~A}-\mathrm{C} 17 \mathrm{~A}-\mathrm{C} 18 \mathrm{~A}-\mathrm{C} 19 \mathrm{~A}$ & $-2.4(7)$ \\
\hline $\mathrm{Yb} 1-\mathrm{O} 2-\mathrm{C} 1 \mathrm{~B}-\mathrm{C} 2 \mathrm{~B}$ & $46.8(5)$ & $\mathrm{C} 16 \mathrm{~B}-\mathrm{C} 15 \mathrm{~B}-\mathrm{C} 20 \mathrm{~B}-\mathrm{C} 7 \mathrm{~B}$ & $-179.6(4)$ \\
\hline $\mathrm{Yb} 1-\mathrm{O} 2-\mathrm{C} 1 \mathrm{~B}-\mathrm{C} 6 \mathrm{~B}$ & $-134.9(4)$ & $\mathrm{C} 16 \mathrm{~B}-\mathrm{C} 15 \mathrm{~B}-\mathrm{C} 20 \mathrm{~B}-\mathrm{C} 19 \mathrm{~B}$ & $1.5(7)$ \\
\hline $\mathrm{Yb} 1-\mathrm{O} 3-\mathrm{C} 1 \mathrm{~A}-\mathrm{C} 2 \mathrm{~A}$ & $-10.8(12)$ & $\mathrm{C} 16 \mathrm{~B}-\mathrm{C} 17 \mathrm{~B}-\mathrm{C} 18 \mathrm{~B}-\mathrm{C} 19 \mathrm{~B}$ & $1.4(8)$ \\
\hline $\mathrm{Yb} 1-\mathrm{O} 3-\mathrm{C} 1 \mathrm{~A}-\mathrm{C} 6 \mathrm{~A}$ & $169.0(7)$ & $\mathrm{C} 16 \mathrm{C}-\mathrm{C} 15 \mathrm{C}-\mathrm{C} 20 \mathrm{C}-\mathrm{C} 7 \mathrm{C}$ & $179.3(4)$ \\
\hline $\mathrm{Yb} 1-\mathrm{O} 5-\mathrm{C} 25-\mathrm{C} 26$ & $155.3(4)$ & $\mathrm{C} 16 \mathrm{C}-\mathrm{C} 15 \mathrm{C}-\mathrm{C} 20 \mathrm{C}-\mathrm{C} 19 \mathrm{C}$ & $-0.4(6)$ \\
\hline $\mathrm{Yb} 1-\mathrm{O} 5-\mathrm{C} 28-\mathrm{C} 27$ & $-146.7(4)$ & $\mathrm{C} 16 \mathrm{C}-\mathrm{C} 17 \mathrm{C}-\mathrm{C} 18 \mathrm{C}-\mathrm{C} 19 \mathrm{C}$ & $0.7(7)$ \\
\hline $\mathrm{Yb} 1-\mathrm{O} 7-\mathrm{C} 1 \mathrm{E}-\mathrm{C} 2 \mathrm{E}$ & $80.0(7)$ & $\mathrm{C} 16 \mathrm{D}-\mathrm{C} 15 \mathrm{D}-\mathrm{C} 20 \mathrm{D}-\mathrm{C} 7 \mathrm{D}$ & $-177.2(4)$ \\
\hline $\mathrm{Yb} 1-\mathrm{O} 7-\mathrm{C} 1 \mathrm{E}-\mathrm{C} 6 \mathrm{E}$ & $-98.2(6)$ & $\mathrm{C} 16 \mathrm{D}-\mathrm{C} 15 \mathrm{D}-\mathrm{C} 20 \mathrm{D}-\mathrm{C} 19 \mathrm{D}$ & $3.7(6)$ \\
\hline $\mathrm{Yb} 1-\mathrm{O} 7-\mathrm{C} 1 \mathrm{~F}-\mathrm{C} 6 \mathrm{~F}$ & $-118.8(4)$ & $\mathrm{C} 16 \mathrm{D}-\mathrm{C} 17 \mathrm{D}-\mathrm{C} 18 \mathrm{D}-\mathrm{C} 19 \mathrm{D}$ & $2.9(8)$ \\
\hline $\mathrm{Yb} 1-\mathrm{O} 7-\mathrm{C} 1 \mathrm{~F}-\mathrm{C} 2 \mathrm{~F}$ & $64.8(7)$ & $\mathrm{C} 17 \mathrm{~A}-\mathrm{C} 18 \mathrm{~A}-\mathrm{C} 19 \mathrm{~A}-\mathrm{C} 20 \mathrm{~A}$ & $0.4(7)$ \\
\hline $\mathrm{Yb} 1-\mathrm{O} 6 \mathrm{~A}-\mathrm{C} 21 \mathrm{~A}-\mathrm{C} 22 \mathrm{~A}$ & $161.4(11)$ & $\mathrm{C} 17 \mathrm{~B}-\mathrm{C} 18 \mathrm{~B}-\mathrm{C} 19 \mathrm{~B}-\mathrm{C} 20 \mathrm{~B}$ & $0.2(8)$ \\
\hline $\mathrm{Yb} 1-\mathrm{O} 6 \mathrm{~A}-\mathrm{C} 24 \mathrm{~A}-\mathrm{C} 23 \mathrm{~A}$ & $-150.2(8)$ & $\mathrm{C} 17 \mathrm{C}-\mathrm{C} 18 \mathrm{C}-\mathrm{C} 19 \mathrm{C}-\mathrm{C} 20 \mathrm{C}$ & $-2.3(7)$ \\
\hline $\mathrm{Yb} 1-\mathrm{O} 6 \mathrm{~B}-\mathrm{C} 21 \mathrm{~B}-\mathrm{C} 22 \mathrm{~B}$ & $177.6(12)$ & $\mathrm{C} 17 \mathrm{D}-\mathrm{C} 18 \mathrm{D}-\mathrm{C} 19 \mathrm{D}-\mathrm{C} 20 \mathrm{D}$ & $-0.5(7)$ \\
\hline $\mathrm{Yb} 1-\mathrm{O} 6 \mathrm{~B}-\mathrm{C} 24 \mathrm{~B}-\mathrm{C} 23 \mathrm{~B}$ & $177.3(8)$ & $\mathrm{C} 18 \mathrm{~A}-\mathrm{C} 19 \mathrm{~A}-\mathrm{C} 20 \mathrm{~A}-\mathrm{C} 7 \mathrm{~A}$ & $-176.6(4)$ \\
\hline
\end{tabular}




\begin{tabular}{|c|c|}
\hline $\mathrm{Yb} 2-\mathrm{O} 1-\mathrm{C} 1 \mathrm{D}-\mathrm{C} 2 \mathrm{D}$ & $-72.2(5)$ \\
\hline $\mathrm{Yb} 2-\mathrm{O} 1-\mathrm{C} 1 \mathrm{D}-\mathrm{C} 6 \mathrm{D}$ & $104.9(4)$ \\
\hline $\mathrm{Yb} 2-\mathrm{O} 2-\mathrm{C} 1 \mathrm{~B}-\mathrm{C} 2 \mathrm{~B}$ & $-76.0(5)$ \\
\hline $\mathrm{Yb} 2-\mathrm{O} 2-\mathrm{C} 1 \mathrm{~B}-\mathrm{C} 6 \mathrm{~B}$ & $102.3(4)$ \\
\hline $\mathrm{Yb} 2-\mathrm{O} 4-\mathrm{C} 1 \mathrm{C}-\mathrm{C} 2 \mathrm{C}$ & $-40.5(16)$ \\
\hline $\mathrm{Yb} 2-\mathrm{O} 4-\mathrm{C} 1 \mathrm{C}-\mathrm{C} 6 \mathrm{C}$ & $138.5(12)$ \\
\hline $\mathrm{Yb} 2-\mathrm{O} 7-\mathrm{C} 1 \mathrm{E}-\mathrm{C} 2 \mathrm{E}$ & $-44.8(5)$ \\
\hline $\mathrm{Yb} 2-\mathrm{O} 7-\mathrm{C} 1 \mathrm{E}-\mathrm{C} 6 \mathrm{E}$ & $137.0(4)$ \\
\hline $\mathrm{Yb} 2-\mathrm{O} 7-\mathrm{C} 1 \mathrm{~F}-\mathrm{C} 6 \mathrm{~F}$ & $131.8(3)$ \\
\hline $\mathrm{Yb} 2-\mathrm{O} 7-\mathrm{C} 1 \mathrm{~F}-\mathrm{C} 2 \mathrm{~F}$ & $-44.6(6)$ \\
\hline $\mathrm{O} 1-\mathrm{C} 1 \mathrm{D}-\mathrm{C} 2 \mathrm{D}-\mathrm{C} 3 \mathrm{D}$ & $177.1(4)$ \\
\hline $\mathrm{O} 1-\mathrm{C} 1 \mathrm{D}-\mathrm{C} 6 \mathrm{D}-\mathrm{C} 5 \mathrm{D}$ & $-177.4(4)$ \\
\hline $\mathrm{O} 1-\mathrm{C} 1 \mathrm{D}-\mathrm{C} 6 \mathrm{D}-\mathrm{C} 7 \mathrm{D}$ & $2.1(7)$ \\
\hline $\mathrm{O} 2-\mathrm{C} 1 \mathrm{~B}-\mathrm{C} 2 \mathrm{~B}-\mathrm{C} 3 \mathrm{~B}$ & $-176.9(4)$ \\
\hline $\mathrm{O} 2-\mathrm{C} 1 \mathrm{~B}-\mathrm{C} 6 \mathrm{~B}-\mathrm{C} 5 \mathrm{~B}$ & $175.7(4)$ \\
\hline $\mathrm{O} 2-\mathrm{C} 1 \mathrm{~B}-\mathrm{C} 6 \mathrm{~B}-\mathrm{C} 7 \mathrm{~B}$ & $-9.3(7)$ \\
\hline $\mathrm{O} 3-\mathrm{C} 1 \mathrm{~A}-\mathrm{C} 2 \mathrm{~A}-\mathrm{C} 3 \mathrm{~A}$ & $178.9(4)$ \\
\hline $\mathrm{O} 3-\mathrm{C} 1 \mathrm{~A}-\mathrm{C} 6 \mathrm{~A}-\mathrm{C} 5 \mathrm{~A}$ & $178.2(4)$ \\
\hline $\mathrm{O} 3-\mathrm{C} 1 \mathrm{~A}-\mathrm{C} 6 \mathrm{~A}-\mathrm{C} 7 \mathrm{~A}$ & $-0.5(6)$ \\
\hline $\mathrm{O} 4-\mathrm{C} 1 \mathrm{C}-\mathrm{C} 2 \mathrm{C}-\mathrm{C} 3 \mathrm{C}$ & $179.5(5)$ \\
\hline $\mathrm{O} 4-\mathrm{C} 1 \mathrm{C}-\mathrm{C} 6 \mathrm{C}-\mathrm{C} 5 \mathrm{C}$ & $-179.3(5)$ \\
\hline $\mathrm{O} 4-\mathrm{C} 1 \mathrm{C}-\mathrm{C} 6 \mathrm{C}-\mathrm{C} 7 \mathrm{C}$ & $0.5(7)$ \\
\hline $\mathrm{O} 5-\mathrm{C} 25-\mathrm{C} 26-\mathrm{C} 27$ & $7.5(7)$ \\
\hline $\mathrm{O} 7-\mathrm{C} 1 \mathrm{E}-\mathrm{C} 2 \mathrm{E}-\mathrm{C} 3 \mathrm{E}$ & $-178.3(6)$ \\
\hline $\mathrm{O} 7-\mathrm{C} 1 \mathrm{E}-\mathrm{C} 6 \mathrm{E}-\mathrm{C} 5 \mathrm{E}$ & $178.1(7)$ \\
\hline $\mathrm{O} 7-\mathrm{C} 1 \mathrm{E}-\mathrm{C} 6 \mathrm{E}-\mathrm{C} 7 \mathrm{E}$ & $-4.7(6)$ \\
\hline $\mathrm{O} 7-\mathrm{C} 1 \mathrm{~F}-\mathrm{C} 2 \mathrm{~F}-\mathrm{C} 3 \mathrm{~F}$ & $176.3(7)$ \\
\hline $\mathrm{C} 1 \mathrm{~A}-\mathrm{C} 2 \mathrm{~A}-\mathrm{C} 3 \mathrm{~A}-\mathrm{C} 4 \mathrm{~A}$ & $3.0(7)$ \\
\hline $\mathrm{C} 1 \mathrm{~A}-\mathrm{C} 6 \mathrm{~A}-\mathrm{C} 7 \mathrm{~A}-\mathrm{C} 8 \mathrm{~A}$ & $83.9(6)$ \\
\hline $\mathrm{C} 1 \mathrm{~A}-\mathrm{C} 6 \mathrm{~A}-\mathrm{C} 7 \mathrm{~A}-\mathrm{C} 20 \mathrm{~A}$ & $-101.7(5)$ \\
\hline $\mathrm{C} 1 \mathrm{~B}-\mathrm{C} 2 \mathrm{~B}-\mathrm{C} 3 \mathrm{~B}-\mathrm{C} 4 \mathrm{~B}$ & $-0.7(8)$ \\
\hline $\mathrm{C} 1 \mathrm{~B}-\mathrm{C} 6 \mathrm{~B}-\mathrm{C} 7 \mathrm{~B}-\mathrm{C} 8 \mathrm{~B}$ & $112.4(5)$ \\
\hline $\mathrm{C} 1 \mathrm{~B}-\mathrm{C} 6 \mathrm{~B}-\mathrm{C} 7 \mathrm{~B}-\mathrm{C} 20 \mathrm{~B}$ & $-69.7(6)$ \\
\hline $\mathrm{C} 1 \mathrm{C}-\mathrm{C} 2 \mathrm{C}-\mathrm{C} 3 \mathrm{C}-\mathrm{C} 4 \mathrm{C}$ & $-0.2(8)$ \\
\hline $\mathrm{C} 1 \mathrm{C}-\mathrm{C} 6 \mathrm{C}-\mathrm{C} 7 \mathrm{C}-\mathrm{C} 8 \mathrm{C}$ & $-114.8(5)$ \\
\hline $\mathrm{C} 1 \mathrm{C}-\mathrm{C} 6 \mathrm{C}-\mathrm{C} 7 \mathrm{C}-\mathrm{C} 20 \mathrm{C}$ & $64.2(6)$ \\
\hline $\mathrm{C} 1 \mathrm{D}-\mathrm{C} 2 \mathrm{D}-\mathrm{C} 3 \mathrm{D}-\mathrm{C} 4 \mathrm{D}$ & $0.5(8)$ \\
\hline $\mathrm{C} 1 \mathrm{D}-\mathrm{C} 6 \mathrm{D}-\mathrm{C} 7 \mathrm{D}-\mathrm{C} 8 \mathrm{D}$ & $115.4(5)$ \\
\hline $\mathrm{C} 1 \mathrm{D}-\mathrm{C} 6 \mathrm{D}-\mathrm{C} 7 \mathrm{D}-\mathrm{C} 20 \mathrm{D}$ & $-63.9(6)$ \\
\hline $\mathrm{C} 2 \mathrm{~A}-\mathrm{C} 1 \mathrm{~A}-\mathrm{C} 6 \mathrm{~A}-\mathrm{C} 5 \mathrm{~A}$ & $-2.0(6)$ \\
\hline $\mathrm{C} 2 \mathrm{~A}-\mathrm{C} 1 \mathrm{~A}-\mathrm{C} 6 \mathrm{~A}-\mathrm{C} 7 \mathrm{~A}$ & $179.3(4)$ \\
\hline $\mathrm{C} 2 \mathrm{~A}-\mathrm{C} 3 \mathrm{~A}-\mathrm{C} 4 \mathrm{~A}-\mathrm{C} 5 \mathrm{~A}$ & $-2.1(7)$ \\
\hline $\mathrm{C} 2 \mathrm{~B}-\mathrm{C} 1 \mathrm{~B}-\mathrm{C} 6 \mathrm{~B}-\mathrm{C} 5 \mathrm{~B}$ & $-6.0(7)$ \\
\hline $\mathrm{C} 2 \mathrm{~B}-\mathrm{C} 1 \mathrm{~B}-\mathrm{C} 6 \mathrm{~B}-\mathrm{C} 7 \mathrm{~B}$ & $169.0(4)$ \\
\hline $\mathrm{C} 2 \mathrm{~B}-\mathrm{C} 3 \mathrm{~B}-\mathrm{C} 4 \mathrm{~B}-\mathrm{C} 5 \mathrm{~B}$ & $-1.9(8)$ \\
\hline $\mathrm{C} 2 \mathrm{C}-\mathrm{C} 1 \mathrm{C}-\mathrm{C} 6 \mathrm{C}-\mathrm{C} 5 \mathrm{C}$ & $-0.4(7)$ \\
\hline $\mathrm{C} 2 \mathrm{C}-\mathrm{C} 1 \mathrm{C}-\mathrm{C} 6 \mathrm{C}-\mathrm{C} 7 \mathrm{C}$ & $179.4(4)$ \\
\hline $\mathrm{C} 2 \mathrm{C}-\mathrm{C} 3 \mathrm{C}-\mathrm{C} 4 \mathrm{C}-\mathrm{C} 5 \mathrm{C}$ & $-0.3(9)$ \\
\hline
\end{tabular}

\begin{tabular}{|c|c|}
\hline $\mathrm{C} 18 \mathrm{~A}-\mathrm{C} 19 \mathrm{~A}-\mathrm{C} 20 \mathrm{~A}-\mathrm{C} 15 \mathrm{~A}$ & $2.8(6)$ \\
\hline $\mathrm{C} 18 \mathrm{~B}-\mathrm{C} 19 \mathrm{~B}-\mathrm{C} 20 \mathrm{~B}-\mathrm{C} 7 \mathrm{~B}$ & $179.4(5)$ \\
\hline $\mathrm{C} 18 \mathrm{~B}-\mathrm{C} 19 \mathrm{~B}-\mathrm{C} 20 \mathrm{~B}-\mathrm{C} 15 \mathrm{~B}$ & $-1.7(7)$ \\
\hline $\mathrm{C} 18 \mathrm{C}-\mathrm{C} 19 \mathrm{C}-\mathrm{C} 20 \mathrm{C}-\mathrm{C} 7 \mathrm{C}$ & $-177.6(4)$ \\
\hline $\mathrm{C} 18 \mathrm{C}-\mathrm{C} 19 \mathrm{C}-\mathrm{C} 20 \mathrm{C}-\mathrm{C} 15 \mathrm{C}$ & $2.1(6)$ \\
\hline $\mathrm{C} 18 \mathrm{D}-\mathrm{C} 19 \mathrm{D}-\mathrm{C} 20 \mathrm{D}-\mathrm{C} 7 \mathrm{D}$ & $178.2(5)$ \\
\hline $\mathrm{C} 18 \mathrm{D}-\mathrm{C} 19 \mathrm{D}-\mathrm{C} 20 \mathrm{D}-\mathrm{C} 15 \mathrm{D}$ & $-2.8(7)$ \\
\hline $\mathrm{C} 20 \mathrm{~A}-\mathrm{C} 7 \mathrm{~A}-\mathrm{C} 8 \mathrm{~A}-\mathrm{C} 9 \mathrm{~A}$ & $178.3(4)$ \\
\hline $\mathrm{C} 20 \mathrm{~A}-\mathrm{C} 7 \mathrm{~A}-\mathrm{C} 8 \mathrm{~A}-\mathrm{C} 13 \mathrm{~A}$ & $-2.5(6)$ \\
\hline $\mathrm{C} 20 \mathrm{~A}-\mathrm{C} 15 \mathrm{~A}-\mathrm{C} 16 \mathrm{~A}-\mathrm{C} 17 \mathrm{~A}$ & $2.3(7)$ \\
\hline $\mathrm{C} 20 \mathrm{~B}-\mathrm{C} 7 \mathrm{~B}-\mathrm{C} 8 \mathrm{~B}-\mathrm{C} 9 \mathrm{~B}$ & $179.4(5)$ \\
\hline $\mathrm{C} 20 \mathrm{~B}-\mathrm{C} 7 \mathrm{~B}-\mathrm{C} 8 \mathrm{~B}-\mathrm{C} 13 \mathrm{~B}$ & $0.3(7)$ \\
\hline $\mathrm{C} 20 \mathrm{~B}-\mathrm{C} 15 \mathrm{~B}-\mathrm{C} 16 \mathrm{~B}-\mathrm{C} 17 \mathrm{~B}$ & $0.1(8)$ \\
\hline $\mathrm{C} 20 \mathrm{C}-\mathrm{C} 7 \mathrm{C}-\mathrm{C} 8 \mathrm{C}-\mathrm{C} 9 \mathrm{C}$ & $-176.7(4)$ \\
\hline $\mathrm{C} 20 \mathrm{C}-\mathrm{C} 7 \mathrm{C}-\mathrm{C} 8 \mathrm{C}-\mathrm{C} 13 \mathrm{C}$ & $1.5(7)$ \\
\hline $\mathrm{C} 20 \mathrm{C}-\mathrm{C} 15 \mathrm{C}-\mathrm{C} 16 \mathrm{C}-\mathrm{C} 17 \mathrm{C}$ & $-1.1(7)$ \\
\hline $\mathrm{C} 20 \mathrm{D}-\mathrm{C} 7 \mathrm{D}-\mathrm{C} 8 \mathrm{D}-\mathrm{C} 9 \mathrm{D}$ & $179.1(5)$ \\
\hline $\mathrm{C} 20 \mathrm{D}-\mathrm{C} 7 \mathrm{D}-\mathrm{C} 8 \mathrm{D}-\mathrm{C} 13 \mathrm{D}$ & $-0.8(7)$ \\
\hline $\mathrm{C} 20 \mathrm{D}-\mathrm{C} 15 \mathrm{D}-\mathrm{C} 16 \mathrm{D}-\mathrm{C} 17 \mathrm{D}$ & $-1.4(7)$ \\
\hline $\mathrm{C} 25-\mathrm{O} 5-\mathrm{C} 28-\mathrm{C} 27$ & $36.8(5)$ \\
\hline $\mathrm{C} 25-\mathrm{C} 26-\mathrm{C} 27-\mathrm{C} 28$ & $14.6(7)$ \\
\hline $\mathrm{C} 26-\mathrm{C} 27-\mathrm{C} 28-\mathrm{O} 5$ & $-31.3(6)$ \\
\hline $\mathrm{C} 28-\mathrm{O} 5-\mathrm{C} 25-\mathrm{C} 26$ & $-27.7(6)$ \\
\hline $\mathrm{C} 43 \mathrm{~A}-\mathrm{C} 44 \mathrm{~A}-\mathrm{C} 45 \mathrm{~A}-\mathrm{C} 46 \mathrm{~A}$ & 175.6 \\
\hline $\mathrm{C} 43 \mathrm{~A}-\mathrm{C} 44 \mathrm{~A}-\mathrm{C} 49 \mathrm{~A}-\mathrm{C} 48 \mathrm{~A}$ & -175.5 \\
\hline $\mathrm{C} 44 \mathrm{~A}-\mathrm{C} 45 \mathrm{~A}-\mathrm{C} 46 \mathrm{~A}-\mathrm{C} 47 \mathrm{~A}$ & 0.1 \\
\hline $\mathrm{C} 45 \mathrm{~A}-\mathrm{C} 44 \mathrm{~A}-\mathrm{C} 49 \mathrm{~A}-\mathrm{C} 48 \mathrm{~A}$ & 0.2 \\
\hline $\mathrm{C} 45 \mathrm{~A}-\mathrm{C} 46 \mathrm{~A}-\mathrm{C} 47 \mathrm{~A}-\mathrm{C} 48 \mathrm{~A}$ & 0.0 \\
\hline $\mathrm{C} 46 \mathrm{~A}-\mathrm{C} 47 \mathrm{~A}-\mathrm{C} 48 \mathrm{~A}-\mathrm{C} 49 \mathrm{~A}$ & 0.0 \\
\hline $\mathrm{C} 47 \mathrm{~A}-\mathrm{C} 48 \mathrm{~A}-\mathrm{C} 49 \mathrm{~A}-\mathrm{C} 44 \mathrm{~A}$ & -0.2 \\
\hline $\mathrm{C} 49 \mathrm{~A}-\mathrm{C} 44 \mathrm{~A}-\mathrm{C} 45 \mathrm{~A}-\mathrm{C} 46 \mathrm{~A}$ & -0.2 \\
\hline $\mathrm{O} 6 \mathrm{~A}-\mathrm{C} 21 \mathrm{~A}-\mathrm{C} 22 \mathrm{~A}-\mathrm{C} 23 \mathrm{~A}$ & $-14.7(16)$ \\
\hline $\mathrm{C} 1 \mathrm{E}-\mathrm{C} 2 \mathrm{E}-\mathrm{C} 3 \mathrm{E}-\mathrm{C} 4 \mathrm{E}$ & 0.0 \\
\hline $\mathrm{C} 1 \mathrm{E}-\mathrm{C} 6 \mathrm{E}-\mathrm{C} 7 \mathrm{E}-\mathrm{C} 8 \mathrm{E}$ & $107.6(4)$ \\
\hline $\mathrm{C} 1 \mathrm{E}-\mathrm{C} 6 \mathrm{E}-\mathrm{C} 7 \mathrm{E}-\mathrm{C} 20 \mathrm{E}$ & $-84.8(4)$ \\
\hline $\mathrm{C} 2 \mathrm{E}-\mathrm{C} 1 \mathrm{E}-\mathrm{C} 6 \mathrm{E}-\mathrm{C} 5 \mathrm{E}$ & 0.0 \\
\hline $\mathrm{C} 2 \mathrm{E}-\mathrm{C} 1 \mathrm{E}-\mathrm{C} 6 \mathrm{E}-\mathrm{C} 7 \mathrm{E}$ & $177.2(6)$ \\
\hline $\mathrm{C} 2 \mathrm{E}-\mathrm{C} 3 \mathrm{E}-\mathrm{C} 4 \mathrm{E}-\mathrm{C} 5 \mathrm{E}$ & 0.0 \\
\hline $\mathrm{C} 3 \mathrm{E}-\mathrm{C} 4 \mathrm{E}-\mathrm{C} 5 \mathrm{E}-\mathrm{C} 6 \mathrm{E}$ & 0.0 \\
\hline $\mathrm{C} 4 \mathrm{E}-\mathrm{C} 5 \mathrm{E}-\mathrm{C} 6 \mathrm{E}-\mathrm{C} 1 \mathrm{E}$ & 0.0 \\
\hline $\mathrm{C} 4 \mathrm{E}-\mathrm{C} 5 \mathrm{E}-\mathrm{C} 6 \mathrm{E}-\mathrm{C} 7 \mathrm{E}$ & $-177.1(6)$ \\
\hline $\mathrm{C} 5 \mathrm{E}-\mathrm{C} 6 \mathrm{E}-\mathrm{C} 7 \mathrm{E}-\mathrm{C} 8 \mathrm{E}$ & $-75.2(5)$ \\
\hline $\mathrm{C} 5 \mathrm{E}-\mathrm{C} 6 \mathrm{E}-\mathrm{C} 7 \mathrm{E}-\mathrm{C} 20 \mathrm{E}$ & $92.3(5)$ \\
\hline $\mathrm{C} 6 \mathrm{E}-\mathrm{C} 1 \mathrm{E}-\mathrm{C} 2 \mathrm{E}-\mathrm{C} 3 \mathrm{E}$ & 0.0 \\
\hline $\mathrm{C} 6 \mathrm{E}-\mathrm{C} 7 \mathrm{E}-\mathrm{C} 8 \mathrm{E}-\mathrm{C} 9 \mathrm{E}$ & $-8.1(4)$ \\
\hline $\mathrm{C} 6 \mathrm{E}-\mathrm{C} 7 \mathrm{E}-\mathrm{C} 8 \mathrm{E}-\mathrm{C} 13 \mathrm{E}$ & $173.3(4)$ \\
\hline $\mathrm{C} 6 \mathrm{E}-\mathrm{C} 7 \mathrm{E}-\mathrm{C} 20 \mathrm{E}-\mathrm{C} 15 \mathrm{E}$ & $-170.8(4)$ \\
\hline $\mathrm{C} 6 \mathrm{E}-\mathrm{C} 7 \mathrm{E}-\mathrm{C} 20 \mathrm{E}-\mathrm{C} 19 \mathrm{E}$ & $10.3(4)$ \\
\hline
\end{tabular}




\begin{tabular}{|c|c|}
\hline $\mathrm{C} 2 \mathrm{D}-\mathrm{C} 1 \mathrm{D}-\mathrm{C} 6 \mathrm{D}-\mathrm{C} 5 \mathrm{D}$ & $-0.3(7)$ \\
\hline $\mathrm{C} 2 \mathrm{D}-\mathrm{C} 1 \mathrm{D}-\mathrm{C} 6 \mathrm{D}-\mathrm{C} 7 \mathrm{D}$ & $179.2(4)$ \\
\hline $\mathrm{C} 2 \mathrm{D}-\mathrm{C} 3 \mathrm{D}-\mathrm{C} 4 \mathrm{D}-\mathrm{C} 5 \mathrm{D}$ & $-0.6(8)$ \\
\hline $\mathrm{C} 3 \mathrm{~A}-\mathrm{C} 4 \mathrm{~A}-\mathrm{C} 5 \mathrm{~A}-\mathrm{C} 6 \mathrm{~A}$ & $-0.8(7)$ \\
\hline $\mathrm{C} 3 \mathrm{~B}-\mathrm{C} 4 \mathrm{~B}-\mathrm{C} 5 \mathrm{~B}-\mathrm{C} 6 \mathrm{~B}$ & $0.4(9)$ \\
\hline $\mathrm{C} 3 \mathrm{C}-\mathrm{C} 4 \mathrm{C}-\mathrm{C} 5 \mathrm{C}-\mathrm{C} 6 \mathrm{C}$ & $0.5(9)$ \\
\hline $\mathrm{C} 3 \mathrm{D}-\mathrm{C} 4 \mathrm{D}-\mathrm{C} 5 \mathrm{D}-\mathrm{C} 6 \mathrm{D}$ & $0.2(8)$ \\
\hline $\mathrm{C} 4 \mathrm{~A}-\mathrm{C} 5 \mathrm{~A}-\mathrm{C} 6 \mathrm{~A}-\mathrm{C} 1 \mathrm{~A}$ & $2.9(6)$ \\
\hline $\mathrm{C} 4 \mathrm{~A}-\mathrm{C} 5 \mathrm{~A}-\mathrm{C} 6 \mathrm{~A}-\mathrm{C} 7 \mathrm{~A}$ & $-178.4(4)$ \\
\hline $\mathrm{C} 4 \mathrm{~B}-\mathrm{C} 5 \mathrm{~B}-\mathrm{C} 6 \mathrm{~B}-\mathrm{C} 1 \mathrm{~B}$ & $3.6(8)$ \\
\hline $\mathrm{C} 4 \mathrm{~B}-\mathrm{C} 5 \mathrm{~B}-\mathrm{C} 6 \mathrm{~B}-\mathrm{C} 7 \mathrm{~B}$ & $-171.5(5)$ \\
\hline $\mathrm{C} 4 \mathrm{C}-\mathrm{C} 5 \mathrm{C}-\mathrm{C} 6 \mathrm{C}-\mathrm{C} 1 \mathrm{C}$ & $-0.2(8)$ \\
\hline $\mathrm{C} 4 \mathrm{C}-\mathrm{C} 5 \mathrm{C}-\mathrm{C} 6 \mathrm{C}-\mathrm{C} 7 \mathrm{C}$ & $-180.0(5)$ \\
\hline $\mathrm{C} 4 \mathrm{D}-\mathrm{C} 5 \mathrm{D}-\mathrm{C} 6 \mathrm{D}-\mathrm{C} 1 \mathrm{D}$ & $0.2(7)$ \\
\hline $\mathrm{C} 4 \mathrm{D}-\mathrm{C} 5 \mathrm{D}-\mathrm{C} 6 \mathrm{D}-\mathrm{C} 7 \mathrm{D}$ & $-179.3(5)$ \\
\hline $\mathrm{C} 5 \mathrm{~A}-\mathrm{C} 6 \mathrm{~A}-\mathrm{C} 7 \mathrm{~A}-\mathrm{C} 8 \mathrm{~A}$ & $-94.8(5)$ \\
\hline $\mathrm{C} 5 \mathrm{~A}-\mathrm{C} 6 \mathrm{~A}-\mathrm{C} 7 \mathrm{~A}-\mathrm{C} 20 \mathrm{~A}$ & $79.6(5)$ \\
\hline $\mathrm{C} 5 \mathrm{~B}-\mathrm{C} 6 \mathrm{~B}-\mathrm{C} 7 \mathrm{~B}-\mathrm{C} 8 \mathrm{~B}$ & $-72.7(7)$ \\
\hline $\mathrm{C} 5 \mathrm{~B}-\mathrm{C} 6 \mathrm{~B}-\mathrm{C} 7 \mathrm{~B}-\mathrm{C} 20 \mathrm{~B}$ & $105.2(5)$ \\
\hline $\mathrm{C} 5 \mathrm{C}-\mathrm{C} 6 \mathrm{C}-\mathrm{C} 7 \mathrm{C}-\mathrm{C} 8 \mathrm{C}$ & $65.0(6)$ \\
\hline $\mathrm{C} 5 \mathrm{C}-\mathrm{C} 6 \mathrm{C}-\mathrm{C} 7 \mathrm{C}-\mathrm{C} 20 \mathrm{C}$ & $-116.0(5)$ \\
\hline $\mathrm{C} 5 \mathrm{D}-\mathrm{C} 6 \mathrm{D}-\mathrm{C} 7 \mathrm{D}-\mathrm{C} 8 \mathrm{D}$ & $-65.1(6)$ \\
\hline $\mathrm{C} 5 \mathrm{D}-\mathrm{C} 6 \mathrm{D}-\mathrm{C} 7 \mathrm{D}-\mathrm{C} 20 \mathrm{D}$ & $115.6(5)$ \\
\hline $\mathrm{C} 6 \mathrm{~A}-\mathrm{C} 1 \mathrm{~A}-\mathrm{C} 2 \mathrm{~A}-\mathrm{C} 3 \mathrm{~A}$ & $-0.9(6)$ \\
\hline $\mathrm{C} 6 \mathrm{~A}-\mathrm{C} 7 \mathrm{~A}-\mathrm{C} 8 \mathrm{~A}-\mathrm{C} 9 \mathrm{~A}$ & $-7.3(7)$ \\
\hline $\mathrm{C} 6 \mathrm{~A}-\mathrm{C} 7 \mathrm{~A}-\mathrm{C} 8 \mathrm{~A}-\mathrm{C} 13 \mathrm{~A}$ & $172.0(4)$ \\
\hline $\mathrm{C} 6 \mathrm{~A}-\mathrm{C} 7 \mathrm{~A}-\mathrm{C} 20 \mathrm{~A}-\mathrm{C} 15 \mathrm{~A}$ & $-170.4(4)$ \\
\hline $\mathrm{C} 6 \mathrm{~A}-\mathrm{C} 7 \mathrm{~A}-\mathrm{C} 20 \mathrm{~A}-\mathrm{C} 19 \mathrm{~A}$ & $9.0(6)$ \\
\hline $\mathrm{C} 6 \mathrm{~B}-\mathrm{C} 1 \mathrm{~B}-\mathrm{C} 2 \mathrm{~B}-\mathrm{C} 3 \mathrm{~B}$ & $4.8(7)$ \\
\hline $\mathrm{C} 6 \mathrm{~B}-\mathrm{C} 7 \mathrm{~B}-\mathrm{C} 8 \mathrm{~B}-\mathrm{C} 9 \mathrm{~B}$ & $-2.8(7)$ \\
\hline $\mathrm{C} 6 \mathrm{~B}-\mathrm{C} 7 \mathrm{~B}-\mathrm{C} 8 \mathrm{~B}-\mathrm{C} 13 \mathrm{~B}$ & $178.2(4)$ \\
\hline $\mathrm{C} 6 \mathrm{~B}-\mathrm{C} 7 \mathrm{~B}-\mathrm{C} 20 \mathrm{~B}-\mathrm{C} 15 \mathrm{~B}$ & $-178.7(4)$ \\
\hline $\mathrm{C} 6 \mathrm{~B}-\mathrm{C} 7 \mathrm{~B}-\mathrm{C} 20 \mathrm{~B}-\mathrm{C} 19 \mathrm{~B}$ & $0.1(7)$ \\
\hline $\mathrm{C} 6 \mathrm{C}-\mathrm{C} 1 \mathrm{C}-\mathrm{C} 2 \mathrm{C}-\mathrm{C} 3 \mathrm{C}$ & $0.6(7)$ \\
\hline $\mathrm{C} 6 \mathrm{C}-\mathrm{C} 7 \mathrm{C}-\mathrm{C} 8 \mathrm{C}-\mathrm{C} 9 \mathrm{C}$ & $2.4(7)$ \\
\hline $\mathrm{C} 6 \mathrm{C}-\mathrm{C} 7 \mathrm{C}-\mathrm{C} 8 \mathrm{C}-\mathrm{C} 13 \mathrm{C}$ & $-179.5(4)$ \\
\hline $\mathrm{C} 6 \mathrm{C}-\mathrm{C} 7 \mathrm{C}-\mathrm{C} 20 \mathrm{C}-\mathrm{C} 15 \mathrm{C}$ & $178.7(4)$ \\
\hline $\mathrm{C} 6 \mathrm{C}-\mathrm{C} 7 \mathrm{C}-\mathrm{C} 20 \mathrm{C}-\mathrm{C} 19 \mathrm{C}$ & $-1.7(6)$ \\
\hline $\mathrm{C} 6 \mathrm{D}-\mathrm{C} 1 \mathrm{D}-\mathrm{C} 2 \mathrm{D}-\mathrm{C} 3 \mathrm{D}$ & $-0.1(7)$ \\
\hline $\mathrm{C} 6 \mathrm{D}-\mathrm{C} 7 \mathrm{D}-\mathrm{C} 8 \mathrm{D}-\mathrm{C} 9 \mathrm{D}$ & $-0.2(7)$ \\
\hline $\mathrm{C} 6 \mathrm{D}-\mathrm{C} 7 \mathrm{D}-\mathrm{C} 8 \mathrm{D}-\mathrm{C} 13 \mathrm{D}$ & $179.8(4)$ \\
\hline $\mathrm{C} 6 \mathrm{D}-\mathrm{C} 7 \mathrm{D}-\mathrm{C} 20 \mathrm{D}-\mathrm{C} 15 \mathrm{D}$ & $176.9(4)$ \\
\hline $\mathrm{C} 6 \mathrm{D}-\mathrm{C} 7 \mathrm{D}-\mathrm{C} 20 \mathrm{D}-\mathrm{C} 19 \mathrm{D}$ & $-4.1(7)$ \\
\hline $\mathrm{C} 7 \mathrm{~A}-\mathrm{C} 8 \mathrm{~A}-\mathrm{C} 9 \mathrm{~A}-\mathrm{C} 10 \mathrm{~A}$ & $179.4(5)$ \\
\hline $\mathrm{C} 7 \mathrm{~A}-\mathrm{C} 8 \mathrm{~A}-\mathrm{C} 13 \mathrm{~A}-\mathrm{C} 12 \mathrm{~A}$ & $179.7(5)$ \\
\hline $\mathrm{C} 7 \mathrm{~A}-\mathrm{C} 8 \mathrm{~A}-\mathrm{C} 13 \mathrm{~A}-\mathrm{C} 14 \mathrm{~A}$ & $0.4(7)$ \\
\hline $\mathrm{C} 7 \mathrm{~B}-\mathrm{C} 8 \mathrm{~B}-\mathrm{C} 9 \mathrm{~B}-\mathrm{C} 10 \mathrm{~B}$ & $-178.3(5)$ \\
\hline $\mathrm{C} 7 \mathrm{~B}-\mathrm{C} 8 \mathrm{~B}-\mathrm{C} 13 \mathrm{~B}-\mathrm{C} 12 \mathrm{~B}$ & $178.7(5)$ \\
\hline
\end{tabular}

\begin{tabular}{|c|c|}
\hline $\mathrm{C} 7 \mathrm{E}-\mathrm{C} 8 \mathrm{E}-\mathrm{C} 9 \mathrm{E}-\mathrm{C} 10 \mathrm{E}$ & -175.4 \\
\hline $\mathrm{C} 7 \mathrm{E}-\mathrm{C} 8 \mathrm{E}-\mathrm{C} 13 \mathrm{E}-\mathrm{C} 12 \mathrm{E}$ & 175.4 \\
\hline $\mathrm{C} 7 \mathrm{E}-\mathrm{C} 8 \mathrm{E}-\mathrm{C} 13 \mathrm{E}-\mathrm{C} 14 \mathrm{E}$ & -5.6 \\
\hline $\mathrm{C} 16 \mathrm{E}-\mathrm{C} 15 \mathrm{E}-\mathrm{C} 20 \mathrm{E}-\mathrm{C} 7 \mathrm{E}$ & -179.2 \\
\hline $\mathrm{C} 16 \mathrm{E}-\mathrm{C} 15 \mathrm{E}-\mathrm{C} 20 \mathrm{E}-\mathrm{C} 19 \mathrm{E}$ & -0.3 \\
\hline $\mathrm{C} 16 \mathrm{E}-\mathrm{C} 17 \mathrm{E}-\mathrm{C} 18 \mathrm{E}-\mathrm{C} 19 \mathrm{E}$ & -3.8 \\
\hline $\mathrm{C} 8 \mathrm{E}-\mathrm{C} 7 \mathrm{E}-\mathrm{C} 20 \mathrm{E}-\mathrm{C} 15 \mathrm{E}$ & -3.2 \\
\hline $\mathrm{C} 8 \mathrm{E}-\mathrm{C} 7 \mathrm{E}-\mathrm{C} 20 \mathrm{E}-\mathrm{C} 19 \mathrm{E}$ & 177.9 \\
\hline $\mathrm{C} 8 \mathrm{E}-\mathrm{C} 9 \mathrm{E}-\mathrm{C} 10 \mathrm{E}-\mathrm{C} 11 \mathrm{E}$ & -0.7 \\
\hline $\mathrm{C} 8 \mathrm{E}-\mathrm{C} 13 \mathrm{E}-\mathrm{C} 14 \mathrm{E}-\mathrm{C} 15 \mathrm{E}$ & 3.3 \\
\hline $\mathrm{C} 9 \mathrm{E}-\mathrm{C} 8 \mathrm{E}-\mathrm{C} 13 \mathrm{E}-\mathrm{C} 12 \mathrm{E}$ & -3.3 \\
\hline $\mathrm{C} 9 \mathrm{E}-\mathrm{C} 8 \mathrm{E}-\mathrm{C} 13 \mathrm{E}-\mathrm{C} 14 \mathrm{E}$ & 175.7 \\
\hline $\mathrm{C} 9 \mathrm{E}-\mathrm{C} 10 \mathrm{E}-\mathrm{C} 11 \mathrm{E}-\mathrm{C} 12 \mathrm{E}$ & -1.9 \\
\hline $\mathrm{C} 10 \mathrm{E}-\mathrm{C} 11 \mathrm{E}-\mathrm{C} 12 \mathrm{E}-\mathrm{C} 13 \mathrm{E}$ & 1.9 \\
\hline $\mathrm{C} 11 \mathrm{E}-\mathrm{C} 12 \mathrm{E}-\mathrm{C} 13 \mathrm{E}-\mathrm{C} 8 \mathrm{E}$ & 0.7 \\
\hline $\mathrm{C} 11 \mathrm{E}-\mathrm{C} 12 \mathrm{E}-\mathrm{C} 13 \mathrm{E}-\mathrm{C} 14 \mathrm{E}$ & -178.3 \\
\hline $\mathrm{C} 12 \mathrm{E}-\mathrm{C} 13 \mathrm{E}-\mathrm{C} 14 \mathrm{E}-\mathrm{C} 15 \mathrm{E}$ & -177.7 \\
\hline $\mathrm{C} 13 \mathrm{E}-\mathrm{C} 8 \mathrm{E}-\mathrm{C} 9 \mathrm{E}-\mathrm{C} 10 \mathrm{E}$ & 3.3 \\
\hline $\mathrm{C} 13 \mathrm{E}-\mathrm{C} 14 \mathrm{E}-\mathrm{C} 15 \mathrm{E}-\mathrm{C} 16 \mathrm{E}$ & 179.2 \\
\hline $\mathrm{C} 13 \mathrm{E}-\mathrm{C} 14 \mathrm{E}-\mathrm{C} 15 \mathrm{E}-\mathrm{C} 20 \mathrm{E}$ & -0.9 \\
\hline $\mathrm{C} 14 \mathrm{E}-\mathrm{C} 15 \mathrm{E}-\mathrm{C} 20 \mathrm{E}-\mathrm{C} 7 \mathrm{E}$ & 0.9 \\
\hline $\mathrm{C} 14 \mathrm{E}-\mathrm{C} 15 \mathrm{E}-\mathrm{C} 20 \mathrm{E}-\mathrm{C} 19 \mathrm{E}$ & 179.8 \\
\hline $\mathrm{C} 15 \mathrm{E}-\mathrm{C} 16 \mathrm{E}-\mathrm{C} 17 \mathrm{E}-\mathrm{C} 18 \mathrm{E}$ & 4.3 \\
\hline $\mathrm{C} 17 \mathrm{E}-\mathrm{C} 16 \mathrm{E}-\mathrm{C} 15 \mathrm{E}-\mathrm{C} 14 \mathrm{E}$ & 177.7 \\
\hline $\mathrm{C} 17 \mathrm{E}-\mathrm{C} 16 \mathrm{E}-\mathrm{C} 15 \mathrm{E}-\mathrm{C} 20 \mathrm{E}$ & -2.2 \\
\hline $\mathrm{C} 17 \mathrm{E}-\mathrm{C} 18 \mathrm{E}-\mathrm{C} 19 \mathrm{E}-\mathrm{C} 20 \mathrm{E}$ & 1.3 \\
\hline $\mathrm{C} 18 \mathrm{E}-\mathrm{C} 19 \mathrm{E}-\mathrm{C} 20 \mathrm{E}-\mathrm{C} 7 \mathrm{E}$ & 179.6 \\
\hline $\mathrm{C} 18 \mathrm{E}-\mathrm{C} 19 \mathrm{E}-\mathrm{C} 20 \mathrm{E}-\mathrm{C} 15 \mathrm{E}$ & 0.7 \\
\hline $\mathrm{C} 20 \mathrm{E}-\mathrm{C} 7 \mathrm{E}-\mathrm{C} 8 \mathrm{E}-\mathrm{C} 9 \mathrm{E}$ & -175.8 \\
\hline $\mathrm{C} 20 \mathrm{E}-\mathrm{C} 7 \mathrm{E}-\mathrm{C} 8 \mathrm{E}-\mathrm{C} 13 \mathrm{E}$ & 5.5 \\
\hline $\mathrm{C} 21 \mathrm{~A}-\mathrm{O} 6 \mathrm{~A}-\mathrm{C} 24 \mathrm{~A}-\mathrm{C} 23 \mathrm{~A}$ & $11.8(14)$ \\
\hline $\mathrm{C} 21 \mathrm{~A}-\mathrm{C} 22 \mathrm{~A}-\mathrm{C} 23 \mathrm{~A}-\mathrm{C} 24 \mathrm{~A}$ & $21.6(14)$ \\
\hline $\mathrm{C} 22 \mathrm{~A}-\mathrm{C} 23 \mathrm{~A}-\mathrm{C} 24 \mathrm{~A}-\mathrm{O} 6 \mathrm{~A}$ & $-21.8(14)$ \\
\hline $\mathrm{C} 24 \mathrm{~A}-\mathrm{O} 6 \mathrm{~A}-\mathrm{C} 21 \mathrm{~A}-\mathrm{C} 22 \mathrm{~A}$ & $2.1(14)$ \\
\hline $\mathrm{C} 29 \mathrm{~A}-\mathrm{C} 30 \mathrm{~A}-\mathrm{C} 31 \mathrm{~A}-\mathrm{C} 32 \mathrm{~A}$ & 175.6 \\
\hline $\mathrm{C} 29 \mathrm{~A}-\mathrm{C} 30 \mathrm{~A}-\mathrm{C} 35 \mathrm{~A}-\mathrm{C} 34 \mathrm{~A}$ & -175.5 \\
\hline $\mathrm{C} 30 \mathrm{~A}-\mathrm{C} 31 \mathrm{~A}-\mathrm{C} 32 \mathrm{~A}-\mathrm{C} 33 \mathrm{~A}$ & 0.1 \\
\hline $\mathrm{C} 31 \mathrm{~A}-\mathrm{C} 30 \mathrm{~A}-\mathrm{C} 35 \mathrm{~A}-\mathrm{C} 34 \mathrm{~A}$ & 0.2 \\
\hline $\mathrm{C} 31 \mathrm{~A}-\mathrm{C} 32 \mathrm{~A}-\mathrm{C} 33 \mathrm{~A}-\mathrm{C} 34 \mathrm{~A}$ & 0.1 \\
\hline $\mathrm{C} 32 \mathrm{~A}-\mathrm{C} 33 \mathrm{~A}-\mathrm{C} 34 \mathrm{~A}-\mathrm{C} 35 \mathrm{~A}$ & -0.1 \\
\hline $\mathrm{C} 33 \mathrm{~A}-\mathrm{C} 34 \mathrm{~A}-\mathrm{C} 35 \mathrm{~A}-\mathrm{C} 30 \mathrm{~A}$ & -0.1 \\
\hline $\mathrm{C} 35 \mathrm{~A}-\mathrm{C} 30 \mathrm{~A}-\mathrm{C} 31 \mathrm{~A}-\mathrm{C} 32 \mathrm{~A}$ & -0.2 \\
\hline $\mathrm{O} 6 \mathrm{~B}-\mathrm{C} 21 \mathrm{~B}-\mathrm{C} 22 \mathrm{~B}-\mathrm{C} 23 \mathrm{~B}$ & $-21.5(17)$ \\
\hline $\mathrm{C} 5 \mathrm{~F}-\mathrm{C} 6 \mathrm{~F}-\mathrm{C} 1 \mathrm{~F}-\mathrm{O} 7$ & $-176.7(7)$ \\
\hline $\mathrm{C} 5 \mathrm{~F}-\mathrm{C} 6 \mathrm{~F}-\mathrm{C} 1 \mathrm{~F}-\mathrm{C} 2 \mathrm{~F}$ & 0.0 \\
\hline $\mathrm{C} 5 \mathrm{~F}-\mathrm{C} 6 \mathrm{~F}-\mathrm{C} 7 \mathrm{~F}-\mathrm{C} 8 \mathrm{~F}$ & $102.9(5)$ \\
\hline $\mathrm{C} 5 \mathrm{~F}-\mathrm{C} 6 \mathrm{~F}-\mathrm{C} 7 \mathrm{~F}-\mathrm{C} 20 \mathrm{~F}$ & $-73.1(6)$ \\
\hline $\mathrm{C} 6 \mathrm{~F}-\mathrm{C} 5 \mathrm{~F}-\mathrm{C} 4 \mathrm{~F}-\mathrm{C} 3 \mathrm{~F}$ & 0.0 \\
\hline
\end{tabular}




\begin{tabular}{|c|c|}
\hline $\mathrm{C} 7 \mathrm{~B}-\mathrm{C} 8 \mathrm{~B}-\mathrm{C} 13 \mathrm{~B}-\mathrm{C} 14 \mathrm{~B}$ & $0.1(7)$ \\
\hline $\mathrm{C} 7 \mathrm{C}-\mathrm{C} 8 \mathrm{C}-\mathrm{C} 9 \mathrm{C}-\mathrm{C} 10 \mathrm{C}$ & $177.5(5)$ \\
\hline $\mathrm{C} 7 \mathrm{C}-\mathrm{C} 8 \mathrm{C}-\mathrm{C} 13 \mathrm{C}-\mathrm{C} 12 \mathrm{C}$ & $-178.1(5)$ \\
\hline $\mathrm{C} 7 \mathrm{C}-\mathrm{C} 8 \mathrm{C}-\mathrm{C} 13 \mathrm{C}-\mathrm{C} 14 \mathrm{C}$ & $0.0(7)$ \\
\hline $\mathrm{C} 7 \mathrm{D}-\mathrm{C} 8 \mathrm{D}-\mathrm{C} 9 \mathrm{D}-\mathrm{C} 10 \mathrm{D}$ & $179.1(5)$ \\
\hline $\mathrm{C} 7 \mathrm{D}-\mathrm{C} 8 \mathrm{D}-\mathrm{C} 13 \mathrm{D}-\mathrm{C} 12 \mathrm{D}$ & $-179.0(5)$ \\
\hline $\mathrm{C} 7 \mathrm{D}-\mathrm{C} 8 \mathrm{D}-\mathrm{C} 13 \mathrm{D}-\mathrm{C} 14 \mathrm{D}$ & $3.0(7)$ \\
\hline $\mathrm{C} 8 \mathrm{~A}-\mathrm{C} 7 \mathrm{~A}-\mathrm{C} 20 \mathrm{~A}-\mathrm{C} 15 \mathrm{~A}$ & $4.1(6)$ \\
\hline $\mathrm{C} 8 \mathrm{~A}-\mathrm{C} 7 \mathrm{~A}-\mathrm{C} 20 \mathrm{~A}-\mathrm{C} 19 \mathrm{~A}$ & $-176.5(4)$ \\
\hline $\mathrm{C} 8 \mathrm{~A}-\mathrm{C} 9 \mathrm{~A}-\mathrm{C} 10 \mathrm{~A}-\mathrm{C} 11 \mathrm{~A}$ & $1.5(8)$ \\
\hline $\mathrm{C} 8 \mathrm{~A}-\mathrm{C} 13 \mathrm{~A}-\mathrm{C} 14 \mathrm{~A}-\mathrm{C} 15 \mathrm{~A}$ & $0.1(7)$ \\
\hline $\mathrm{C} 8 \mathrm{~B}-\mathrm{C} 7 \mathrm{~B}-\mathrm{C} 20 \mathrm{~B}-\mathrm{C} 15 \mathrm{~B}$ & $-0.8(7)$ \\
\hline $\mathrm{C} 8 \mathrm{~B}-\mathrm{C} 7 \mathrm{~B}-\mathrm{C} 20 \mathrm{~B}-\mathrm{C} 19 \mathrm{~B}$ & $178.1(4)$ \\
\hline $\mathrm{C} 8 \mathrm{~B}-\mathrm{C} 9 \mathrm{~B}-\mathrm{C} 10 \mathrm{~B}-\mathrm{C} 11 \mathrm{~B}$ & $-0.7(8)$ \\
\hline $\mathrm{C} 8 \mathrm{~B}-\mathrm{C} 13 \mathrm{~B}-\mathrm{C} 14 \mathrm{~B}-\mathrm{C} 15 \mathrm{~B}$ & $-0.1(7)$ \\
\hline $\mathrm{C} 8 \mathrm{C}-\mathrm{C} 7 \mathrm{C}-\mathrm{C} 20 \mathrm{C}-\mathrm{C} 15 \mathrm{C}$ & $-2.3(6)$ \\
\hline $\mathrm{C} 8 \mathrm{C}-\mathrm{C} 7 \mathrm{C}-\mathrm{C} 20 \mathrm{C}-\mathrm{C} 19 \mathrm{C}$ & $177.4(4)$ \\
\hline $\mathrm{C} 8 \mathrm{C}-\mathrm{C} 9 \mathrm{C}-\mathrm{C} 10 \mathrm{C}-\mathrm{C} 11 \mathrm{C}$ & $0.7(10)$ \\
\hline $\mathrm{C} 8 \mathrm{C}-\mathrm{C} 13 \mathrm{C}-\mathrm{C} 14 \mathrm{C}-\mathrm{C} 15 \mathrm{C}$ & $-0.7(8)$ \\
\hline $\mathrm{C} 8 \mathrm{D}-\mathrm{C} 7 \mathrm{D}-\mathrm{C} 20 \mathrm{D}-\mathrm{C} 15 \mathrm{D}$ & $-2.5(7)$ \\
\hline $\mathrm{C} 8 \mathrm{D}-\mathrm{C} 7 \mathrm{D}-\mathrm{C} 20 \mathrm{D}-\mathrm{C} 19 \mathrm{D}$ & $176.5(4)$ \\
\hline $\mathrm{C} 8 \mathrm{D}-\mathrm{C} 9 \mathrm{D}-\mathrm{C} 10 \mathrm{D}-\mathrm{C} 11 \mathrm{D}$ & $-0.8(9)$ \\
\hline $\mathrm{C} 8 \mathrm{D}-\mathrm{C} 13 \mathrm{D}-\mathrm{C} 14 \mathrm{D}-\mathrm{C} 15 \mathrm{D}$ & $-1.8(8)$ \\
\hline $\mathrm{C} 9 \mathrm{~A}-\mathrm{C} 8 \mathrm{~A}-\mathrm{C} 13 \mathrm{~A}-\mathrm{C} 12 \mathrm{~A}$ & $-1.0(7)$ \\
\hline $\mathrm{C} 9 \mathrm{~A}-\mathrm{C} 8 \mathrm{~A}-\mathrm{C} 13 \mathrm{~A}-\mathrm{C} 14 \mathrm{~A}$ & $179.7(4)$ \\
\hline $\mathrm{C} 9 \mathrm{~A}-\mathrm{C} 10 \mathrm{~A}-\mathrm{C} 11 \mathrm{~A}-\mathrm{C} 12 \mathrm{~A}$ & $-2.1(9)$ \\
\hline $\mathrm{C} 9 \mathrm{~B}-\mathrm{C} 8 \mathrm{~B}-\mathrm{C} 13 \mathrm{~B}-\mathrm{C} 12 \mathrm{~B}$ & $-0.4(7)$ \\
\hline $\mathrm{C} 9 \mathrm{~B}-\mathrm{C} 8 \mathrm{~B}-\mathrm{C} 13 \mathrm{~B}-\mathrm{C} 14 \mathrm{~B}$ & $-179.0(5)$ \\
\hline $\mathrm{C} 9 \mathrm{~B}-\mathrm{C} 10 \mathrm{~B}-\mathrm{C} 11 \mathrm{~B}-\mathrm{C} 12 \mathrm{~B}$ & $0.2(9)$ \\
\hline $\mathrm{C} 9 \mathrm{C}-\mathrm{C} 8 \mathrm{C}-\mathrm{C} 13 \mathrm{C}-\mathrm{C} 12 \mathrm{C}$ & $0.2(7)$ \\
\hline $\mathrm{C} 9 \mathrm{C}-\mathrm{C} 8 \mathrm{C}-\mathrm{C} 13 \mathrm{C}-\mathrm{C} 14 \mathrm{C}$ & $178.2(5)$ \\
\hline $\mathrm{C} 9 \mathrm{C}-\mathrm{C} 10 \mathrm{C}-\mathrm{C} 11 \mathrm{C}-\mathrm{C} 12 \mathrm{C}$ & $-0.1(11)$ \\
\hline $\mathrm{C} 9 \mathrm{D}-\mathrm{C} 8 \mathrm{D}-\mathrm{C} 13 \mathrm{D}-\mathrm{C} 12 \mathrm{D}$ & $1.0(7)$ \\
\hline $\mathrm{C} 9 \mathrm{D}-\mathrm{C} 8 \mathrm{D}-\mathrm{C} 13 \mathrm{D}-\mathrm{C} 14 \mathrm{D}$ & $-177.0(5)$ \\
\hline $\mathrm{C} 9 \mathrm{D}-\mathrm{C} 10 \mathrm{D}-\mathrm{C} 11 \mathrm{D}-\mathrm{C} 12 \mathrm{D}$ & $2.6(10)$ \\
\hline $\mathrm{C} 10 \mathrm{~A}-\mathrm{C} 11 \mathrm{~A}-\mathrm{C} 12 \mathrm{~A}-\mathrm{C} 13 \mathrm{~A}$ & $1.1(10)$ \\
\hline $\mathrm{C} 10 \mathrm{~B}-\mathrm{C} 11 \mathrm{~B}-\mathrm{C} 12 \mathrm{~B}-\mathrm{C} 13 \mathrm{~B}$ & $0.2(9)$ \\
\hline $\mathrm{C} 10 \mathrm{C}-\mathrm{C} 11 \mathrm{C}-\mathrm{C} 12 \mathrm{C}-\mathrm{C} 13 \mathrm{C}$ & $-0.5(11)$ \\
\hline $\mathrm{C} 10 \mathrm{D}-\mathrm{C} 11 \mathrm{D}-\mathrm{C} 12 \mathrm{D}-\mathrm{C} 13 \mathrm{D}$ & $-2.6(10)$ \\
\hline $\mathrm{C} 11 \mathrm{~A}-\mathrm{C} 12 \mathrm{~A}-\mathrm{C} 13 \mathrm{~A}-\mathrm{C} 8 \mathrm{~A}$ & $0.5(9)$ \\
\hline $\mathrm{C} 11 \mathrm{~A}-\mathrm{C} 12 \mathrm{~A}-\mathrm{C} 13 \mathrm{~A}-\mathrm{C} 14 \mathrm{~A}$ & $179.7(6)$ \\
\hline $\mathrm{C} 11 \mathrm{~B}-\mathrm{C} 12 \mathrm{~B}-\mathrm{C} 13 \mathrm{~B}-\mathrm{C} 8 \mathrm{~B}$ & $-0.1(8)$ \\
\hline $\mathrm{C} 11 \mathrm{~B}-\mathrm{C} 12 \mathrm{~B}-\mathrm{C} 13 \mathrm{~B}-\mathrm{C} 14 \mathrm{~B}$ & $178.5(5)$ \\
\hline $\mathrm{C} 11 \mathrm{C}-\mathrm{C} 12 \mathrm{C}-\mathrm{C} 13 \mathrm{C}-\mathrm{C} 8 \mathrm{C}$ & $0.4(9)$ \\
\hline $\mathrm{C} 11 \mathrm{C}-\mathrm{C} 12 \mathrm{C}-\mathrm{C} 13 \mathrm{C}-\mathrm{C} 14 \mathrm{C}$ & $-177.6(6)$ \\
\hline $\mathrm{C} 11 \mathrm{D}-\mathrm{C} 12 \mathrm{D}-\mathrm{C} 13 \mathrm{D}-\mathrm{C} 8 \mathrm{D}$ & $0.7(8)$ \\
\hline $\mathrm{C} 11 \mathrm{D}-\mathrm{C} 12 \mathrm{D}-\mathrm{C} 13 \mathrm{D}-\mathrm{C} 14 \mathrm{D}$ & $178.6(6)$ \\
\hline $\mathrm{C} 12 \mathrm{~A}-\mathrm{C} 13 \mathrm{~A}-\mathrm{C} 14 \mathrm{~A}-\mathrm{C} 15 \mathrm{~A}$ & $-179.2(5)$ \\
\hline
\end{tabular}

$\mathrm{C} 6 \mathrm{~F}-\mathrm{C} 1 \mathrm{~F}-\mathrm{C} 2 \mathrm{~F}-\mathrm{C} 3 \mathrm{~F} \quad 0.0$

$\mathrm{C} 6 \mathrm{~F}-\mathrm{C} 7 \mathrm{~F}-\mathrm{C} 8 \mathrm{~F}-\mathrm{C} 9 \mathrm{~F} \quad 1.9(5)$

$\mathrm{C} 6 \mathrm{~F}-\mathrm{C} 7 \mathrm{~F}-\mathrm{C} 8 \mathrm{~F}-\mathrm{C} 13 \mathrm{~F} \quad-179.2(5)$

$\mathrm{C} 6 \mathrm{~F}-\mathrm{C} 7 \mathrm{~F}-\mathrm{C} 20 \mathrm{~F}-\mathrm{C} 15 \mathrm{~F} \quad-178.6(5)$

$\mathrm{C} 6 \mathrm{~F}-\mathrm{C} 7 \mathrm{~F}-\mathrm{C} 20 \mathrm{~F}-\mathrm{C} 19 \mathrm{~F} \quad 0.2(5)$

$\mathrm{C} 1 \mathrm{~F}-\mathrm{C} 6 \mathrm{~F}-\mathrm{C} 7 \mathrm{~F}-\mathrm{C} 8 \mathrm{~F} \quad-73.9(6)$

$\mathrm{C} 1 \mathrm{~F}-\mathrm{C} 6 \mathrm{~F}-\mathrm{C} 7 \mathrm{~F}-\mathrm{C} 20 \mathrm{~F} \quad 110.1(5)$

$\mathrm{C} 1 \mathrm{~F}-\mathrm{C} 2 \mathrm{~F}-\mathrm{C} 3 \mathrm{~F}-\mathrm{C} 4 \mathrm{~F} \quad 0.0$

$\mathrm{C} 2 \mathrm{~F}-\mathrm{C} 3 \mathrm{~F}-\mathrm{C} 4 \mathrm{~F}-\mathrm{C} 5 \mathrm{~F} \quad 0.0$

$\mathrm{C} 4 \mathrm{~F}-\mathrm{C} 5 \mathrm{~F}-\mathrm{C} 6 \mathrm{~F}-\mathrm{C} 1 \mathrm{~F} \quad 0.0$

$\mathrm{C} 4 \mathrm{~F}-\mathrm{C} 5 \mathrm{~F}-\mathrm{C} 6 \mathrm{~F}-\mathrm{C} 7 \mathrm{~F} \quad-176.9(7)$

$\mathrm{C} 7 \mathrm{~F}-\mathrm{C} 6 \mathrm{~F}-\mathrm{C} 1 \mathrm{~F}-\mathrm{O} 7 \quad 0.1(6)$

$\mathrm{C} 7 \mathrm{~F}-\mathrm{C} 6 \mathrm{~F}-\mathrm{C} 1 \mathrm{~F}-\mathrm{C} 2 \mathrm{~F} \quad 176.7$ (7)

$\mathrm{C} 7 \mathrm{~F}-\mathrm{C} 8 \mathrm{~F}-\mathrm{C} 9 \mathrm{~F}-\mathrm{C} 10 \mathrm{~F} \quad 179.6$

$\mathrm{C} 7 \mathrm{~F}-\mathrm{C} 8 \mathrm{~F}-\mathrm{C} 13 \mathrm{~F}-\mathrm{C} 12 \mathrm{~F} \quad-179.2$

$\mathrm{C} 7 \mathrm{~F}-\mathrm{C} 8 \mathrm{~F}-\mathrm{C} 13 \mathrm{~F}-\mathrm{C} 14 \mathrm{~F} \quad 0.8$

$\mathrm{C} 8 \mathrm{~F}-\mathrm{C} 7 \mathrm{~F}-\mathrm{C} 20 \mathrm{~F}-\mathrm{C} 15 \mathrm{~F} \quad 5.4$

$\mathrm{C} 8 \mathrm{~F}-\mathrm{C} 7 \mathrm{~F}-\mathrm{C} 20 \mathrm{~F}-\mathrm{C} 19 \mathrm{~F} \quad-175.8$

$\mathrm{C} 8 \mathrm{~F}-\mathrm{C} 9 \mathrm{~F}-\mathrm{C} 10 \mathrm{~F}-\mathrm{C} 11 \mathrm{~F} \quad 1.3$

$\mathrm{C} 8 \mathrm{~F}-\mathrm{C} 13 \mathrm{~F}-\mathrm{C} 14 \mathrm{~F}-\mathrm{C} 15 \mathrm{~F} \quad-0.9$

$\mathrm{C} 9 \mathrm{~F}-\mathrm{C} 8 \mathrm{~F}-\mathrm{C} 13 \mathrm{~F}-\mathrm{C} 12 \mathrm{~F} \quad-0.3$

$\mathrm{C} 9 \mathrm{~F}-\mathrm{C} 8 \mathrm{~F}-\mathrm{C} 13 \mathrm{~F}-\mathrm{C} 14 \mathrm{~F} \quad 179.8$

$\mathrm{C} 9 \mathrm{~F}-\mathrm{C} 10 \mathrm{~F}-\mathrm{C} 11 \mathrm{~F}-\mathrm{C} 12 \mathrm{~F} \quad-3.8$

$\mathrm{C} 10 \mathrm{~F}-\mathrm{C} 11 \mathrm{~F}-\mathrm{C} 12 \mathrm{~F}-\mathrm{C} 13 \mathrm{~F} \quad 4.3$

$\mathrm{C} 11 \mathrm{~F}-\mathrm{C} 12 \mathrm{~F}-\mathrm{C} 13 \mathrm{~F}-\mathrm{C} 8 \mathrm{~F} \quad-2.2$

$\mathrm{C} 11 \mathrm{~F}-\mathrm{C} 12 \mathrm{~F}-\mathrm{C} 13 \mathrm{~F}-\mathrm{C} 14 \mathrm{~F} \quad 177.7$

$\mathrm{C} 12 \mathrm{~F}-\mathrm{C} 13 \mathrm{~F}-\mathrm{C} 14 \mathrm{~F}-\mathrm{C} 15 \mathrm{~F} \quad 179.2$

$\mathrm{C} 13 \mathrm{~F}-\mathrm{C} 8 \mathrm{~F}-\mathrm{C} 9 \mathrm{~F}-\mathrm{C} 10 \mathrm{~F} \quad 0.7$

$\mathrm{C} 13 \mathrm{~F}-\mathrm{C} 14 \mathrm{~F}-\mathrm{C} 15 \mathrm{~F}-\mathrm{C} 16 \mathrm{~F} \quad-177.7$

$\mathrm{C} 13 \mathrm{~F}-\mathrm{C} 14 \mathrm{~F}-\mathrm{C} 15 \mathrm{~F}-\mathrm{C} 20 \mathrm{~F} \quad 3.2$

$\mathrm{C} 14 \mathrm{~F}-\mathrm{C} 15 \mathrm{~F}-\mathrm{C} 16 \mathrm{~F}-\mathrm{C} 17 \mathrm{~F} \quad-178.3$

$\mathrm{C} 14 \mathrm{~F}-\mathrm{C} 15 \mathrm{~F}-\mathrm{C} 20 \mathrm{~F}-\mathrm{C} 7 \mathrm{~F} \quad-5.5$

$\mathrm{C} 14 \mathrm{~F}-\mathrm{C} 15 \mathrm{~F}-\mathrm{C} 20 \mathrm{~F}-\mathrm{C} 19 \mathrm{~F} \quad 175.7$

$\mathrm{C} 15 \mathrm{~F}-\mathrm{C} 16 \mathrm{~F}-\mathrm{C} 17 \mathrm{~F}-\mathrm{C} 18 \mathrm{~F} \quad 1.9$

$\mathrm{C} 16 \mathrm{~F}-\mathrm{C} 15 \mathrm{~F}-\mathrm{C} 20 \mathrm{~F}-\mathrm{C} 7 \mathrm{~F} \quad 175.4$

$\mathrm{C} 16 \mathrm{~F}-\mathrm{C} 15 \mathrm{~F}-\mathrm{C} 20 \mathrm{~F}-\mathrm{C} 19 \mathrm{~F} \quad-3.4$

$\mathrm{C} 16 \mathrm{~F}-\mathrm{C} 17 \mathrm{~F}-\mathrm{C} 18 \mathrm{~F}-\mathrm{C} 19 \mathrm{~F} \quad-2.0$

$\mathrm{C} 17 \mathrm{~F}-\mathrm{C} 18 \mathrm{~F}-\mathrm{C} 19 \mathrm{~F}-\mathrm{C} 20 \mathrm{~F}-0.6$

$\mathrm{C} 18 \mathrm{~F}-\mathrm{C} 19 \mathrm{~F}-\mathrm{C} 20 \mathrm{~F}-\mathrm{C} 7 \mathrm{~F} \quad-175.5$

$\mathrm{C} 18 \mathrm{~F}-\mathrm{C} 19 \mathrm{~F}-\mathrm{C} 20 \mathrm{~F}-\mathrm{C} 15 \mathrm{~F} \quad 3.3$

$\mathrm{C} 20 \mathrm{~F}-\mathrm{C} 7 \mathrm{~F}-\mathrm{C} 8 \mathrm{~F}-\mathrm{C} 9 \mathrm{~F} \quad 178.0$

$\mathrm{C} 20 \mathrm{~F}-\mathrm{C} 7 \mathrm{~F}-\mathrm{C} 8 \mathrm{~F}-\mathrm{C} 13 \mathrm{~F} \quad-3.1$

$\mathrm{C} 20 \mathrm{~F}-\mathrm{C} 15 \mathrm{~F}-\mathrm{C} 16 \mathrm{~F}-\mathrm{C} 17 \mathrm{~F} \quad 0.8$

$\mathrm{C} 21 \mathrm{~B}-\mathrm{O} 6 \mathrm{~B}-\mathrm{C} 24 \mathrm{~B}-\mathrm{C} 23 \mathrm{~B} \quad-32.3(14)$

$\mathrm{C} 21 \mathrm{~B}-\mathrm{C} 22 \mathrm{~B}-\mathrm{C} 23 \mathrm{~B}-\mathrm{C} 24 \mathrm{~B} \quad 2.5(16)$

$\mathrm{C} 22 \mathrm{~B}-\mathrm{C} 23 \mathrm{~B}-\mathrm{C} 24 \mathrm{~B}-\mathrm{O} 6 \mathrm{~B} \quad 17.1$ (14)

$\mathrm{C} 24 \mathrm{~B}-\mathrm{O} 6 \mathrm{~B}-\mathrm{C} 21 \mathrm{~B}-\mathrm{C} 22 \mathrm{~B} \quad 32.6(16)$

$\mathrm{C} 29 \mathrm{~B}-\mathrm{C} 30 \mathrm{~B}-\mathrm{C} 31 \mathrm{~B}-\mathrm{C} 32 \mathrm{~B} \quad 175.6$ 


$\begin{array}{ll}\mathrm{C} 12 \mathrm{~B}-\mathrm{C} 13 \mathrm{~B}-\mathrm{C} 14 \mathrm{~B}-\mathrm{C} 15 \mathrm{~B} & -178.6(5) \\ \mathrm{C} 12 \mathrm{C}-\mathrm{C} 13 \mathrm{C}-\mathrm{C} 14 \mathrm{C}-\mathrm{C} 15 \mathrm{C} & 177.3(5) \\ \mathrm{C} 12 \mathrm{D}-\mathrm{C} 13 \mathrm{D}-\mathrm{C} 14 \mathrm{D}-\mathrm{C} 15 \mathrm{D} & -179.8(5) \\ \mathrm{C} 13 \mathrm{~A}-\mathrm{C} 8 \mathrm{~A}-\mathrm{C} 9 \mathrm{~A}-\mathrm{C} 10 \mathrm{~A} & 0.1(7) \\ \mathrm{C} 13 \mathrm{~A}-\mathrm{C} 14 \mathrm{~A}-\mathrm{C} 15 \mathrm{~A}-\mathrm{C} 16 \mathrm{~A} & -177.4(4) \\ \mathrm{C} 13 \mathrm{~A}-\mathrm{C} 14 \mathrm{~A}-\mathrm{C} 15 \mathrm{~A}-\mathrm{C} 20 \mathrm{~A} & 1.5(7) \\ \mathrm{C} 13 \mathrm{~B}-\mathrm{C} 8 \mathrm{~B}-\mathrm{C} 9 \mathrm{~B}-\mathrm{C} 10 \mathrm{~B} & 0.8(8) \\ \mathrm{C} 13 \mathrm{~B}-\mathrm{C} 14 \mathrm{~B}-\mathrm{C} 15 \mathrm{~B}-\mathrm{C} 16 \mathrm{~B} & -180.0(5) \\ \mathrm{C} 13 \mathrm{~B}-\mathrm{C} 14 \mathrm{~B}-\mathrm{C} 15 \mathrm{~B}-\mathrm{C} 20 \mathrm{~B} & -0.4(7) \\ \mathrm{C} 13 \mathrm{C}-\mathrm{C} 8 \mathrm{C}-\mathrm{C} 9 \mathrm{C}-\mathrm{C} 10 \mathrm{C} & -0.8(8) \\ \mathrm{C} 13 \mathrm{C}-\mathrm{C} 14 \mathrm{C}-\mathrm{C} 15 \mathrm{C}-\mathrm{C} 16 \mathrm{C} & -177.7(5) \\ \mathrm{C} 13 \mathrm{C}-\mathrm{C} 14 \mathrm{C}-\mathrm{C} 15 \mathrm{C}-\mathrm{C} 20 \mathrm{C} & -0.1(7) \\ \mathrm{C} 13 \mathrm{D}-\mathrm{C} 8 \mathrm{D}-\mathrm{C} 9 \mathrm{D}-\mathrm{C} 10 \mathrm{D} & -1.0(8) \\ \mathrm{C} 13 \mathrm{D}-\mathrm{C} 14 \mathrm{D}-\mathrm{C} 15 \mathrm{D}-\mathrm{C} 16 \mathrm{D} & 179.4(5) \\ \mathrm{C} 13 \mathrm{D}-\mathrm{C} 14 \mathrm{D}-\mathrm{C} 15 \mathrm{D}-\mathrm{C} 20 \mathrm{D} & -1.4(7) \\ \mathrm{C} 14 \mathrm{~A}-\mathrm{C} 15 \mathrm{~A}-\mathrm{C} 16 \mathrm{~A}-\mathrm{C} 17 \mathrm{~A} & -178.8(5) \\ \mathrm{C} 14 \mathrm{~A}-\mathrm{C} 15 \mathrm{~A}-\mathrm{C} 20 \mathrm{~A}-\mathrm{C} 7 \mathrm{~A} & -3.6(6) \\ \mathrm{C} 14 \mathrm{~A}-\mathrm{C} 15 \mathrm{~A}-\mathrm{C} 20 \mathrm{~A}-\mathrm{C} 19 \mathrm{~A} & 177.0(4) \\ \mathrm{C} 14 \mathrm{~B}-\mathrm{C} 15 \mathrm{~B}-\mathrm{C} 16 \mathrm{~B}-\mathrm{C} 17 \mathrm{~B} & 179.7(5) \\ \mathrm{C} 14 \mathrm{~B}-\mathrm{C} 15 \mathrm{~B}-\mathrm{C} 20 \mathrm{~B}-\mathrm{C} 7 \mathrm{~B} & 0.8(7) \\ \mathrm{C} 14 \mathrm{~B}-\mathrm{C} 15 \mathrm{~B}-\mathrm{C} 20 \mathrm{~B}-\mathrm{C} 19 \mathrm{~B} & -178.1(4) \\ \mathrm{C} 14 \mathrm{C}-\mathrm{C} 15 \mathrm{C}-\mathrm{C} 16 \mathrm{C}-\mathrm{C} 17 \mathrm{C} & 176.5(5) \\ \mathrm{C} 14 \mathrm{C}-\mathrm{C} 15 \mathrm{C}-\mathrm{C} 20 \mathrm{C}-\mathrm{C} 7 \mathrm{C} & 1.6(7) \\ \mathrm{C} 14 \mathrm{C}-\mathrm{C} 15 \mathrm{C}-\mathrm{C} 20 \mathrm{C}-\mathrm{C} 19 \mathrm{C} & -178.1(4) \\ \mathrm{C} 14 \mathrm{D}-\mathrm{C} 15 \mathrm{D}-\mathrm{C} 16 \mathrm{D}-\mathrm{C} 17 \mathrm{D} & 177.7(5) \\ \mathrm{C} 14 \mathrm{D}-\mathrm{C} 15 \mathrm{D}-\mathrm{C} 20 \mathrm{D}-\mathrm{C} 7 \mathrm{D} & 3.6(7) \\ \mathrm{C} 14 \mathrm{D}-\mathrm{C} 15 \mathrm{D}-\mathrm{C} 20 \mathrm{D}-\mathrm{C} 19 \mathrm{D} & -175.5(4) \\ \mathrm{C} 15 \mathrm{~A}-\mathrm{C} 16 \mathrm{~A}-\mathrm{C} 17 \mathrm{~A}-\mathrm{C} 18 \mathrm{~A} & 0.9(7) \\ \mathrm{C} 15 \mathrm{~B}-\mathrm{C} 16 \mathrm{~B}-\mathrm{C} 17 \mathrm{~B}-\mathrm{C} 18 \mathrm{~B} & -1.6(8) \\ \mathrm{C} 15 \mathrm{C}-\mathrm{C} 16 \mathrm{C}-\mathrm{C} 17 \mathrm{C}-\mathrm{C} 18 \mathrm{C} & 1.0(8) \\ \mathrm{C} 15 \mathrm{D}-\mathrm{C} 16 \mathrm{D}-\mathrm{C} 17 \mathrm{D}-\mathrm{C} 18 \mathrm{D} & -1.9(8) \\ \mathrm{C} 16 \mathrm{~A}-\mathrm{C} 15 \mathrm{~A}-\mathrm{C} 20 \mathrm{~A}-\mathrm{C} 7 \mathrm{~A} & 175.3(4) \\ & \end{array}$

\begin{tabular}{|c|c|}
\hline $\mathrm{C} 29 \mathrm{~B}-\mathrm{C} 30 \mathrm{~B}-\mathrm{C} 35 \mathrm{~B}-\mathrm{C} 34 \mathrm{~B}$ & -175.4 \\
\hline $\mathrm{C} 30 \mathrm{~B}-\mathrm{C} 31 \mathrm{~B}-\mathrm{C} 32 \mathrm{~B}-\mathrm{C} 33 \mathrm{~B}$ & 0.0 \\
\hline $\mathrm{C} 31 \mathrm{~B}-\mathrm{C} 30 \mathrm{~B}-\mathrm{C} 35 \mathrm{~B}-\mathrm{C} 34 \mathrm{~B}$ & 0.3 \\
\hline $\mathrm{C} 31 \mathrm{~B}-\mathrm{C} 32 \mathrm{~B}-\mathrm{C} 33 \mathrm{~B}-\mathrm{C} 34 \mathrm{~B}$ & 0.0 \\
\hline $\mathrm{C} 32 \mathrm{~B}-\mathrm{C} 33 \mathrm{~B}-\mathrm{C} 34 \mathrm{~B}-\mathrm{C} 35 \mathrm{~B}$ & 0.1 \\
\hline $\mathrm{C} 33 \mathrm{~B}-\mathrm{C} 34 \mathrm{~B}-\mathrm{C} 35 \mathrm{~B}-\mathrm{C} 30 \mathrm{~B}$ & -0.2 \\
\hline $\mathrm{C} 35 \mathrm{~B}-\mathrm{C} 30 \mathrm{~B}-\mathrm{C} 31 \mathrm{~B}-\mathrm{C} 32 \mathrm{~B}$ & -0.2 \\
\hline $\mathrm{C} 43 \mathrm{~B}-\mathrm{C} 44 \mathrm{~B}-\mathrm{C} 45 \mathrm{~B}-\mathrm{C} 46 \mathrm{~B}$ & 175.6 \\
\hline $\mathrm{C} 43 \mathrm{~B}-\mathrm{C} 44 \mathrm{~B}-\mathrm{C} 49 \mathrm{~B}-\mathrm{C} 48 \mathrm{~B}$ & -175.4 \\
\hline $\mathrm{C} 44 \mathrm{~B}-\mathrm{C} 45 \mathrm{~B}-\mathrm{C} 46 \mathrm{~B}-\mathrm{C} 47 \mathrm{~B}$ & 0.0 \\
\hline $\mathrm{C} 45 \mathrm{~B}-\mathrm{C} 44 \mathrm{~B}-\mathrm{C} 49 \mathrm{~B}-\mathrm{C} 48 \mathrm{~B}$ & 0.2 \\
\hline $\mathrm{C} 45 \mathrm{~B}-\mathrm{C} 46 \mathrm{~B}-\mathrm{C} 47 \mathrm{~B}-\mathrm{C} 48 \mathrm{~B}$ & 0.0 \\
\hline $\mathrm{C} 46 \mathrm{~B}-\mathrm{C} 47 \mathrm{~B}-\mathrm{C} 48 \mathrm{~B}-\mathrm{C} 49 \mathrm{~B}$ & 0.1 \\
\hline $\mathrm{C} 47 \mathrm{~B}-\mathrm{C} 48 \mathrm{~B}-\mathrm{C} 49 \mathrm{~B}-\mathrm{C} 44 \mathrm{~B}$ & -0.2 \\
\hline $\mathrm{C} 49 \mathrm{~B}-\mathrm{C} 44 \mathrm{~B}-\mathrm{C} 45 \mathrm{~B}-\mathrm{C} 46 \mathrm{~B}$ & -0.1 \\
\hline $\mathrm{C} 36 \mathrm{~B}-\mathrm{C} 37 \mathrm{~B}-\mathrm{C} 38 \mathrm{~B}-\mathrm{C} 39 \mathrm{~B}$ & 175.6 \\
\hline $\mathrm{C} 36 \mathrm{~B}-\mathrm{C} 37 \mathrm{~B}-\mathrm{C} 42 \mathrm{~B}-\mathrm{C} 41 \mathrm{~B}$ & -175.5 \\
\hline $\mathrm{C} 37 \mathrm{~B}-\mathrm{C} 38 \mathrm{~B}-\mathrm{C} 39 \mathrm{~B}-\mathrm{C} 40 \mathrm{~B}$ & 0.0 \\
\hline $\mathrm{C} 38 \mathrm{~B}-\mathrm{C} 37 \mathrm{~B}-\mathrm{C} 42 \mathrm{~B}-\mathrm{C} 41 \mathrm{~B}$ & 0.3 \\
\hline $\mathrm{C} 38 \mathrm{~B}-\mathrm{C} 39 \mathrm{~B}-\mathrm{C} 40 \mathrm{~B}-\mathrm{C} 41 \mathrm{~B}$ & 0.0 \\
\hline $\mathrm{C} 39 \mathrm{~B}-\mathrm{C} 40 \mathrm{~B}-\mathrm{C} 41 \mathrm{~B}-\mathrm{C} 42 \mathrm{~B}$ & 0.0 \\
\hline $\mathrm{C} 40 \mathrm{~B}-\mathrm{C} 41 \mathrm{~B}-\mathrm{C} 42 \mathrm{~B}-\mathrm{C} 37 \mathrm{~B}$ & -0.2 \\
\hline $\mathrm{C} 42 \mathrm{~B}-\mathrm{C} 37 \mathrm{~B}-\mathrm{C} 38 \mathrm{~B}-\mathrm{C} 39 \mathrm{~B}$ & -0.2 \\
\hline $\mathrm{C} 36 \mathrm{~A}-\mathrm{C} 37 \mathrm{~A}-\mathrm{C} 38 \mathrm{~A}-\mathrm{C} 39 \mathrm{~A}$ & 175.6 \\
\hline $\mathrm{C} 36 \mathrm{~A}-\mathrm{C} 37 \mathrm{~A}-\mathrm{C} 42 \mathrm{~A}-\mathrm{C} 41 \mathrm{~A}$ & -175.5 \\
\hline $\mathrm{C} 37 \mathrm{~A}-\mathrm{C} 38 \mathrm{~A}-\mathrm{C} 39 \mathrm{~A}-\mathrm{C} 40 \mathrm{~A}$ & 0.0 \\
\hline $\mathrm{C} 38 \mathrm{~A}-\mathrm{C} 37 \mathrm{~A}-\mathrm{C} 42 \mathrm{~A}-\mathrm{C} 41 \mathrm{~A}$ & 0.2 \\
\hline $\mathrm{C} 38 \mathrm{~A}-\mathrm{C} 39 \mathrm{~A}-\mathrm{C} 40 \mathrm{~A}-\mathrm{C} 41 \mathrm{~A}$ & 0.1 \\
\hline $\mathrm{C} 39 \mathrm{~A}-\mathrm{C} 40 \mathrm{~A}-\mathrm{C} 41 \mathrm{~A}-\mathrm{C} 42 \mathrm{~A}$ & 0.0 \\
\hline $\mathrm{C} 40 \mathrm{~A}-\mathrm{C} 41 \mathrm{~A}-\mathrm{C} 42 \mathrm{~A}-\mathrm{C} 37 \mathrm{~A}$ & -0.1 \\
\hline $\mathrm{C} 42 \mathrm{~A}-\mathrm{C} 37 \mathrm{~A}-\mathrm{C} 38 \mathrm{~A}-\mathrm{C} 39 \mathrm{~A}$ & -0.2 \\
\hline
\end{tabular}

